\title{
Rwanda: Sixth Review Under the Three-Year Arrangement Under the Poverty Reduction and Growth Facility and Request for Waivers of Nonobservance of Performance Criteria-Staff Report; Press Release on the Executive Board Discussion; and Statement by the Executive Director for Rwanda
}

In the context of the sixth review under the three-year arrangement under the Poverty Reduction and Growth Facility and request for waivers of nonobservance of performance criteria with Rwanda, the following documents have been released and are included in this package:

- $\quad$ The staff report for the Sixth Review Under the Three-year Arrangement Under the Poverty Reduction and Growth Facility and Request for Waivers of Nonobservance of Performance criteria, prepared by a staff team of the IMF, following discussions that ended on July 7, 2009, with the officials of Rwanda on economic developments and policies. Based on information available at the time of these discussions, the staff report was completed on July 23, 2009. The views expressed in the staff report are those of the staff team and do not necessarily reflect the views of the Executive Board of the IMF.

- $\quad$ A Press Release summarizing the views of the Executive Board as expressed during its July 31, 2009 discussion of the staff report that completed the review and request.

- $\quad$ A statement by the Executive Director for Rwanda.

The documents listed below has [have] been or will be separately released.]

Letter of Intent sent to the IMF by the authorities of Rwanda* Memorandum of Economic and Financial Policies by the authorities of Rwanda*

*Also included in Staff Report

The policy of publication of staff reports and other documents allows for the deletion of market-sensitive information.

Copies of this report are available to the public from

International Monetary Fund • Publication Services $70019^{\text {th }}$ Street, N.W. • Washington, D.C. 20431

Telephone: (202) 623-7430 • Telefax: (202) 623-7201

E-mail: publications@imf.org •Internet: http://www.imf.org

\section{International Monetary Fund Washington, D.C.}





\section{INTERNATIONAL MONETARY FUND}

\section{RWANDA \\ Sixth Review Under the Three-Year Arrangement Under the Poverty Reduction and Growth Facility and Request for Waivers of Nonobservance of Performance Criteria}

Prepared by the African Department

(In collaboration with other departments)

Approved by Martine Guerguil and Dominique Desruelle

July 23, 2009

\section{Program Review}

- $\quad$ All end-2008 quantitative performance criteria were met and performance through March 2009 was broadly satisfactory.

- $\quad$ Staff recommends completion of the sixth review under the PRGF arrangement based on Rwanda's performance and understandings reached on the macroeconomic program for 2009/10.

- $\quad$ Staff supports the authorities' request for waivers of nonobservance of performance criteria on nonconcessional borrowing and nonintroduction of multiple currency practices.

\section{Participants}

- $\quad$ The mission team headed by Ms. Murgasova visited Kigali during May 18-29, 2009. The team comprised Mr. Mitchell, Mr. Gorbanyov, Mr. Roudet (all AFR) and Mr. Vandepeute (MCM) and was assisted by Mr. Gershenson, the resident representative. Mr. Bah, Senior Advisor to the Executive Director for Rwanda, and World Bank staff participated in the discussions. Ms. Murgasova and Mr. Mitchell visited Kigali again during June 22-July 7 to continue policy discussions.

- $\quad$ The mission met with Minister of Finance and Economic Planning Musoni, Governor Kanimba of the National Bank of Rwanda, other government officials and representatives of the private sector, civil society, and the international community. 
Executive Summary 3

I. Recent Developments and Program Performance .....................................................

II. Liquidity Issues in the Banking Sector ........................................................... 11

III. Macroeconomic Policies for 2009/10 and the Medium Term ......................................12

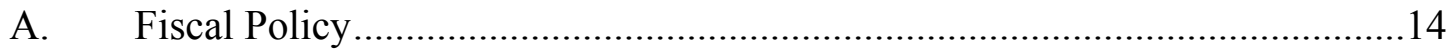

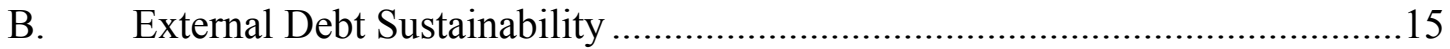

C. Monetary and Exchange Rate Policy.........................................................16

D. Addressing Credit Stagnation and Strengthening the Banking Sector ............17

IV. Performance Monitoring and Structural Reforms after the PRGF .................................18

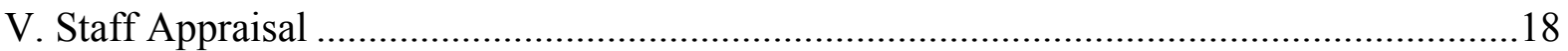

Tables

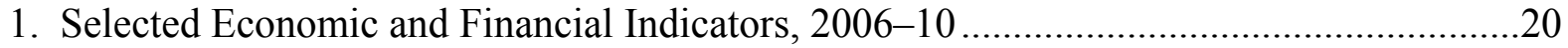

2a. Operations of the Central Government, Calendar Year Basis, 2006-09 .........................21

2b. Operations of the Central Government, Fiscal Year Basis, 2006/07-2011/12 _...............23

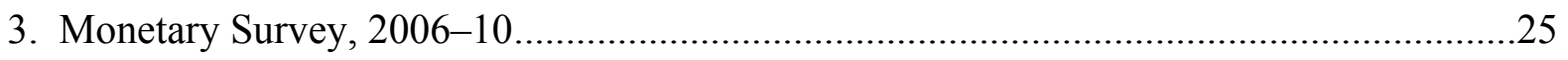

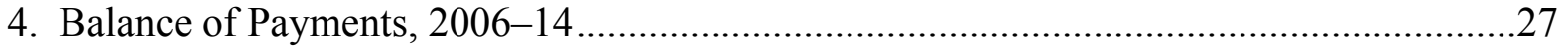

5. Financial Soundness Indicators for the Banking Sector, 2006-March 2009 .........28

6. Millenium Development Goals...........................................................................29

7. Proposed Schedule of Disbursements Under the PRGF Arrangement, 2009....................30

Figures

1. Rwanda: GDP Growth, Inflation and Exchange Rate, 2003-09 ....................................5

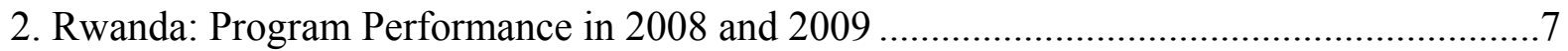

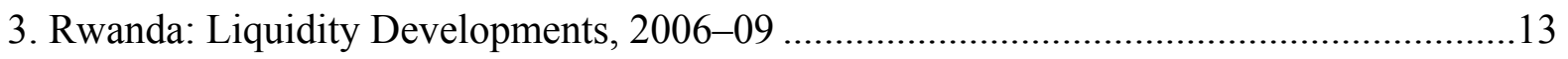

Boxes

1. Rwanda: Performance Under the PRGF-Supported Program .......................................10

2. Rwanda: Update of Selected DSA Indicators ............................................................... 16

Appendix

I. Letter of Intent

Attachment I: Memorandum of Economic and Financial Polices (MEFP) of the Government of Rwanda..................................... 33

Attachment I. Table 1: Quantitative Performance Criteria and Benchmarks for 2008....50 Attachment I. Table 2: Quantitative Indicative Targets and Benchmarks for 2009-10...51 


\section{EXECUTIVE SUMMARY}

Program performance was broadly satisfactory. Economic growth reached 11.2 percent in 2008, exceeding initial expectations. Inflation accelerated to above 20 percent in the second half of last year, but declined sharply in early 2009 because of falling world commodity prices, a favorable local harvest, and subsiding domestic demand pressures. All quantitative end-December 2008 performance criteria (PCs) were met. However, commitments to save all additional revenues and to increase interest rates were not observed. Liquidity pressures among banks emerged in late 2008 because of high credit growth and a decline in deposits.

In early 2009, external shocks affecting Rwanda created new challenges for macroeconomic policies. The impact of the global economic crisis led to a sharp fall in Rwandan export revenues and a slowdown of economic activity. Liquidity shortages exposed weaknesses in risk management and governance of commercial banks, prompting them to tighten credit standards and leading to a decline in credit growth. The involvement of the Rwandan military in resolving the conflict in the Eastern DRC necessitated an increase in fiscal spending. The situation was exacerbated by delays in donor disbursements, and the government drew on its deposits to finance expenditures. Several indicative targets for endMarch 2009 were therefore missed, including domestic fiscal balance, net credit to government, and net foreign assets.

The macroeconomic program for the 2009/10 fiscal year is designed to cushion the impact of the global economic crisis while preserving medium-term fiscal and external sustainability. The 2009/10 fiscal program accommodates a moderately higher budget deficit to stimulate the economy, financed by drawing down government deposits at the central bank. Fiscal consolidation is envisaged over the medium term, but priority spending will be safeguarded. The monetary program in 2009/10 will focus on keeping inflation in single digits while making room for liquidity support to the banking system if needed.

Staff supports the authorities' request for waivers for nonobservance of program performance criteria on (i) contracting new nonconcessional external debt and (ii) nonintroduction of multiple currency practices (MCPs). In late June 2009, the authorities contracted an external loan from the ExImBank of China on less concessional terms than allowed under the program. The World Bank has confirmed the project's viability. Staff analysis indicates that contracting the loan does not significantly affect debt sustainability. Rwanda's development partners have indicated that the loan would not hinder access to concessional financing. In June 2009, the central bank eliminated the foreign exchange hedge facility, the introduction of which in March 2009 gave rise to nonobservance of the continuous performance criterion on the nonintroduction of MCPs.

Macroeconomic risks have increased. Spillovers from the world crisis, liquidity shortages or solvency problems in the banking system may require additional policy adjustments.

Staff recommends conclusion of the sixth review under the PRGF arrangement. 


\section{Recent Developments And Program Performance}

\section{Rwanda was largely unaffected by the world economic slowdown in 2008 and} experienced its impact only from early 2009 (Table 1 and Figure 1). At 11.2 percent, GDP growth in 2008 surpassed earlier projections and reflected the robust expansion in agriculture, aided by the government's crop-intensification program (which includes the provision of fertilizer and seeds and the expansion of harvest storage facilities) and favorable weather conditions. Manufacturing, services and construction also registered strong growth; the latter due to the rapid private-sector credit increase and scaling up of the public investment program. In early 2009, however, exports declined sharply and economic activity slowed down, particularly in the construction, mining, and tourism sectors.

2. Inflation rose sharply in $\mathbf{2 0 0 8}$ but declined in 2009. The lagged impact of rapid domestic demand expansion and rising world food and fuel prices propelled consumer price inflation above 20 percent from mid-2008. In early 2009, however, a rapid decline in world commodity and domestic food prices led to a moderation of inflation to 12.7 percent in May.

\section{The fairly stable nominal exchange rate and high inflation led to a rapid real} appreciation of the Rwandan franc in late 2008. The NBR modified the mechanism for determining the reference rate at which it sells foreign exchange to commercial banks, which somewhat increased exchange rate flexibility in the second half of $2008 .{ }^{1}$ However, the nominal depreciation was still much lower than the inflation rate, and in real effective terms the Rwandan franc appreciated by 22 percent during 2008. In 2009, the sharp reduction in seasonally adjusted inflation contributed to a real depreciation of almost 5 percent (Figure 1).

\section{A widening current account deficit, resulting in part from a worsening in the} terms of trade, was covered comfortably by public and private inflows (Tables 1 and 4). Receipts from both traditional agricultural exports (coffee and tea) and mining exports increased significantly in 2008. However, they were more than offset by the rapidly rising imports boosted by economic growth, government investments, world commodity price increases, and real appreciation. Foreign direct investment (FDI), donor aid, and concessional public borrowing more than covered the current account deficit and led to the accumulation of international reserves to over 5 months of imports.

\footnotetext{
${ }^{1}$ From July 30, 2008, the intervention exchange rate was based on the weighted average of the rates (average reference rate or ARR) applied by banks in their transactions with their clients during the week. In November 2008 the mechanism was changed from the ARR to the NBR's selling rate which is fixed at a margin of 0.6 percent above the ARR. The exchange rate was also recalculated and applied daily instead of weekly.
} 
Figure 1. Rwanda: GDP Growth, Inflation and Exchange Rate, 2003-09

GDP growth reached double digits in 2008, fueled by exceptional agriculture performance....

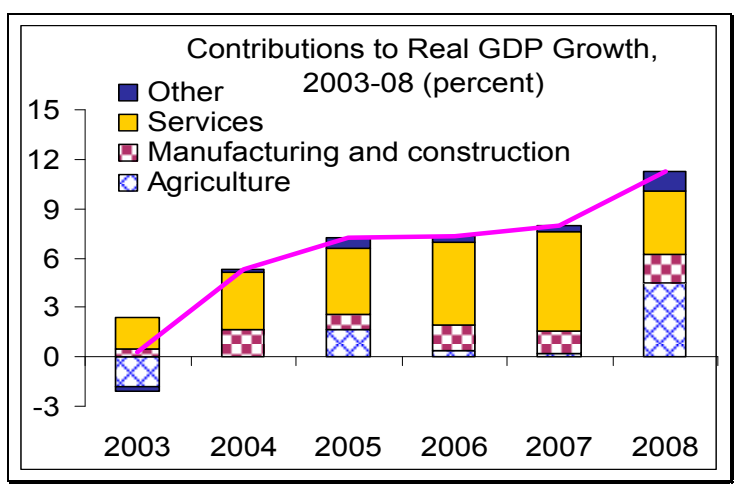

Inflation shot above 20 percent in 2008 but eased in early 2009...

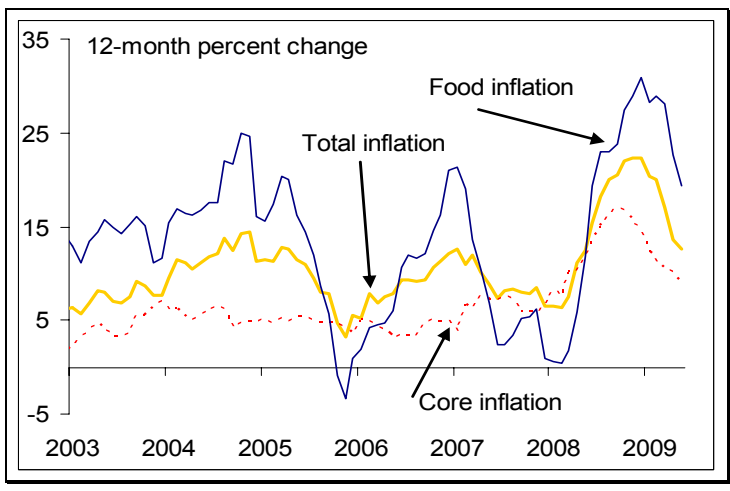

The real exchange rate appreciated significantly in the second half of $2008 . .$.

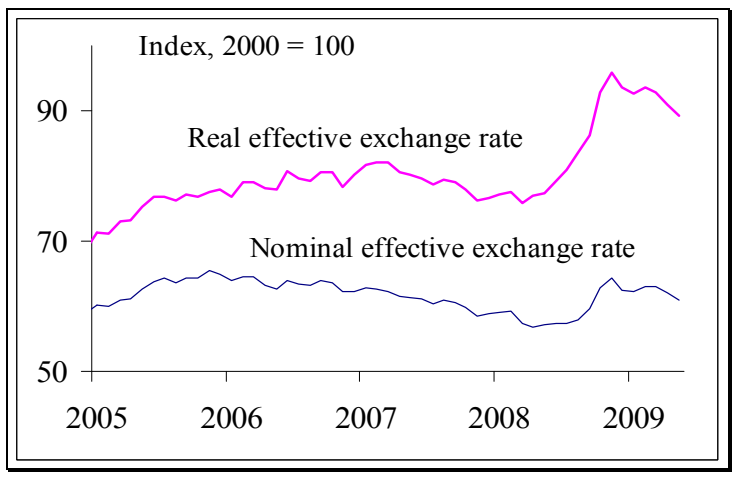

Source: Rwandan authorities; and staff estimates.
... and a construction boom, supported by public investment.

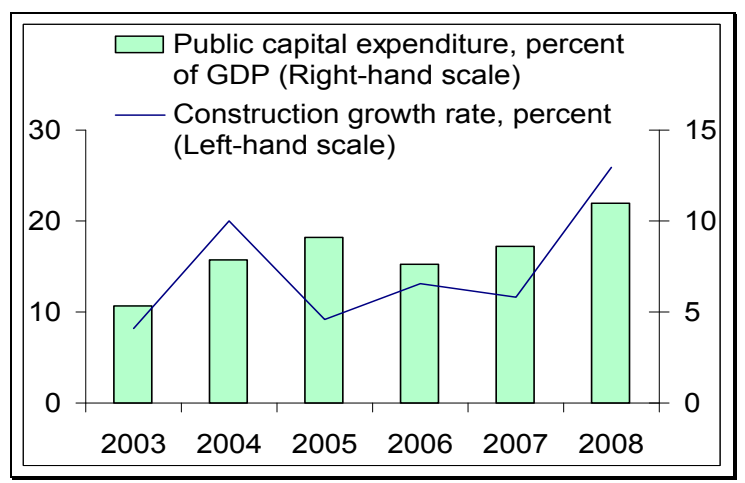

... amid increasing impact of domestic demand on prices.

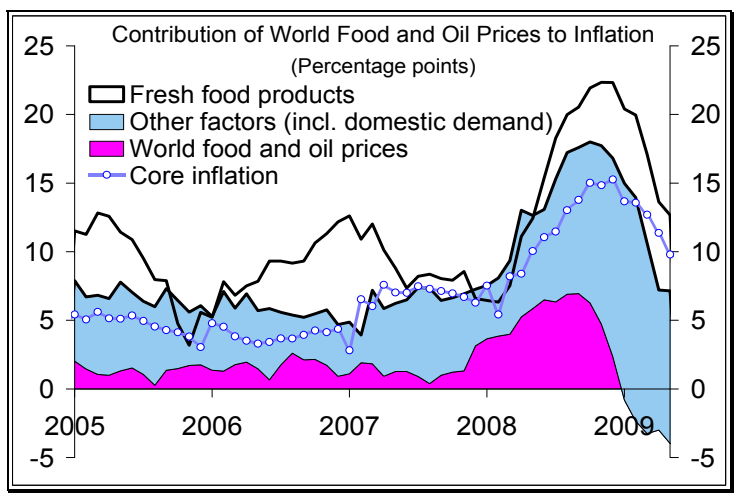

...while the nominal exchange rate slightly depreciated and stabilized thereafter.

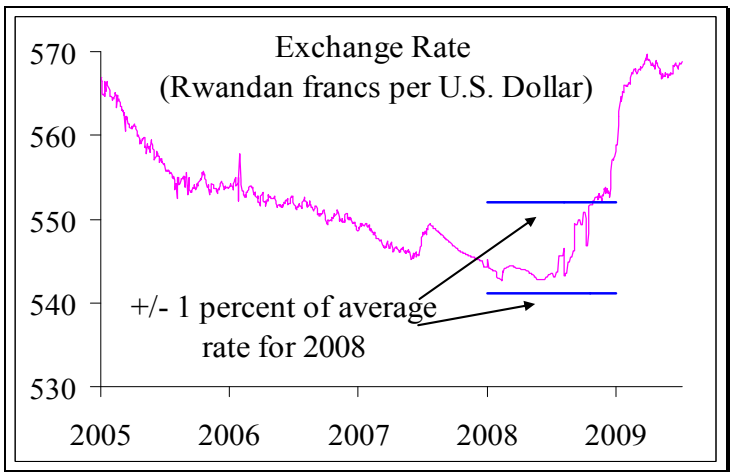


5. Rwanda's program performance through March 2009 was broadly satisfactory. All quantitative end-December 2008 performance criteria (PCs) for the sixth review were met. However, largely as a result of external and domestic shocks in early 2009 several indicative targets for end-March were missed (MEFP Tables 1 and 2, Figure 2). ${ }^{2}$

6. Fiscal performance in $\mathbf{2 0 0 8}$ was better than envisaged under the program, but delayed donor disbursements and external shocks created challenges to budget execution in early 2009 (Text Table 1, Table 2a).

- $\quad$ Revenues significantly exceeded program projections in 2008 , largely because of higher nominal GDP (boosted by economic growth and inflation) and unexpected one-off receipts. ${ }^{3}$ Despite the commitment in the last MEFP to save all extra tax revenues and ease the overheating pressures, part of the additional tax revenue was spent on capital projects that fueled domestic demand. Despite the economic slowdown, revenue collection during the first quarter of 2009 was broadly consistent with projections.

- The government accumulated large deposits in 2008, which provided a useful cushion against delayed donor disbursements in the first quarter of 2009. By saving unexpected non-tax revenues, the government accumulated deposits in the National Bank of Rwanda (NBR) of 1.8 percent of GDP in 2008. However, in the first quarter of 2009 the delayed disbursement of a large budget support grant from the World Bank (US\$79 million) led to a drawdown of government's deposits up to 1.5 percent of GDP. As a result, end-March indicative targets on the net credit to the government and the domestic fiscal balance were missed. Deposits were replenished once the delayed disbursement arrived in the second quarter.

- Budget spending exceeded projections. In 2008, current spending matched projections, but capital outlays and net lending significantly exceeded program targets. In early 2009, spending also surpassed projections, largely on account of unanticipated expenditure for military operations in the Eastern DRC (about 0.2 percent of GDP). The program targets on priority spending were met.

\footnotetext{
${ }^{2}$ The sixth review covers performance criteria for end-December 2008 and indicative targets for March 2009.

${ }^{3}$ The one-off receipts amounted to nearly 2 percent of GDP, and included telephone license fee of US\$60 million, arrears collection and revenues from ID cards distribution.
} 
Figure 2. Rwanda: Program Performance in 2008 and 2009

One-off receipts, higher growth and inflation pushed revenues above projections.

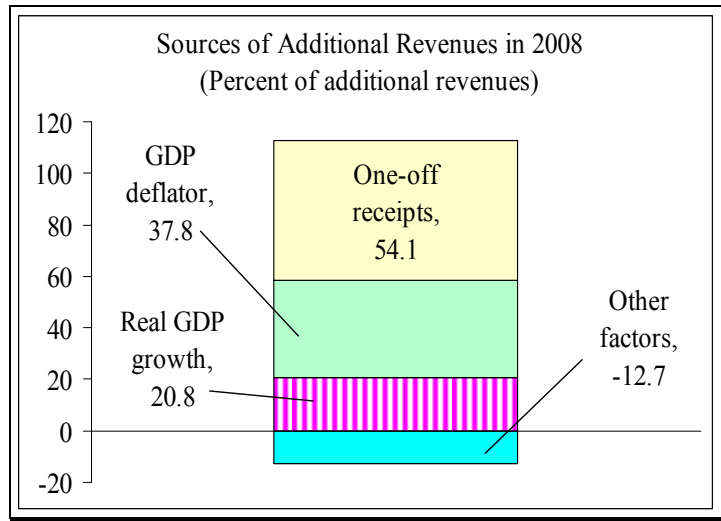

Intra-month levels of reserve money declined below projections in $2009 . .$.

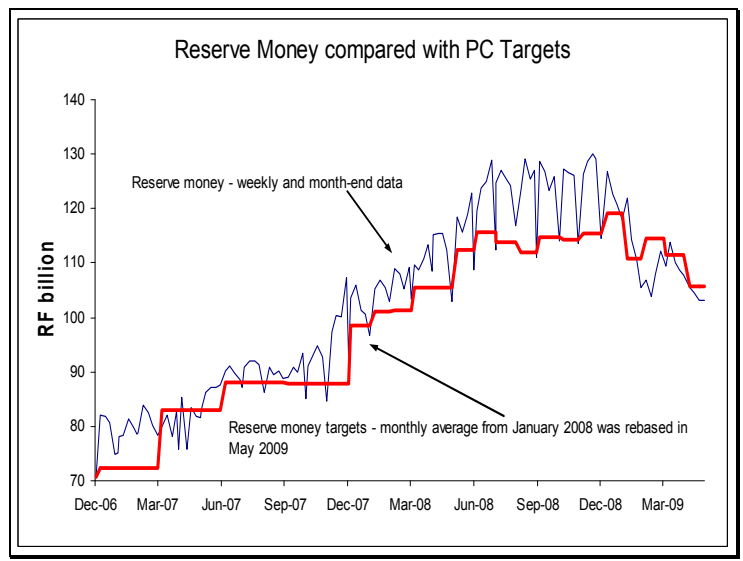

Source: Rwandan authorities; and staff estimates.
NFA met projections in 2008, but missed them in 2009, despite decline in FX sales.

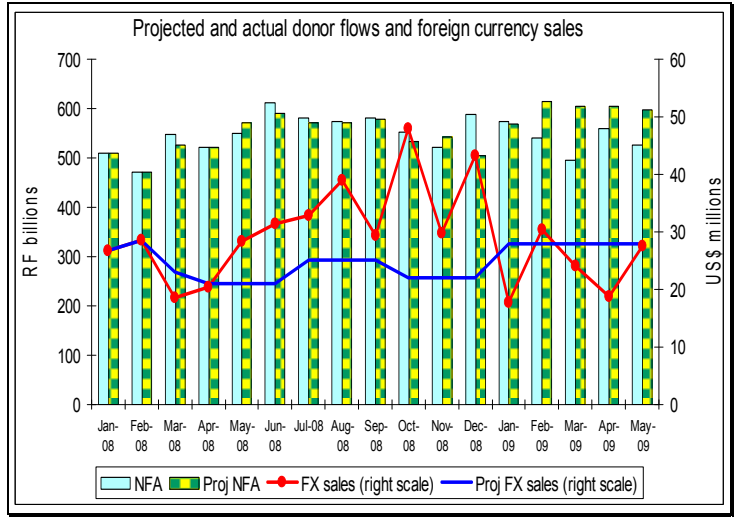

... and broad money also decreased well below projected path.

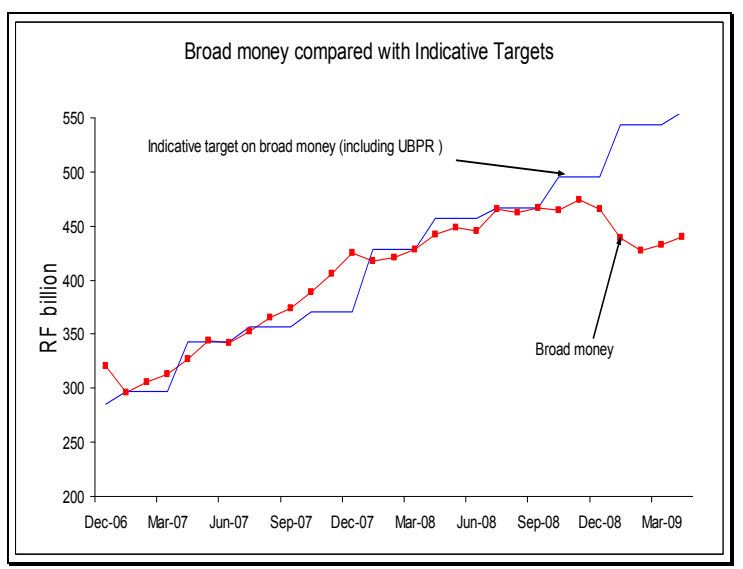

- $\quad$ The government accumulated new domestic payment arrears in early 2009. Having met the performance criterion for domestic arrears repayment in 2008, the indicative target for end-March was missed by RwF 5.6 billion ( 0.2 percent of GDP). This reflected administrative delays in processing transactions by the Treasury. According to preliminary information, the arrears were cleared by end-June 2009. 
Text Table 1. Rwanda: Operations of the Central Government, 2008-09

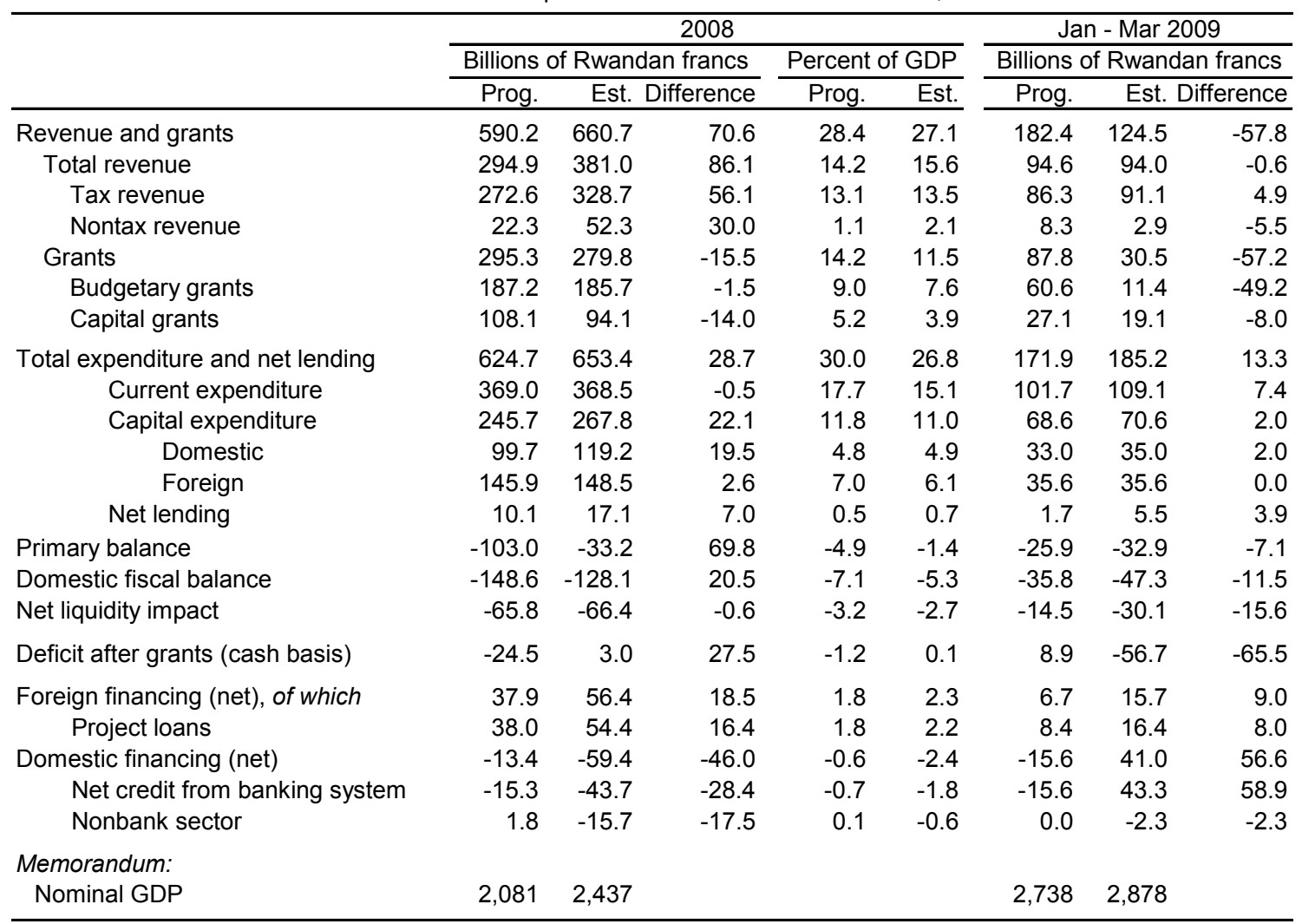

Sources: Rwandan authorities; and IMF staff estimates.

7. The monetary program remained broadly on track, but liquidity shortages in early $\mathbf{2 0 0 9}$ prompted the authorities to relax their monetary stance to curb the decline in broad money (Table 3 ).

Reserve money remained high on average during the second half of 2008. Despite meeting the end-month and quarterly targets, the intra-month levels of reserve money during the second half of 2008 increasingly exceeded the target path. This partially reflected the persistence of high levels of currency in circulation. The NBR relied primarily on foreign exchange sales for sterilization, but was not proactive in its use of other monetary instruments, including interest rate policy, to curb money demand despite prior commitments to do so. This sluggish response of monetary policy failed to curb the expansion of domestic demand in 2008 and inflationary pressures remained high.

- $\quad$ Reserve money and broad money decreased in nominal terms from late 2008.

Currency in circulation and deposits fell amid slowing economic activity, and consistent with the increased opportunity costs of holding balances in local currency in light of high inflation and negative real interest rates. In response, the authorities repaid a substantial amount of T-bills and reduced the reserve requirement from 8 percent to 5 percent, injecting large amounts of liquidity into the banking system 
(up to 1.5 percent of GDP). Broad money growth rebounded somewhat, but remains well below projections.

- The performance criterion for NFA was met at end-December, but the end-March indicative target was missed due to the slower-than-expected disbursement of grants. The situation improved in the second quarter, when some of the delayed disbursements were made.

8. Higher concessional borrowing led to faster accumulation of external debt. The present value of debt reached US\$331.5 million at end-2008, exceeding the program indicative target owing to frontloading of disbursements under existing loan contracts for externally financed projects.

\section{The authorities contracted a new nonconcessional loan from the China}

ExImBank at end-June 2009. The borrowing was foreshadowed in the MEFP for the fifth PRGF review. The loan (of about 0.6 percent of GDP) will finance a road improvement project in Kigali. The loan is on terms that are less concessional (about 34 percent grant element) than those allowed under the program (50 percent grant element). ${ }^{4}$

10. Structural PCs and benchmarks were not established for the sixth review.

\footnotetext{
${ }^{4}$ The loan has a maturity of 20 years (including a grace period of 6 years) and an annual interest rate of 2 percent. It also bears an upfront 1 percent management fee and a 0.3 percent commitment fee.
} 


\section{Box 1. Rwanda: Performance under the PRGF-Supported Program}

Sound economic and structural policies under the program contributed to economic growth, while inflation remained volatile. In 2006-08, the GDP growth averaged 8.8 percent, about twice the average growth rate of the three preceding years. However, average inflation exceeded the single-digit target, largely due to the effects of volatile international commodity and domestic food prices, and the lagged effects of rapid expansion of domestic demand.

\section{Resilience to external shocks}

improved. The low-access PRGF (10.68 percent of quota) helped mobilize highly concessional assistance from donors. In 2006-08, Rwanda received grants amounting to $10-11$ percent of GDP and contributing 40-45 percent of budget revenues. The high threshold for nonconcessional borrowing set in the PRGF (50 percent grant element) helped promote debt sustainability. In late 2008, Rwanda's rating of debt distress risk was revised from high to moderate. Accumulation of international reserves covering 5.3 months of

\begin{tabular}{|lrrr|}
\hline \multicolumn{3}{|c|}{ Rwanda: Selected Economic and Financial Indicators, $2006-08$} \\
\hline & 2006 & 2007 & 2008 \\
\hline Real GDP growth, percent & 7.3 & 7.9 & 11.2 \\
Inflation, percent (annual average) & 8.9 & 9.1 & 15.4 \\
Inflation, percent (end of year) & 12.2 & 6.6 & 22.3 \\
Central government budget (percent of GDP) & & & \\
Revenue & 13.3 & 13.6 & 15.6 \\
Grants & 10.7 & 9.9 & 11.5 \\
$\quad$ In percent of revenue and grants & 44.6 & 42.1 & 42.3 \\
Expenditure and net lending, of which & 24.5 & 24.9 & 26.7 \\
$\quad$ Current expenditure & 16.3 & 16.8 & 15.1 \\
Capital expenditure & 7.6 & 8.6 & 11.0 \\
Balance of payments (percent of GDP) & & & \\
Exports of goods and services & 12.3 & 12.2 & 14.9 \\
Imports of goods and services & 27.5 & 27.7 & 31.2 \\
Current account balance (incl. grants) & -4.7 & -2.4 & -5.5 \\
Gross official reservess (months of imports of G\&S) & 5.6 & 4.8 & 5.3 \\
Nominal GDP (billions of Rwandan francs) & 1564 & 1866 & 2437 \\
\hline Sources : Rwandan authorities; and staff estimates. & & & \\
\hline
\end{tabular}
imports created a cushion against balance of payments shocks.

The program supported structural reforms undertaken by the government. Reforms in tax administration widened the revenue base and helped raise revenues from 13.3 percent of GDP in 2006 to 15.6 percent of GDP in 2008. Improvements in PFM increased transparency and accountability of budget operations and boosted efficiency of spending. Financial sector reforms aim to modernize the payments system and improve access to credit and financial services, thus promoting credit growth and monetization of the economy. Reforms centered on improving the business climate contributed to private sector-led economic growth.

Yet despite strong economic growth, progress towards the MDGs was uneven. Rwanda is on track to achieving the MDGs in areas related to access to primary education, gender parity, malaria incidence and the prevalence of HIV. However, 56.9 percent of the population lives below the poverty line. Considerable efforts will be needed to meet the MDGs for reducing poverty and hunger, as well as for child and maternal mortality.

Going forward, policies should focus on building on the progress of recent years, further enhancing economic policy coordination and flexibility, and addressing capacity constraints in the public sector. In case of future rapid domestic demand expansion the policy response needs to be faster. Strengthening statistical data compilation, shortening the period of data production, improving data quality, and thus minimizing revisions of published data, would improve macroeconomic analysis. Expanding training would help alleviate the shortage of qualified local staff and improve the efficiency of public institutions. 


\section{LIQUidity ISSUES IN THE BANKING SECTOR}

11. The liquidity situation tightened from late 2008, raising concerns about bank stability and affecting credit growth (Figure 3). During 2008, negative real interest rates, increased competition in the banking sector, and more aggressive lending practices raised private sector credit growth to above 30 percent. After a period of rapid increase in 2007 and early 2008, deposits decelerated from mid-2008 and declined in nominal terms from November. As a result, bank liquidity tightened and the maturity mismatch between bank loans and deposits was exacerbated, thus reducing resources available for banks to provide credit to the economy. The credit expansion came to a halt from early 2009.

12. To facilitate payments and avoid credit stagnation, the authorities injected very large amounts of liquidity into the banking system in late 2008 and early 2009 ( $(77)$. This led to high aggregate excess reserves until the NBR resumed placing T-bills for sterilization purposes in May.

13. Credit growth stagnated despite abundant liquidity in the banking sector. While short-term liquidity conditions generally improved, many banks raised interest rates to attract time deposits and address maturity mismatches between their assets and liabilities, and have generally tightened lending standards, raising the risk of a prolonged credit slump with an adverse impact on the real economy.

\section{Two facilities introduced by the authorities in March to increase bank liquidity} and promote lending to the private sector were barely used, and in June the NBR eliminated one of them.

- $\quad$ A three to twelve month financing facility collateralized either by Treasury bonds or foreign currency deposits at an interest rate of 11.5 percent. Only one bank accessed the facility pledging Treasury bonds as collateral. Staff expressed concerns about the fixed pricing and the de jure unlimited nature of the scheme, which could potentially adversely affect the NBR's control over monetary aggregates. The authorities agreed to address these shortcomings (MEFP $\mid 40$ ).

- $\quad$ A foreign exchange risk hedge facility, through which banks could buy exchange rate insurance on their external borrowings for onlending to the economy. The facility was not self-financing because the annual fee of 3 percent to be paid by banks for the hedge was below the estimated price of an option providing similar rights as the scheme (calculated at 8.66 percent). This facility gave rise to an MCP subject to the Fund's jurisdiction under Article VIII, thus leading to non-observance of a continuous performance criterion under the PRGF. The NBR removed the facility on June 11, before it was used. On this basis, staff supports the authorities' request for a waiver of non-observance of the performance criterion. 
15. Asset quality and profitability indicators of banks have declined since mid-2008

(Table 5). As the asset quality of their portfolios deteriorated, banks have increased provisions against doubtful loans. A recent mission by the Monetary and Capital Markets Department found significant shortcomings in commercial banks' credit risk management and corporate governance, and weaknesses in bank supervision due to staffing shortages, which will be addressed (MEFP $q 44)$.

16. Responding to economic challenges and changes in regulation, several banks took steps to boost capital. In May, the NBR increased the capital adequacy requirement from 10 percent to 15 percent and modified the definition to include Tier 2 capital. While banks have 24 months to comply with the new regulation, it appears that most already meet it. Banks also reduced off-balance sheet items and insider loans, thus improving most measures of capital adequacy. Additionally, with the reduction in banks' foreign exchange exposures, market sensitivity to foreign exchange risks has been reduced (Table 5).

\section{MACroeconomic Policies for 2009/10 AND THE MEdiUm TeRM}

17. Three main factors are likely to influence economic performance in the next few years.

- Growth in the agricultural sector is expected to return to trend, following the exceptional performance in 2008. It could remain higher than average in 2009, reflecting a strong harvest so far this year, and is expected to return to its trend in 2010 .

- The global economic and financial crisis will take its toll on the Rwandan economy. The impact is expected to pass through three main channels: (i) declining external demand for Rwandan goods and services, particularly for tourism and mineral exports; (ii) slumping commodity prices, particularly for minerals; and (iii) scarcer financial inflows, including foreign direct investment (FDI). Despite early concerns, the aid flows are projected to remain high in 2009/10, while they are expected to decline over the medium-term. ${ }^{5}$

- $\quad$ The liquidity shortages in the banking sector could exacerbate the economic slowdown by reducing the availability of credit.

\footnotetext{
${ }^{5}$ While some donors are frontloading disbursements to mitigate the impact of the world economic crisis, medium-term projections include only grants already committed by donors.
} 
Figure 3. Rwanda: Liquidity Developments, 2006-09

\section{Declining real interest rates ...}

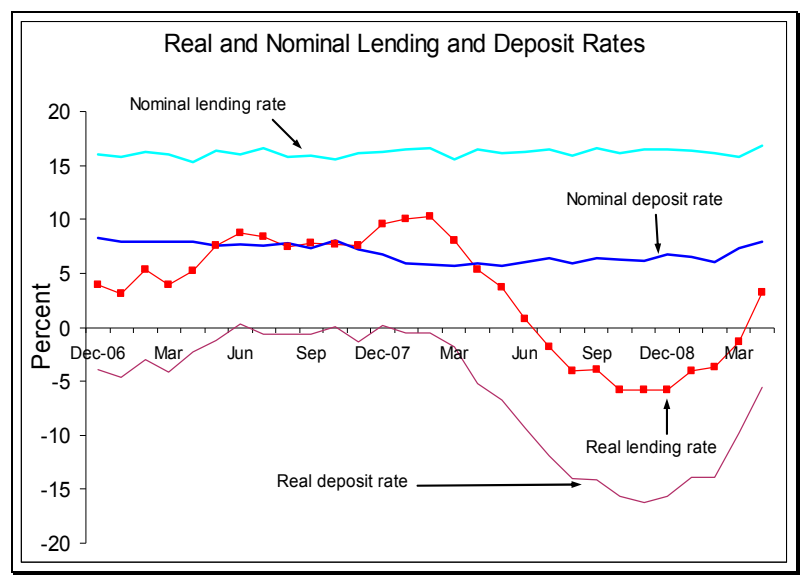

The structural liquidity surplus became a deficit.

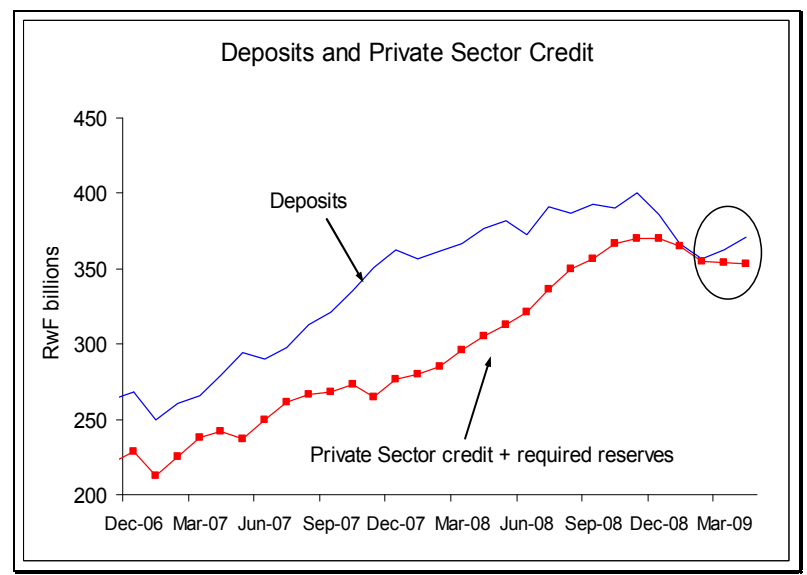

...lending by the NBR and the reduction in the reserve requirements.

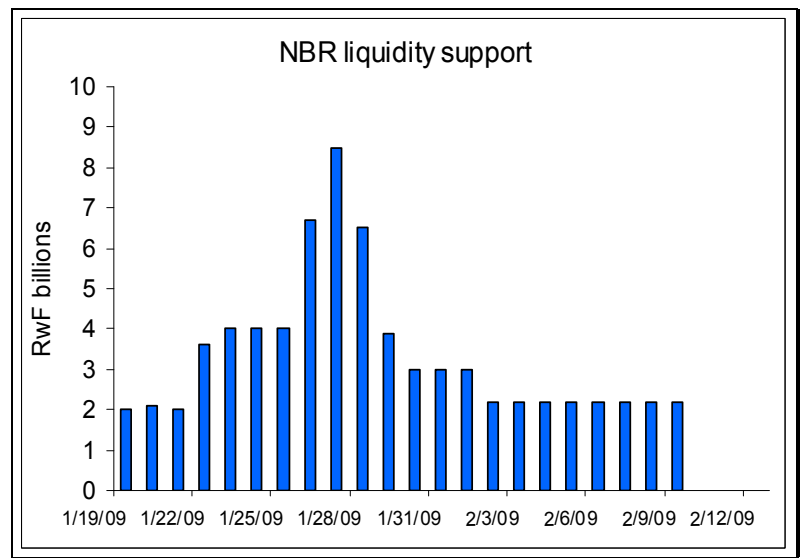

Source: Rwandan authorities; and staff estimates. ...contributed to accelerating credit growth and the decline in deposit growth.

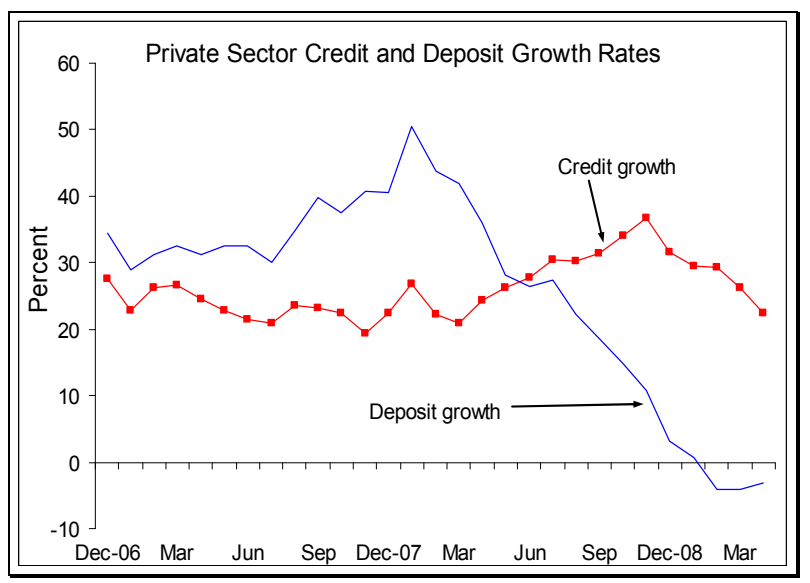

The policy response included repurchase of outstanding domestic assets...

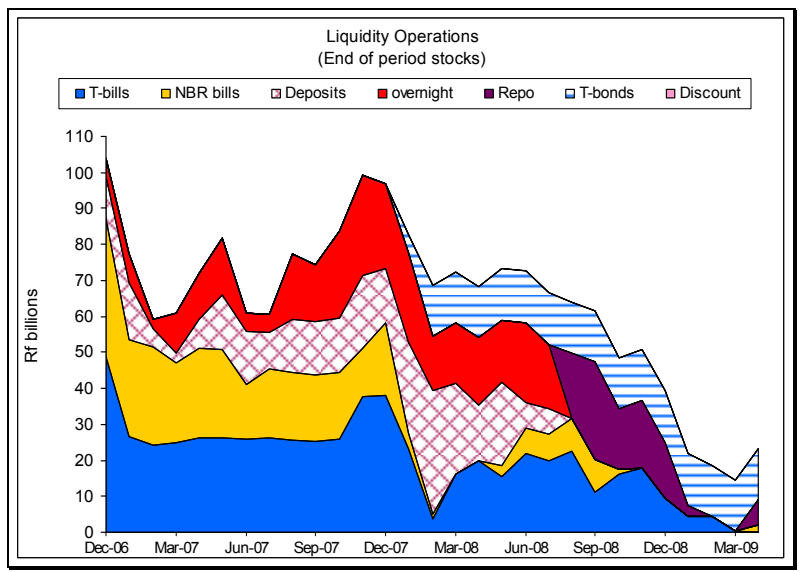

The liquidity situation improved and excess reserves have returned to prudential levels.

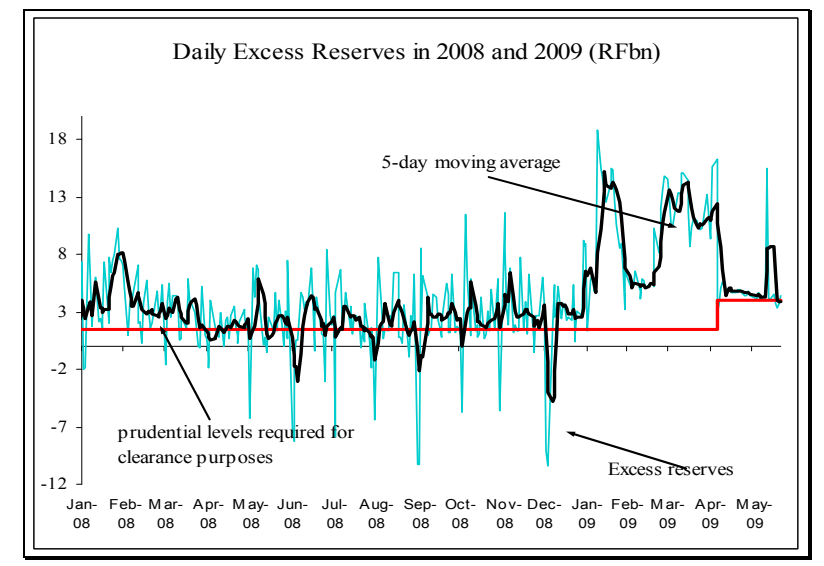


18. In light of these factors, the macroeconomic framework envisages the following:

- $\quad$ Economic growth is projected to slow to about 51/3 percent in 2009-10, with construction and real estate, mining, hotels and restaurants, and trade being particularly affected by the global economic crisis and the credit slowdown. While growth is projected to reach about 6 percent on average over the medium term, significant downside risks arise from the uncertain path of recovery in the world economy.

- Inflation is expected to decline to single digits in the second half of 2009 as a result of global disinflation, lower domestic food prices, and easing domestic demand pressures.

- $\quad$ The balance of payments is likely to deteriorate in 2009-10 before improving in the medium term, supported by the projected world recovery. In line with developments in the first quarter, exports of goods and services are projected to decrease significantly in 2009 - a decline that would be only partially offset by the projected reduction in imports. ${ }^{6}$ FDI and other capital inflows are also expected to fall, resulting in a deterioration of the overall balance of payment by about US $\$ 80$ million compared to 2008. In 2010, although exports of goods and services and private capital flows will likely start recovering, the projected increase in imports and decline in official transfers should lead to a further worsening of the current account.

- Some drawdown of international reserves can be accommodated over the next few years to cushion external shocks and avoid abrupt policy adjustments. The reserve coverage is projected to stabilize around 4 months of imports over the medium term - or $4 \frac{1}{2}$ months including the upcoming SDR allocation. Staff and the authorities agreed that the SDR allocation will be saved as a safeguard against potential balance of payments pressure in the medium term (MEFP 945 ).

19. In this context, macroeconomic policies will aim at cushioning the impact of the economic crisis on growth and poverty while preserving medium-term fiscal and external sustainability.

\section{A. Fiscal Policy}

20. The $2009 / 10$ fiscal program allows a moderate fiscal stimulus to minimize the adverse effects of the current economic slowdown (Table 2 b).

- $\quad$ Revenues are projected to fall by about $2 \frac{1}{2}$ percent of GDP compared to 2008/09, largely reflecting the absence of one-off receipts collected in 2008, slowing economic

\footnotetext{
${ }^{6}$ Private imports are adjusting with a considerable lag, while the government imports still remain high.
} 
activity, and losses from adopting the EAC common external tariff (CET). To partially offset the revenue losses, the government will introduce additional revenue measures of about 0.4 percent of GDP, which include increasing the fuel levy earmarked for road maintenance, reducing implicit subsidies for fuel products, and

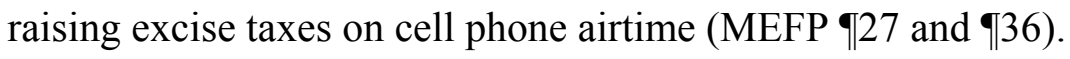

- While total spending will decline by about 1 percent of GDP compared to 2008/09, priority expenditures will remain broadly unchanged as a percent of GDP, consistent with the objectives outlined in Rwanda's Economic Development and Poverty Reduction Strategy (EDPRS) (MEFP q29).

- $\quad$ The expanding budget deficit in 2009/10 will be financed by withdrawing government deposits at the NBR up to 1 percent of GDP. This should provide a stimulus to the economy without rekindling undue inflation pressures or crowding out the private sector (MEFP $\{30$ ).

21. Gradual fiscal consolidation is envisaged over the medium term. Revenues are projected to increase by at least 0.2 percent of GDP per year, reflecting the economic recovery and the increasing efficiency of tax collection. Grant projections for 2010/11 and 2011/12 take into account only the amounts already committed by donors, resulting in a decline of 2-3 percent of GDP from the high level in 2009/10. To maintain current expenditures broadly unchanged as a share of GDP as in 2009/10, projections envisage an increase in concessional borrowing (which would be consistent with external debt sustainability), but no further recourse to domestic financing (MEFP q29).

\section{B. External Debt Sustainability}

\section{Staff supports the authorities' request for a waiver of non-observance of the} performance criterion (zero ceiling) on contracting new nonconcessional external debt from China ExImBank. More concessional financing for the project was not available. All requirements for staff support of the authorities' request for a waiver have been met: (i) the World Bank has confirmed the project's viability; (ii) staff's analysis suggests that the medium-term external debt sustainability has improved since the last DSA (produced in December 2008) because of an upward revision of export revenues, and the borrowing would not significantly worsen the debt outlook (Box 2); and (iii) development partners indicated that they do not view the contracting of the loan as an obstacle for Rwanda's access to concessional financing. Based on the above, staff believes that the contracting of the loan does not have implications on the authorities' ability to achieve the program objectives. 


\section{Box 2. Rwanda: Update of Selected DSA Indicators}

An upward revision of tourism travel receipts in the balance of payment statistics has led to a significant improvement in the debt sustainability analysis (DSA). The authorities have adopted a revised methodology for estimating travel receipts that has led to a marked upward revision of these estimates. As a result, the ratio of the PV of external public and publicly guaranteed (PPG) debt to exports of goods and services (traditionally the weakest DSA indicator for Rwanda) has improved considerably. While staff broadly agrees with the methodology used in estimating tourism receipts, there remain concerns - both from the authorities and staff-about a potential overestimation of tourism receipts. Staff therefore encourages the authorities to pursue their efforts to refine these estimates. The revised estimates suggest that Rwanda remains at a moderate risk of debt distress.

The contracting of nonconcessional loan from China ExImBank did not significantly worsen Rwanda's debt sustainability (see charts below).

Rwanda. External PPG Debt-to-Exports Ratio Under Alternative Scenarios

(All scenarios include the China ExImBank loan, unless otherwise noted)
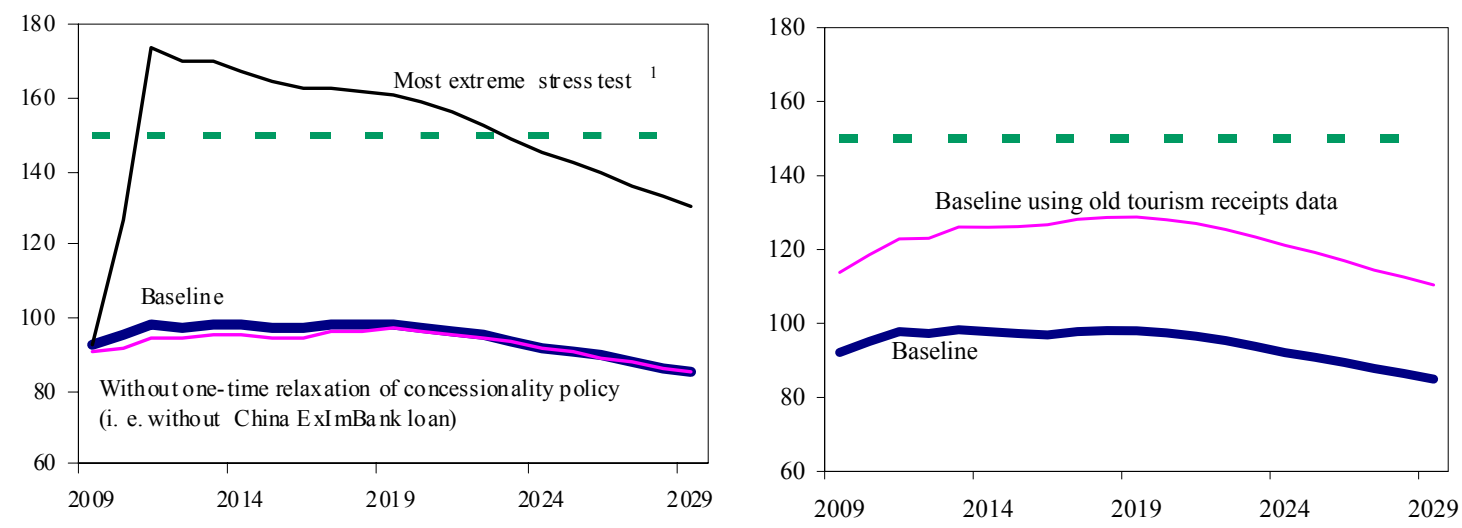

${ }^{1}$ Export value growth at historical average minus one standard deviation in 2010-11.

\section{Monetary and Exchange Rate Policy}

\section{The monetary program will aim to balance the objectives of reaching single-digit} inflation while providing sufficient liquidity to banking system. The agreed broad money targets for 2009/10 allow private sector credit growth of about 20 percent, consistent with the projected economic growth and single-digit inflation, and taking into account the envisaged external borrowing by commercial banks (Table 3 ).

\section{Reserve money will remain the anchor of the monetary program. Staff}

emphasized the need for the reserve money target to be met on average, not only at endmonth, through the coordinated use of available monetary policy instruments (sales of domestic securities and foreign currency). A more active use of interest rate policy supported by a proper communications strategy would help anchor interest rate expectations, reduce 
volatility, and strengthen the monetary policy transmission mechanism. The quantitative targets for reserve money were rebased to reflect the reduction of the reserve requirement and to accommodate a buffer of excess reserves ( $\mathrm{RwF} 4$ to 5 billion) that would support the efficient operations of the payments system and the inter-bank market for short-term liquidity.

25. Staff pointed out that greater exchange rate flexibility may be needed to stem the loss of foreign exchange reserves should the balance of payments deteriorate more than currently envisaged. Greater exchange rate flexibility may be required to counter the adverse effects of the global economic crisis and the impact of large real appreciation in 2008 that has affected external competitiveness. Developing a market based exchange rate, either by returning to the auction system or by moving swiftly toward an interbank foreign exchange market, ${ }^{7}$ would provide an excellent opportunity to foster such flexibility. The authorities broadly agreed with this approach (MEFP $\uparrow 38$ ).

26. Liquidity forecasting and coordination of fiscal and monetary policies need to be further improved. Staff encouraged the authorities to make liquidity management and monetary policy more effective by strengthening liquidity forecasting and improving coordination of fiscal and monetary policies. This would include enhanced monitoring of the domestic demand impact of fiscal spending and prompt adjustments in sterilization policies of the NBR to prevent shocks to domestic demand (MEFP $\mid 41$ ).

\section{Addressing Credit Stagnation and Strengthening the Banking Sector}

\section{Government intervention will be required to complement efforts of the} commercial banks to address the problem of maturity mismatches and the risks of prolonged credit stagnation. Staff welcomed efforts by some large commercial banks ${ }^{8}$ and the Rwanda Development Bank to directly borrow from international multilateral financial institutions for onlending to the private sector. It also recommended that the NBR establish a system to monitor external private sector debt. However, in light of the uncertainty regarding the availability of external financing for the banking sector, staff supports the authorities' proposal for a limited transfer of governments deposits from the NBR to commercial banks to encourage longer-term investment lending needed to sustain economic growth (MEFP 942). The scheme includes a number of safeguards, namely, commercial banks' voluntary participation, freedom to set the lending rates, their full assumption of credit risk, and exclusion of undercapitalized banks.

\footnotetext{
${ }^{7}$ Recommendations of the MCM TA mission to Rwanda (September 22- October 7, 2008).

${ }^{8}$ The larger banks have approached such institutions as FMO, EIB, IFC, and the ADB with requests for foreign currency loans.
} 
28. Staff recommended a number of measures to strengthen the health of the banking sector. These measures address liquidity and credit risks, corporate governance and risk management of commercial banks; as well as risk-based supervision and staffing of bank supervision (MEFP $₫ 44$ ).

\section{Performance Monitoring and Structural Reforms after the PRGF}

29. Staff discussed with the authorities Rwanda's relations with the Fund after the extended PRGF arrangement ends in August 2009. The authorities expressed an interest in a PSI from 2010 and to hold discussions on the PSI in early 2010 together with the consideration of the 2010/11 budget (MEFP $\mid 1$ ).

30. To facilitate the transition to a possible new program, staff and authorities agreed to set indicative targets and structural reform measures for the upcoming fiscal year. The authorities' MEFP outlines their macroeconomic policies for the 2009/10 (MEFP $\uparrow \uparrow 33-53$ ). After the PRGF arrangement expires, staff and the authorities will continue informal monitoring of the agreed targets.

31. Structural policies will continue to build on the broad based achievements of the past years. Focus will be on further reforms in the areas of public financial management, tax administration, financial sector development and improvement in the business environment.

\section{Staff Appraisal}

32. Rwanda's economic performance was broadly satisfactory in the second half of 2008. All program performance criteria and indicative targets were met, except for the indicative target on the present value of external debt, which was missed due to frontloading of disbursements for externally financed projects. Economic growth reached double digits, yet inflation increased to over 20 percent in the second half of 2008 due to rising world commodity prices and domestic demand expansion. Inflation pressures abated in early 2009 , and the government's objective to return inflation to single digits in the second half of this year is well within reach.

33. External and domestic shocks in early 2009 were challenging, and the policy response was broadly appropriate. Escalating conflict in the Eastern DRC temporarily worsened the security situation, and Rwanda's military involvement in its resolution necessitated additional budget spending. The world economic crisis began to affect Rwanda, and falling international prices and external demand caused a sharp contraction in the country's exports in the first quarter of the year. Even though imports are expected to adjust in the coming months, proactive policy actions may be needed to ensure balance of payments sustainability. While prompt actions by the NBR have largely resolved short-term liquidity shortages in the banking system, a limited transfer of government deposits from the central bank to commercial banks is appropriate to help prevent the prolonged stagnation of credit to the private sector. 
34. Staff recommended that the NBR's monetary and exchange rate policies need to become more proactive. The NBR should be ready to use exchange rate policy to ensure long-term sustainability of the balance of payments. Adherence to the agreed targets for reserve money consistent with single-digit inflation would provide a nominal anchor for monetary policy. Flexibility in the use of policy interest rates and strengthening the monetary policy framework will be essential for improving the monetary policy transmission mechanism and supporting financial deepening.

35. Staff urged the NBR to strengthen the functioning of the banking system and improve bank supervision. Weaknesses in risk management and corporate governance of commercial banks should be addressed. Additionally, the NBR should improve its staffing capacity and supervisory and monitoring tools.

36. Additional policy adjustments may be required should the world economic crisis and its impact on Rwanda worsen beyond current expectations or should the domestic banking situation deteriorate further. These may include actions to sustain external and budget sustainability, which can be affected by falling exports and diminishing fiscal revenues. In addition, the situation in the banking sector warrants close monitoring and prompt government support if liquidity shortages reoccur or solvency problems arise.

37. Staff welcomes the authorities' continued commitment to structural reforms. Staff encourages the authorities to persevere with the PFM and budget reforms, strengthening revenue administration and implementing the financial sector reform program. These efforts are also supported by Rwanda's developmental partners.

38. Staff supports the requested waivers for nonobservance of the program performance criteria on contracting new nonconcessional external debt and nonintroduction of MCPs and recommends completion of the sixth review under the PRGF arrangement. 
Table 1: Rwanda: Selected Economic and Financial Indicators, 2006-10

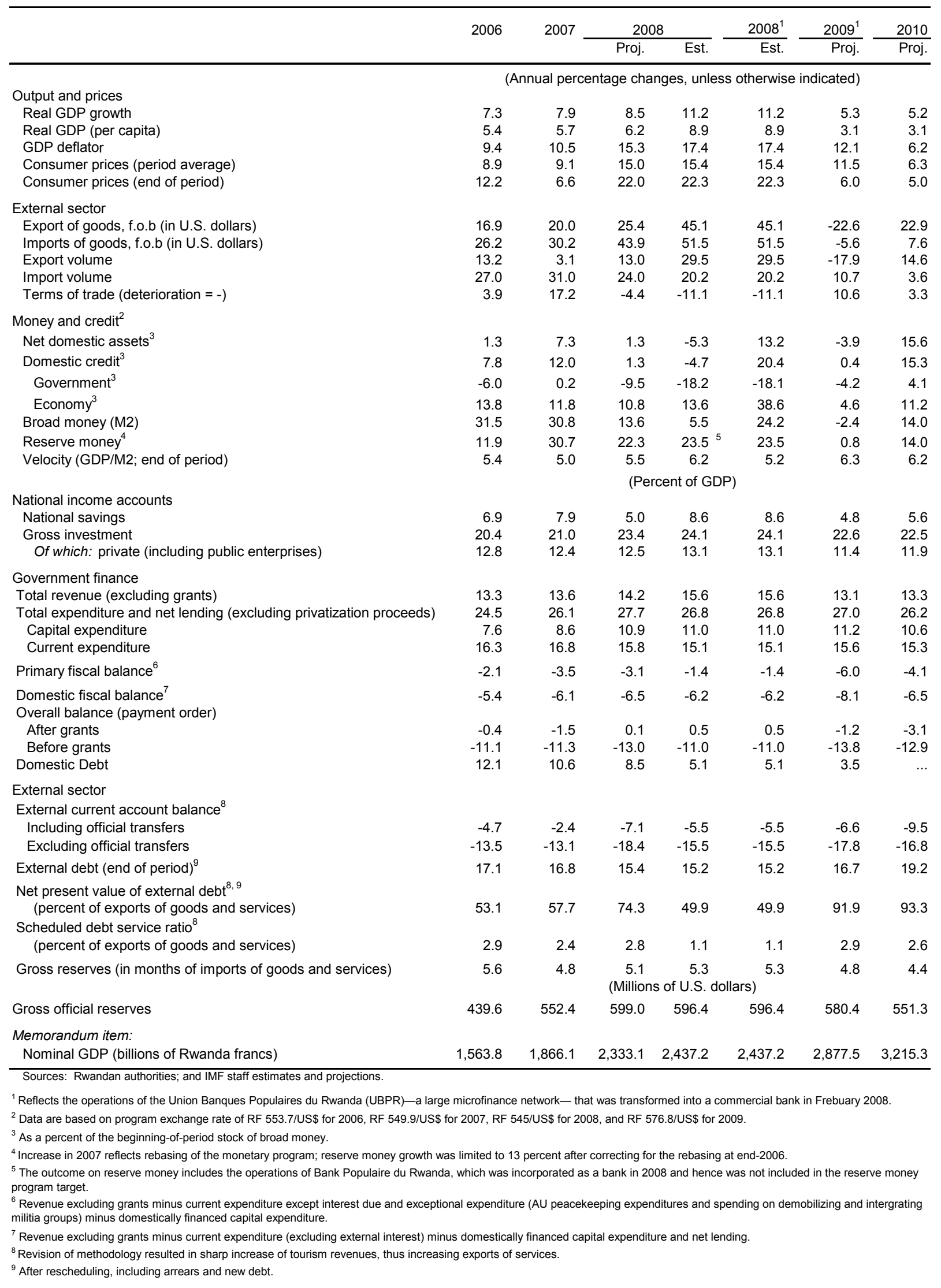


Table 2a. Rwanda: Operations of the Central Government, Calendar Year Basis, 2006-09

\begin{tabular}{|c|c|c|c|c|c|c|c|c|c|c|}
\hline & \multirow[t]{3}{*}{2006} & \multirow[t]{3}{*}{2007} & \multicolumn{4}{|c|}{2008} & \multicolumn{4}{|c|}{2009} \\
\hline & & & \multirow[t]{2}{*}{ June } & \multirow[t]{2}{*}{ Sep } & \multicolumn{2}{|c|}{ December } & \multicolumn{2}{|c|}{ March } & \multicolumn{2}{|c|}{ June } \\
\hline & & & & & Prog. & Est. & Prog. & Est. & Prog. & Proj. \\
\hline \multicolumn{11}{|c|}{ (Billions of Rwandan francs) } \\
\hline Revenue and grants & 376.0 & 436.8 & 318.8 & 448.6 & 590.2 & 660.7 & 182.4 & 124.5 & 363.6 & 354.0 \\
\hline Total revenue & 208.2 & 252.9 & 161.6 & 249.5 & 294.9 & 381.0 & 94.6 & 94.0 & 193.0 & 195.1 \\
\hline Direct taxes & 64.5 & 85.8 & 60.9 & 90.8 & 96.6 & 123.1 & 31.9 & 31.4 & 65.0 & 70.2 \\
\hline Taxes on goods and services & 95.5 & 121.0 & 71.4 & 114.4 & 142.0 & 161.7 & 42.8 & 46.2 & 87.2 & 88.5 \\
\hline Taxes on international trade & 33.6 & 31.0 & 18.6 & 29.8 & 34.0 & 43.9 & 11.7 & 13.6 & 23.8 & 19.4 \\
\hline Nontax revenue & 14.6 & 15.1 & 10.8 & 14.5 & 22.3 & 52.3 & 8.3 & 2.9 & 17.0 & 17.0 \\
\hline ID receipts & $\ldots$ & $\ldots$ & 0.6 & 1.8 & 5.5 & 3.2 & 0.7 & 0.7 & 1.5 & 1.5 \\
\hline Grants & 167.8 & 183.8 & 157.2 & 199.1 & 295.3 & 279.8 & 87.8 & 30.5 & 170.6 & 158.9 \\
\hline Budgetary grants & 71.9 & 133.3 & 122.3 & 143.6 & 187.2 & 185.7 & 60.6 & 11.4 & 115.2 & 127.4 \\
\hline Of which: HIPC Initiative assistance & 12.3 & 2.5 & 1.8 & 2.1 & 3.2 & 3.8 & 0.0 & 0.0 & 1.9 & 2.1 \\
\hline Project grants & 53.5 & 50.5 & 34.9 & 55.5 & 108.1 & 94.1 & 27.1 & 19.1 & 55.4 & 31.5 \\
\hline Total expenditure and net lending & 382.5 & 464.5 & 270.7 & 447.1 & 607.6 & 649.8 & 171.9 & 185.2 & 349.8 & 359.8 \\
\hline Total expenditure and net lending excluding privatization receipts & 382.5 & 486.6 & 274.3 & 450.7 & 624.7 & 653.4 & 171.9 & 185.2 & 349.8 & 359.8 \\
\hline Current expenditure & 254.1 & 312.6 & 173.9 & 275.3 & 369.0 & 368.5 & 101.7 & 109.1 & 206.5 & 220.6 \\
\hline Of which: priority & 127.7 & 161.0 & 89.8 & 145.3 & 193.0 & 204.8 & 50.4 & 57.8 & 103.0 & 103.0 \\
\hline Wages and salaries & 62.2 & 73.4 & 40.1 & 62.4 & 83.7 & 84.2 & 24.4 & 22.2 & 48.7 & 47.5 \\
\hline Civil & 41.2 & 53.7 & 28.6 & 45.1 & 60.1 & 61.0 & 17.7 & 15.6 & 35.3 & 34.1 \\
\hline Defense & 21.0 & 19.7 & 11.5 & 17.3 & 23.6 & 23.2 & 6.7 & 6.5 & 13.4 & 13.4 \\
\hline Purchases of goods and services & 71.6 & 77.5 & 35.1 & 56.9 & 78.2 & 80.3 & 24.7 & 27.9 & 50.5 & 62.3 \\
\hline Civil & 62.4 & 66.8 & 29.4 & 47.9 & 64.8 & 66.9 & 21.3 & 18.8 & 43.5 & 49.9 \\
\hline Defense & 9.1 & 10.7 & 5.8 & 9.0 & 13.4 & 13.4 & 3.4 & 9.1 & 7.0 & 12.4 \\
\hline Interest payments & 14.8 & 11.0 & 5.9 & 8.6 & 12.8 & 12.6 & 2.7 & 2.0 & 5.6 & 5.6 \\
\hline Domestic debt (due) & 8.6 & 8.7 & 3.9 & 6.2 & 9.6 & 8.9 & 2.0 & 1.3 & 4.0 & 3.5 \\
\hline External debt (due) & 6.2 & 2.3 & 1.9 & 2.4 & 3.2 & 3.8 & 0.8 & 0.7 & 1.6 & 2.1 \\
\hline Transfers & 71.9 & 103.8 & 64.2 & 100.7 & 136.2 & 130.4 & 38.4 & 41.9 & 78.4 & 81.8 \\
\hline Exceptional expenditure & 33.7 & 46.9 & 28.7 & 46.7 & 58.1 & 60.9 & 11.4 & 15.2 & 23.3 & 23.5 \\
\hline African Union peacekeeping & 10.9 & 11.0 & 5.3 & 9.8 & 10.0 & 15.9 & 3.9 & 6.6 & 8.0 & 9.1 \\
\hline Capital expenditure & 118.7 & 159.9 & 96.1 & 163.8 & 245.7 & 267.8 & 68.6 & 70.6 & 139.9 & 135.8 \\
\hline Of which: priority & 19.8 & 48.4 & 38.3 & 73.7 & 79.3 & 106.0 & 27.2 & 28.5 & 55.6 & 55.6 \\
\hline Domestic & 35.7 & 63.7 & 45.6 & 82.9 & 99.7 & 119.2 & 33.0 & 35.0 & 67.3 & 66.9 \\
\hline Foreign' & 83.0 & 96.2 & 50.5 & 80.9 & 145.9 & 148.5 & 35.6 & 35.6 & 72.6 & 68.9 \\
\hline Net lending and privatization receipts ${ }^{2}$ & 9.6 & -8.1 & 0.7 & 8.0 & -7.0 & 13.5 & 1.7 & 5.5 & 3.4 & 3.4 \\
\hline Of which: priority & 4.1 & 4.8 & 1.3 & 1.7 & 2.8 & 2.3 & 0.6 & 2.4 & 1.2 & 1.2 \\
\hline Of which: Rwandatel net receipts & -- & 22.1 & 3.6 & 3.6 & 13.6 & 3.6 & & & & \\
\hline Of which: BK receipts & -- & -- & -- & -- & 3.5 & 0.0 & & & & \\
\hline Primary balance ${ }^{3}$ & -33.2 & -65.5 & -23.4 & -53.5 & -103.0 & -33.2 & -25.9 & -32.9 & -51.9 & -63.4 \\
\hline Domestic fiscal balance $^{4}$ & -85.1 & -113.0 & -56.6 & -114.4 & -163.6 & -149.9 & -40.9 & -54.9 & -82.6 & -93.7 \\
\hline Excluding demobilization and peacekeeping expenditures ${ }^{5}$ & -70.8 & -98.2 & -48.9 & -100.1 & -148.6 & -128.1 & -35.8 & -47.3 & -72.2 & -81.1 \\
\hline Net liquidity impact ${ }^{6}$ & -13.4 & -62.5 & -28.3 & -28.3 & -67.7 & -50.7 & -14.5 & -27.8 & -22.2 & -26.0 \\
\hline Including nonbank domestic debt operations & -12.9 & -74.8 & -31.2 & -31.2 & -65.8 & -66.4 & -14.5 & -30.1 & -22.2 & -30.9 \\
\hline \multicolumn{11}{|l|}{ Overall deficit (payment order) } \\
\hline After grants & -6.5 & -27.7 & 48.1 & 1.5 & -17.5 & 11.0 & 10.5 & -60.6 & 13.8 & -5.8 \\
\hline Before grants & -174.3 & -211.6 & -109.0 & -197.7 & -312.8 & -268.8 & -77.3 & -91.2 & -156.8 & -164.7 \\
\hline Change in arrears ${ }^{7}$ & -7.5 & -8.5 & -4.0 & 4.6 & -7.0 & -8.0 & -1.6 & 4.0 & -3.3 & -3.3 \\
\hline Domestic & -7.5 & -8.5 & -4.0 & 4.6 & -7.0 & -8.0 & -1.6 & 4.0 & -3.3 & -3.3 \\
\hline External & 0.0 & 0.0 & 0.0 & 0.0 & 0.0 & 0.0 & 0.0 & 0.0 & 0.0 & 0.0 \\
\hline Deficit (-) & -14.0 & -36.2 & 44.1 & 6.1 & -24.5 & 3.0 & 8.9 & -56.7 & 10.5 & -9.1 \\
\hline
\end{tabular}


Table 2a. Rwanda: Operations of the Central Government, Calendar Year Basis, 2006-09 (concluded)

\begin{tabular}{|c|c|c|c|c|c|c|c|c|c|c|}
\hline & \multirow[t]{3}{*}{2006} & \multirow[t]{3}{*}{2007} & \multicolumn{4}{|c|}{2008} & \multicolumn{4}{|c|}{2009} \\
\hline & & & \multirow[t]{2}{*}{ June } & \multirow[t]{2}{*}{ Sep } & \multicolumn{2}{|c|}{ December } & \multicolumn{2}{|c|}{ March } & \multicolumn{2}{|c|}{ June } \\
\hline & & & & & Prog. & Est. & Prog. & Est. & Prog. & Proj. \\
\hline \multicolumn{11}{|c|}{ (Billions of Rwandan francs) } \\
\hline Financing & 15.2 & 36.2 & -44.2 & -6.1 & 24.5 & -3.0 & -8.8 & 56.7 & -10.5 & 9.1 \\
\hline Foreign financing (net) & 25.9 & 43.7 & 17.9 & 27.6 & 37.9 & 56.4 & 6.7 & 15.7 & 13.7 & 34.1 \\
\hline Drawings & 37.7 & 48.2 & 20.8 & 31.1 & 41.8 & 60.4 & 8.4 & 16.4 & 17.2 & 37.4 \\
\hline Budgetary loans & 8.2 & 0.6 & 5.2 & 5.8 & 3.8 & 5.9 & 0.0 & 0.0 & 0.0 & 0.0 \\
\hline Project loans & 29.5 & 47.7 & 15.6 & 25.3 & 38.0 & 54.4 & 8.4 & 16.4 & 17.2 & 37.4 \\
\hline Amortization & -11.8 & -4.5 & -2.9 & -3.5 & -3.9 & -4.0 & -1.7 & -0.8 & -3.5 & -3.3 \\
\hline Net domestic financing & -10.7 & -7.5 & -62.1 & -33.7 & -13.4 & -59.4 & -15.6 & 41.0 & -24.2 & -25.0 \\
\hline Net credit from banking system & -11.2 & 4.8 & -59.2 & -27.6 & -15.3 & -43.7 & -15.6 & 43.3 & -24.2 & -20.1 \\
\hline Nonbank sector (including CSR repayment) ${ }^{8}$ & 0.5 & -12.3 & -2.9 & -6.1 & 1.8 & -15.7 & 0.0 & -2.3 & 0.0 & -4.9 \\
\hline Errors and omissions / Financing gap ${ }^{9}$ & 1.2 & 0.0 & 0.0 & 0.0 & 0.0 & 0.0 & 0.0 & 0.0 & 0.0 & -0.1 \\
\hline \multicolumn{11}{|c|}{ (Percent of GDP, unless otherwise indicated) } \\
\hline Memorandum items: & & & & & & & & & & \\
\hline Revenue and grants & 24.0 & 23.4 & 13.1 & 18.4 & 28.4 & 27.1 & 6.7 & 4.3 & 13.3 & 12.3 \\
\hline Grants & 10.7 & 9.9 & 6.4 & 8.2 & 14.2 & 11.5 & 3.2 & 1.1 & 6.2 & 5.5 \\
\hline Revenue, excluding grants & 13.3 & 13.6 & 6.6 & 10.2 & 14.2 & 15.6 & 3.5 & 3.3 & 7.0 & 6.8 \\
\hline Revenue, excluding grants (percent of monetized sector GDP) & 21.2 & 20.8 & 10.5 & 16.1 & 22.3 & 24.7 & 7.4 & 5.3 & 11.3 & 10.9 \\
\hline Total expenditure and net lending & 24.5 & 24.9 & 11.1 & 18.3 & 29.2 & 26.7 & 6.3 & 6.4 & 12.8 & 12.5 \\
\hline Total expenditure and net lending excluding privatization receipts & 24.5 & 26.1 & 11.3 & 18.5 & 30.0 & 26.8 & 6.3 & 6.4 & 12.8 & 12.5 \\
\hline Of which: total priority expenditures & 9.7 & 11.5 & 5.3 & 9.1 & 13.2 & 12.8 & 2.9 & 3.1 & 5.8 & 5.6 \\
\hline Total expenditure & 23.8 & 25.3 & 11.1 & 18.0 & 29.5 & 26.1 & 6.2 & 6.2 & 12.7 & 12.4 \\
\hline Current expenditure & 16.3 & 16.8 & 7.1 & 11.3 & 17.7 & 15.1 & 3.7 & 3.8 & 7.5 & 7.7 \\
\hline Of which: wage bill & 4.0 & 3.9 & 1.6 & 1.7 & 4.0 & 3.5 & 0.9 & 0.8 & 1.8 & 1.6 \\
\hline goods and services & 4.6 & 4.2 & 1.4 & 1.6 & 3.8 & 3.3 & 0.9 & 1.0 & 1.8 & 2.2 \\
\hline Of which: defense/security & 1.9 & 1.6 & 0.7 & 0.7 & 1.8 & 1.5 & 0.4 & 0.5 & 0.7 & 0.9 \\
\hline exceptional expenditure & 2.2 & 2.5 & 1.2 & 0.9 & 2.8 & 2.5 & 0.4 & 0.5 & 0.9 & 0.8 \\
\hline Capital expenditure & 7.6 & 8.6 & 3.9 & 6.7 & 11.8 & 11.0 & 2.5 & 2.5 & 5.1 & 4.7 \\
\hline Domestic fiscal balance & -5.4 & -6.1 & -2.3 & -4.7 & -7.9 & -6.2 & -1.5 & -1.9 & -3.0 & -3.3 \\
\hline Excluding demobilization and peacekeeping expenditures ${ }^{5}$ & -4.5 & -5.3 & -2.0 & -4.1 & -7.1 & -5.3 & -1.3 & -1.6 & -2.6 & -2.8 \\
\hline Of which: domestic expenditure & 17.8 & 18.8 & 8.6 & 14.3 & 21.3 & 20.9 & 4.8 & 4.9 & 9.7 & 9.6 \\
\hline Net liquidity impact ${ }^{6}$ & -0.9 & -3.3 & -1.2 & -2.3 & -3.3 & -2.1 & -0.5 & -1.0 & -0.8 & -0.9 \\
\hline Including nonbank domestic debt operations & -0.8 & -4.0 & -1.3 & -2.5 & -3.2 & -2.7 & -0.5 & -1.0 & -0.8 & -1.1 \\
\hline Primary balance ${ }^{3}$ & -2.1 & -3.5 & -1.0 & -2.2 & -4.9 & -1.4 & -0.9 & -1.1 & -1.9 & -2.2 \\
\hline \multicolumn{11}{|l|}{ Overall deficit (payment order) } \\
\hline After grants & -0.4 & -1.5 & 2.0 & 0.1 & -0.8 & 0.5 & 0.4 & -2.1 & 0.5 & -0.2 \\
\hline Before grants & -11.1 & -11.3 & -4.5 & -8.1 & -15.0 & -11.0 & -2.8 & -3.2 & -5.7 & -5.7 \\
\hline Net domestic financing & -0.7 & -0.4 & -2.5 & -1.4 & -0.6 & -2.4 & -0.6 & 1.4 & -0.9 & -0.9 \\
\hline Net credit from banking system & -0.7 & 0.3 & -2.4 & -1.1 & -0.7 & -1.8 & -0.6 & 1.5 & -0.9 & -0.7 \\
\hline $\begin{array}{l}\text { Nominal GDP (RF billions) } \\
\text { Of which: monetized sectors }\end{array}$ & $\begin{array}{r}1,563.8 \\
9827\end{array}$ & $1,866.1$ & $2,437.2$ & $2,437.2$ & $2,081.1$ & $2,437.2$ & $2,737.6$ & $2,877.5$ & $2,737.6$ & $2,877.5$ \\
\hline Of which: monetized sectors & 982.7 & $1,216.5$ & $1,545.4$ & $1,545.4$ & $1,319.6$ & $1,545.4$ & $1,703.3$ & $1,790.3$ & $1,703.3$ & $1,790.3$ \\
\hline
\end{tabular}

Sources: Rwandan authorities, and IMF staff estimates and projections.

${ }^{1}$ For 2008, the increase partially reflects improvements in recording funds for projects.

2 Net lending in 2007 incorporates RF22.1 billion in receipts from the privatization of Rwandatel.

${ }^{3}$ Definition excludes exceptional expenditures, defined as total revenue (excluding privatization proceeds) minus

noninterest current expenditure (excluding exceptional expenditure) minus domestically financed capital expenditure.

${ }^{4}$ Revenue excluding grants minus current expenditure, domestically financed capital expenditure, and net lending,

In 2008, excludes RF 33.4 billion received for a telephone license fee.

${ }^{5}$ In 2006, peacekeeping activities of RF 2.9 billion were not covered by grants.

${ }^{6}$ Total expenditure and net lending (excluding privatization receipts) less revenues, foreign financed project spending,

and current spending on imports.

${ }^{7}$ A negative sign indicates a reduction.

${ }^{8}$ CSR = Caisse Sociale du Rwanda.

${ }^{9}$ A negative number implies underestimation of financing. 
Table 2b. Rwanda: Operations of the Central Government, Fiscal Year Basis ${ }^{1}$, 2006/07-2011/12

\begin{tabular}{|c|c|c|c|c|c|c|c|c|c|c|}
\hline & \multirow[t]{3}{*}{$2006 / 07$} & \multirow[t]{3}{*}{$2007 / 08$} & \multirow{2}{*}{\multicolumn{2}{|c|}{$\begin{array}{r}2008 / 09 \\
\text { Total }\end{array}$}} & \multicolumn{4}{|c|}{$2009 / 10$} & \multirow{3}{*}{$\begin{array}{r}2010 / 11 \\
\text { Proj. }\end{array}$} & \multirow{3}{*}{$\begin{array}{r}2011 / 12 \\
\text { Proj }\end{array}$} \\
\hline & & & & & \multirow{2}{*}{ Q3 2009} & Q4 2009 & Q1 2010 & \multirow{2}{*}{$\begin{array}{r}\text { Total } \\
\text { Proj. }\end{array}$} & & \\
\hline & & & Prog. & Proj. & & Projection & & & & \\
\hline \multicolumn{11}{|c|}{ (Billions of Rwandan francs) } \\
\hline Revenue and grants & 388.0 & 518.2 & 678.8 & 695.9 & 204.7 & 386.9 & 581.0 & 743.7 & 744.7 & 852.7 \\
\hline Total revenue & 233.2 & 290.3 & 362.0 & 414.4 & 90.1 & 183.0 & 296.1 & 401.5 & 455.3 & 520.9 \\
\hline Tax revenue & 217.6 & 272.4 & 334.8 & 355.9 & 82.8 & 168.0 & 272.0 & 368.0 & 417.7 & 478.7 \\
\hline Direct taxes & 73.6 & 103.3 & 118.4 & 132.3 & 31.4 & 63.8 & 107.1 & 147.1 & 168.4 & 194.0 \\
\hline Taxes on goods and services & 109.8 & 135.0 & 169.2 & 178.8 & 42.8 & 86.9 & 137.0 & 183.1 & 208.4 & 240.2 \\
\hline Taxes on international trade & 34.2 & 34.2 & 47.2 & 44.8 & 8.6 & 17.3 & 27.9 & 37.8 & 40.9 & 44.5 \\
\hline Nontax revenue & 15.7 & 17.8 & 27.2 & 58.5 & 7.4 & 15.0 & 24.1 & 33.5 & 37.6 & 42.2 \\
\hline ID receipts & $\ldots$ & $\ldots$ & 4.0 & 4.0 & 0.8 & 1.5 & $\ldots$ & 1.5 & $\ldots$ & $\ldots$ \\
\hline Grants & 154.8 & 227.9 & 316.8 & 281.5 & 114.6 & 203.9 & 285.0 & 342.2 & 289.4 & 331.8 \\
\hline Budgetary grants & 101.4 & 168.1 & 188.1 & 190.8 & 87.5 & 150.0 & 195.5 & 215.8 & 169.2 & 185.3 \\
\hline Of which: HIPC Initiative assistance & 7.2 & 1.4 & 3.4 & 2.4 & 0.0 & 1.2 & 1.2 & 3.0 & 3.2 & 3.4 \\
\hline Capital grants & 53.4 & 59.8 & 128.7 & 90.7 & 27.1 & 53.9 & 89.5 & 126.4 & 120.2 & 146.5 \\
\hline Project grants & 53.4 & 59.8 & 128.7 & 90.7 & 27.1 & 53.9 & 89.5 & 126.4 & 120.2 & 146.5 \\
\hline Total expenditure and net lending & 414.2 & 521.8 & 711.5 & 738.9 & 207.8 & 416.2 & 612.4 & 814.4 & 879.3 & 963.0 \\
\hline Total expenditure and net lending excluding privatization receipts & 414.2 & 547.5 & 711.5 & 738.9 & 207.8 & 416.2 & 612.4 & 814.4 & 879.3 & 963.0 \\
\hline Current expenditure & 266.5 & 349.0 & 400.7 & 415.2 & 113.3 & 227.4 & 342.5 & 459.3 & 518.9 & 582.3 \\
\hline Of which: priority & 139.7 & 151.7 & 226.5 & 218.0 & 64.6 & 128.7 & 193.9 & 259.9 & $\ldots$ & \\
\hline Wages and salaries & 71.5 & 76.3 & 92.3 & 91.6 & 26.9 & 53.9 & 80.8 & 107.7 & 121.1 & 136.0 \\
\hline Civil & 48.5 & 55.5 & 66.8 & 66.4 & 19.6 & 39.2 & 58.8 & 78.4 & 88.1 & 98.9 \\
\hline Defense & 22.9 & 20.8 & 25.5 & 25.1 & 7.3 & 14.7 & 22.0 & 29.3 & 33.0 & 37.1 \\
\hline Purchases of goods and services & 66.4 & 81.6 & 93.6 & 107.5 & 27.6 & 54.7 & 81.1 & 107.5 & 122.7 & 138.0 \\
\hline Civil & 57.5 & 70.4 & 79.0 & 87.4 & 24.6 & 48.1 & 71.3 & 94.5 & 107.9 & 121.3 \\
\hline Defense & 8.9 & 11.2 & 14.6 & 20.1 & 3.0 & 6.6 & 9.7 & 13.0 & 14.8 & 16.7 \\
\hline Interest payments & 12.8 & 13.6 & 13.7 & 12.4 & 3.2 & 6.9 & 9.7 & 13.4 & 14.0 & 14.1 \\
\hline Domestic debt (due) & 8.7 & 11.1 & 10.4 & 8.4 & 2.3 & 4.4 & 6.5 & 8.6 & 8.7 & 8.8 \\
\hline External debt (due) & 4.1 & 2.4 & 3.3 & 4.0 & 0.9 & 2.5 & 3.2 & 4.8 & 5.3 & 5.3 \\
\hline Transfers & 82.4 & 121.8 & 145.2 & 148.0 & 43.8 & 87.8 & 132.9 & 178.9 & 203.0 & 228.9 \\
\hline Exceptional expenditure & 33.5 & 55.7 & 55.8 & 55.7 & 11.8 & 24.2 & 38.0 & 51.7 & 58.2 & 65.3 \\
\hline Of which: FARG & 10.0 & 14.4 & 13.0 & 12.7 & 4.6 & 9.1 & 14.3 & 20.1 & 20.1 & 20.1 \\
\hline Demobilization & 4.2 & 4.3 & 4.8 & 7.0 & 0.9 & 1.8 & 2.7 & 3.6 & 3.6 & 3.6 \\
\hline African Union peacekeeping & 6.7 & 12.3 & 17.3 & 19.6 & 5.1 & 10.2 & 15.1 & 20.0 & 18.9 & 19.0 \\
\hline Capital expenditure & 131.8 & 188.2 & 298.9 & 307.5 & 92.8 & 185.8 & 265.0 & 348.1 & 353.2 & 373.7 \\
\hline Of which: priority & 28.0 & 38.9 & 108.2 & 123.3 & 42.0 & 85.6 & 110.9 & 137.4 & & \\
\hline Domestic & 42.5 & 87.6 & 130.8 & 140.5 & 46.8 & 95.4 & 123.5 & 153.1 & 171.2 & 192.2 \\
\hline Foreign ${ }^{<}$ & 89.3 & 100.6 & 168.1 & 167.0 & 46.0 & 90.5 & 141.5 & 195.0 & 182.0 & 181.5 \\
\hline Net lending and privatization receipts ${ }^{3}$ & 15.9 & -15.4 & 11.9 & 16.2 & 1.6 & 2.9 & 4.9 & 7.0 & 7.2 & 7.0 \\
\hline Of which: priority & 4.8 & 3.9 & 2.6 & 2.1 & 0.5 & 0.9 & 1.6 & 2.4 & $\ldots$ & $\ldots$ \\
\hline Of which: Rwandatel net receipts & & 25.7 & & & & & & & & \\
\hline \multicolumn{11}{|l|}{ Of which: BK receipts } \\
\hline Primary balance ${ }^{4}$ & -29.5 & -77.1 & -100.0 & -73.2 & -55.0 & -108.7 & -122.3 & -145.8 & -49.6 & -47.3 \\
\hline Domestic fiscal balance $^{5}$ & -87.5 & -128.5 & -178.1 & -153.6 & -70.7 & -140.2 & -171.6 & -213.1 & -236.7 & -255.4 \\
\hline Excluding demobilization and peacekeeping expenditures ${ }^{6}$ & -76.6 & -112.0 & -156.0 & -126.9 & -64.7 & -128.2 & -153.8 & -189.5 & -214.2 & -232.8 \\
\hline Net liquidity impact' & -26.5 & -78.7 & -50.4 & -50.1 & -25.8 & -51.5 & -50.9 & -58.9 & -74.6 & -73.1 \\
\hline Including nonbank domestic debt operations & -17.3 & -96.9 & -47.1 & -67.8 & -28.3 & -56.4 & -56.9 & -66.8 & -74.6 & -73.1 \\
\hline \multicolumn{11}{|l|}{ Overall deficit (payment order) } \\
\hline After grants & -26.1 & -3.6 & -32.7 & -43.0 & -3.0 & -29.2 & -31.3 & -70.7 & -134.6 & -110.3 \\
\hline Before grants & -180.9 & -231.6 & -349.5 & -324.5 & -117.6 & -233.2 & -316.3 & -412.9 & -424.0 & -442.1 \\
\hline Change in arrears ${ }^{8}$ & -7.0 & -8.6 & -5.8 & -7.3 & -2.1 & -5.1 & -6.7 & -8.4 & -7.0 & -7.0 \\
\hline Domestic & -7.0 & -8.6 & -5.8 & -7.3 & -2.1 & -5.1 & -6.7 & -8.4 & -7.0 & -7.0 \\
\hline External & 0.0 & 0.0 & 0.0 & 0.0 & 0.0 & 0.0 & 0.0 & 0.0 & 0.0 & 0.0 \\
\hline Deficit (-) & -33.1 & -12.2 & -38.5 & -50.3 & -5.1 & -34.3 & -38.0 & -79.1 & -141.6 & -117.3 \\
\hline
\end{tabular}


Table 2b. Rwanda: Operations of the Central Government, Fiscal Year Basis ${ }^{1}$, 2006/07-2011/12 (concluded)

\begin{tabular}{|c|c|c|c|c|c|c|c|c|c|c|}
\hline & \multirow[t]{3}{*}{$2006 / 07$} & \multirow[t]{3}{*}{$2007 / 08$} & \multirow{2}{*}{\multicolumn{2}{|c|}{$\begin{array}{r}2008 / 09 \\
\text { Total } \\
\end{array}$}} & \multicolumn{4}{|c|}{$2009 / 10$} & \multirow{3}{*}{$\begin{array}{r}2010 / 11 \\
\text { Proj. }\end{array}$} & \multirow{3}{*}{$\begin{array}{r}2011 / 12 \\
\text { Proj. }\end{array}$} \\
\hline & & & & & \multirow{2}{*}{ Q3 2009} & Q4 2009 & \multirow[t]{2}{*}{ Q1 2010} & \multirow{2}{*}{$\frac{\text { Total }}{\text { Proj. }}$} & & \\
\hline & & & Prog. & Proj. & & Projection & & & & \\
\hline \multicolumn{11}{|c|}{ (Billions of Rwandan francs) } \\
\hline Financing & 40.7 & 11.3 & 38.5 & 50.2 & 5.1 & 34.3 & 38.0 & 79.1 & 141.6 & 117.3 \\
\hline Foreign financing (net) & 38.7 & 42.8 & 33.8 & 72.6 & 18.2 & 34.5 & 49.2 & 63.3 & 141.6 & 117.3 \\
\hline Drawings & 44.2 & 47.9 & 40.2 & 77.0 & 19.0 & 36.6 & 52.0 & 68.6 & 148.9 & 127.6 \\
\hline Budgetary loans & 8.3 & 5.2 & 0.7 & 0.7 & 0.0 & 0.0 & 0.0 & 0.0 & 87.1 & 92.6 \\
\hline Project loans & 36.0 & 42.7 & 39.5 & 76.3 & 19.0 & 36.6 & 52.0 & 68.6 & 61.8 & 35.0 \\
\hline Amortization & -5.6 & -5.1 & -6.3 & -4.4 & -0.8 & -2.1 & -2.8 & -5.3 & -7.3 & -10.3 \\
\hline Exceptional financing & 0.0 & 0.0 & 0.0 & 0.0 & 0.0 & 0.0 & 0.0 & 0.0 & 0.0 & 0.0 \\
\hline Net domestic financing & 2.1 & -31.5 & 4.7 & -22.3 & -13.1 & -0.2 & -11.1 & 15.8 & 0.0 & 0.0 \\
\hline Net credit from banking system & -7.2 & -13.4 & 1.4 & -4.6 & -10.6 & 4.7 & -5.2 & 23.7 & 0.0 & 0.0 \\
\hline Nonbank sector (including CSR repayment) ${ }^{9}$ & 9.3 & -18.1 & 3.3 & -17.7 & -2.5 & -4.9 & -6.0 & -7.9 & 0.0 & 0.0 \\
\hline Errors and omissions / Financing gap ${ }^{10}$ & 7.6 & -0.9 & 0.1 & -0.1 & 0.0 & 0.0 & 0.0 & 0.0 & 0.0 & 0.0 \\
\hline \multicolumn{11}{|c|}{ (Percent of GDP, unless otherwise indicated) } \\
\hline Memorandum items: & & & & & & & & & & \\
\hline Revenue and grants & 22.6 & 24.1 & 26.8 & 26.2 & 6.7 & 12.7 & 19.1 & 24.4 & 21.9 & 22.5 \\
\hline Grants & 9.0 & 10.6 & 12.5 & 10.6 & 3.8 & 6.7 & 9.4 & 11.2 & 8.5 & 8.7 \\
\hline Revenue, excluding grants & 13.6 & 13.5 & 14.3 & 15.6 & 3.0 & 6.0 & 9.7 & 13.2 & 13.4 & 13.7 \\
\hline Revenue, excluding grants (percent of monetized sector GDP) & 21.2 & 21.0 & 22.8 & 24.8 & 4.8 & 9.7 & 15.6 & 21.3 & 21.7 & 22.1 \\
\hline Total expenditure and net lending & 24.1 & 24.3 & 28.1 & 27.8 & 6.8 & 13.7 & 20.1 & 26.7 & 25.8 & 25.4 \\
\hline Total expenditure and net lending excluding privatization receipts & 24.1 & 25.4 & 28.1 & 27.8 & 6.8 & 13.7 & 20.1 & 26.7 & 25.8 & 25.4 \\
\hline Of which: total priority expenditures & 10.1 & 9.0 & 13.3 & 12.9 & 3.5 & 7.1 & 10.1 & 13.1 & $\ldots$ & $\ldots$ \\
\hline Total expenditure & 23.2 & 25.0 & 27.6 & 27.2 & 6.8 & 13.6 & 19.9 & 26.5 & 25.6 & 25.2 \\
\hline Current expenditure & 15.5 & 16.2 & 15.8 & 15.6 & 3.7 & 7.5 & 11.2 & 15.1 & 15.2 & 15.3 \\
\hline Of which: wage bill & 4.2 & 3.5 & 3.6 & 3.4 & 0.9 & 1.8 & 2.7 & 3.5 & 3.6 & 3.6 \\
\hline goods and services & 3.9 & 3.8 & 3.7 & 4.0 & 0.9 & 1.8 & 2.7 & 3.5 & 3.6 & 3.6 \\
\hline Of which: defense/security & 1.9 & 1.5 & 1.6 & 1.7 & 0.3 & 0.7 & 1.0 & 1.4 & 1.4 & 1.4 \\
\hline exceptional expenditure & 2.0 & 2.6 & 2.2 & 2.1 & 0.4 & 0.8 & 1.2 & 1.7 & 1.7 & 1.7 \\
\hline Capital expenditure & 7.7 & 8.7 & 11.8 & 11.6 & 3.0 & 6.1 & 8.7 & 11.4 & 10.4 & 9.8 \\
\hline Domestic fiscal balance & -5.1 & -6.0 & -7.0 & -5.8 & -2.3 & -4.6 & -5.6 & -7.0 & -7.0 & -6.7 \\
\hline Excluding demobilization and peacekeeping expenditures ${ }^{5}$ & -4.5 & -5.2 & -6.2 & -4.8 & -2.1 & -4.2 & -5.0 & -6.2 & -6.3 & -6.1 \\
\hline Of which: domestic expenditure & 18.1 & 18.7 & 20.4 & 20.4 & 5.1 & 10.2 & 14.8 & 19.4 & 19.7 & 19.9 \\
\hline Net liquidity impact ${ }^{7}$ & -1.5 & -3.7 & -2.0 & -1.9 & -0.8 & -1.7 & -1.7 & -1.9 & -2.2 & -1.9 \\
\hline Including nonbank domestic debt operations & -1.0 & -4.5 & -1.9 & -2.6 & -0.9 & -1.9 & -1.9 & -2.2 & -2.2 & -1.9 \\
\hline Primary balance ${ }^{4}$ & -1.7 & -3.6 & -3.9 & -2.8 & -1.8 & -3.6 & -4.0 & -4.8 & -4.8 & -4.6 \\
\hline \multicolumn{11}{|l|}{ Overall deficit (payment order) } \\
\hline After grants & -1.5 & -0.2 & -1.3 & -1.6 & -0.1 & -1.0 & -1.0 & -2.3 & -4.0 & -2.9 \\
\hline Before grants & -10.5 & -10.8 & -13.8 & -12.2 & -3.9 & -7.7 & -10.4 & -13.6 & -12.5 & -11.6 \\
\hline Net domestic financing & 0.1 & -1.5 & 0.2 & -0.8 & -0.4 & 0.0 & -0.4 & 0.5 & 0.0 & 0.0 \\
\hline Net credit from banking system & -0.4 & -0.6 & 0.1 & -0.2 & -0.3 & 0.2 & -0.2 & 0.8 & 0.0 & 0.0 \\
\hline Nominal GDP (RF billions) & $1,715.0$ & $2,151.7$ & $2,535.4$ & $2,657.4$ & $3,046.4$ & $3,046.4$ & $3,046.4$ & $3,046.4$ & $3,403.2$ & $3,796.4$ \\
\hline Of which: monetized sectors & 908.1 & $1,380.9$ & $1,591.3$ & $1,667.9$ & $1,895.4$ & $1,895.4$ & $1,895.4$ & $1,888.1$ & $2,102.4$ & $2,352.1$ \\
\hline
\end{tabular}

Sources: Rwandan authorities, and IMF staff estimates and projections.

${ }^{1}$ Fiscal year runs from July to June.

${ }^{2}$ For 2008/09, the increase partially reflects improvements in recording funds for projects.

${ }^{3}$ Net lending in 2007/08 incorporates RF35.7 billion in receipts from the privatization of Rwandatel.

${ }^{4}$ Definition excludes exceptional expenditures, defined as total revenue (excluding privatization proceeds) minus noninterest current

expenditure (excluding exceptional expenditure) minus domestically financed capital expenditure.

${ }^{5}$ Revenue excluding grants minus current expenditure,

${ }^{6}$ For $2006 / 07$, peacekeeping activities were not fully covered by grants.

7 Total expenditure and net lending (excluding privatization receipts) less revenues, foreign financed project spending,

and current spending on imports.

${ }^{8}$ A negative sign indicates a reduction.

${ }^{9} \mathrm{CSR}=$ Caisse Sociale du Rwanda.

${ }^{10}$ A negative number implies an underestimate of financing. 
Table 3. Rwanda: Monetary Survey, 2006-10

\begin{tabular}{|c|c|c|c|c|c|c|c|c|c|c|c|c|c|c|c|}
\hline & \multirow{3}{*}{2006} & \multirow{3}{*}{2007} & \multicolumn{5}{|c|}{2008} & \multirow{3}{*}{$\begin{array}{r}\frac{2008^{1}}{2 \text { December }} \\
\text { Act. }\end{array}$} & \multicolumn{5}{|c|}{2009} & \multicolumn{2}{|c|}{2010} \\
\hline & & & \multirow[t]{2}{*}{ March } & \multirow[t]{2}{*}{ June } & \multirow[t]{2}{*}{ Sept. } & \multicolumn{2}{|c|}{ December } & & \multicolumn{2}{|c|}{ March } & \multirow{2}{*}{$\frac{\text { June }}{\text { Proj. }}$} & \multirow{2}{*}{$\frac{\text { Sept. }}{\text { Proj. }}$} & \multirow{2}{*}{$\begin{array}{l}\text { Dec. } \\
\text { Proj. }\end{array}$} & \multirow{2}{*}{$\begin{array}{r}\text { March } \\
\text { Proj. }\end{array}$} & \multirow{2}{*}{$\begin{array}{r}\text { June } \\
\text { Proj. }\end{array}$} \\
\hline & & & & & & Prog. & Act. & & Proj. & Act. & & & & & \\
\hline \multicolumn{16}{|c|}{ (Billions of Rwanda francs) } \\
\hline \multicolumn{16}{|l|}{ Monetary authorities } \\
\hline Net foreign assets & 225.0 & 281.8 & 296.5 & 331.0 & 319.2 & 275.7 & 325.6 & 325.6 & 311.6 & 272.2 & 312.6 & 341.6 & 326.1 & 325.0 & 301.8 \\
\hline Foreign assets & 241.2 & 300.6 & 318.6 & 358.7 & 343.3 & 292.0 & 333.4 & 333.4 & 335.7 & 280.7 & 323.9 & 350.3 & 334.8 & 333.6 & 310.5 \\
\hline Foreign liabilities ${ }^{2}$ & 16.3 & 18.8 & 22.1 & 27.7 & 24.1 & 16.4 & 7.8 & 7.8 & 24.1 & 8.5 & 11.3 & 8.7 & 8.7 & 8.7 & 8.7 \\
\hline Net domestic assets & -153.1 & -187.9 & -191.5 & -220.9 & -206.8 & -167.4 & -209.6 & -209.6 & -184.9 & -160.1 & -202.9 & -227.8 & -209.3 & -205.6 & -176.3 \\
\hline Domestic credit & -119.4 & -152.6 & -154.2 & -189.8 & -165.4 & -131.4 & -168.7 & -168.7 & -154.6 & -116.3 & -154.8 & -179.7 & -159.8 & -156.1 & -127.3 \\
\hline Government (net) & -71.6 & -98.8 & -120.7 & -159.8 & -135.6 & -73.6 & -158.5 & -158.5 & -132.7 & -122.4 & -130.9 & -177.2 & -149.9 & -159.9 & -131.0 \\
\hline Claims & 41.8 & 41.2 & 41.2 & 39.7 & 39.7 & 41.0 & 39.7 & 39.7 & 38.9 & 45.2 & 39.4 & 39.4 & 38.9 & 38.9 & 38.9 \\
\hline Deposits (excluding autonomous bodies) ${ }^{2}$ & 113.4 & 140.0 & 161.9 & 199.5 & 175.4 & 114.6 & 198.3 & 198.3 & 171.6 & 167.6 & 170.3 & 216.6 & 188.8 & 198.8 & 169.9 \\
\hline Public nongovernment deposits (-) & -2.0 & -1.3 & -0.2 & -0.5 & -0.5 & -0.9 & -0.5 & -0.5 & -0.3 & -0.5 & -0.6 & -0.9 & -1.0 & -0.9 & -1.0 \\
\hline Nongovernment credit & -45.8 & -52.5 & -33.3 & -29.5 & -29.3 & -56.9 & -9.7 & -9.7 & -21.6 & 6.6 & -23.3 & -1.7 & -8.8 & 4.8 & 4.7 \\
\hline Private sector & 3.5 & 4.8 & 4.7 & 5.1 & 5.4 & 4.0 & 5.4 & 5.4 & 5.8 & 5.7 & 7.1 & 5.7 & 5.9 & 5.7 & 5.9 \\
\hline Public enterprises & -- & -- & -- & -- & -- & -- & -- & -- & -- & -- & -- & -- & -- & -- & -- \\
\hline Commercial banks & -49.3 & -57.3 & -38.0 & -34.7 & -34.7 & -60.9 & -15.1 & -15.1 & -27.4 & 0.9 & -30.4 & -7.4 & -14.8 & -1.0 & -1.2 \\
\hline Discount window & 1.2 & 1.3 & 1.3 & 1.5 & 1.3 & 1.3 & 1.5 & 1.5 & 1.3 & 0.9 & 1.3 & 1.3 & 0.9 & 0.9 & 0.9 \\
\hline Money market (- = absorption) & -50.5 & -58.6 & -39.2 & -36.2 & -36.0 & -62.2 & -16.6 & -16.6 & -28.7 & -- & -31.7 & -8.7 & -15.7 & -1.9 & -2.1 \\
\hline Other items (net; asset +) & -33.8 & -35.4 & -37.3 & -31.1 & -41.4 & -36.0 & -41.0 & -41.0 & -30.3 & -43.8 & -48.1 & -48.1 & -49.5 & -49.5 & -49.0 \\
\hline Reserve money ${ }^{3,4}$ & 71.8 & 93.9 & 104.9 & 110.1 & 112.4 & 108.3 & 115.9 & 115.9 & 126.7 & 112.1 & 109.7 & 113.8 & 116.9 & 119.4 & 125.6 \\
\hline Currency in circulation & 54.6 & 67.3 & 64.4 & 74.7 & 77.0 & 72.0 & 82.9 & 80.9 & 78.2 & 66.3 & 72.2 & 70.4 & 72.5 & 78.9 & 78.9 \\
\hline Commercial bank reserves & 15.1 & 24.0 & 34.2 & 32.5 & 26.2 & 34.0 & 23.3 & 32.7 & 46.4 & 43.5 & 33.8 & 39.7 & 40.6 & 36.8 & 42.9 \\
\hline Nonbank deposits & 2.1 & 2.6 & 6.4 & 2.9 & 9.1 & 2.3 & 9.8 & 2.3 & 2.1 & 2.3 & 3.7 & 3.7 & 3.7 & 3.7 & 3.7 \\
\hline Of which: autonomous public agencies & 0.4 & 0.1 & -- & -- & -- & -- & -- & -- & -- & -- & -- & -- & -- & -- & -- \\
\hline Commercial banks & & & & & & & & & & & & & & & \\
\hline Net foreign assets & 60.1 & 69.6 & 70.7 & 73.9 & 74.4 & 72.2 & 77.7 & 78.2 & 92.1 & 76.5 & 73.8 & 74.5 & 77.3 & 72.3 & 77.3 \\
\hline Foreign assets & 70.9 & 86.8 & 86.7 & 91.8 & 92.6 & 89.6 & 96.3 & 96.8 & 110.6 & 97.2 & 96.8 & 96.8 & 100.3 & 98.3 & 100.3 \\
\hline Foreign liabilities & 10.8 & 17.2 & 16.0 & 17.9 & 18.2 & 17.4 & 18.6 & 18.6 & 18.5 & 20.7 & 23.0 & 23.0 & 23.0 & 26.0 & 23.0 \\
\hline Reserves & 15.1 & 24.0 & 34.2 & 32.5 & 26.2 & 34.0 & 23.3 & 32.7 & 46.4 & 43.5 & 33.8 & 39.7 & 40.6 & 36.8 & 42.9 \\
\hline NBR deposits & 11.3 & 18.6 & 26.0 & 24.9 & 17.4 & 31.0 & 17.6 & 25.1 & 37.9 & 33.0 & 24.3 & 27.1 & 28.1 & 24.3 & 29.9 \\
\hline Required reserves & 18.8 & 24.4 & 26.3 & 27.1 & 28.1 & 30.4 & 27.4 & 34.2 & 38.3 & 20.3 & 20.7 & 22.0 & 22.0 & 23.3 & 23.5 \\
\hline Excess reserves & -7.5 & -5.8 & -0.3 & -2.2 & -10.7 & 0.6 & -9.8 & -9.1 & -0.4 & 12.7 & 3.7 & 5.0 & 6.2 & 0.9 & 6.5 \\
\hline Cash in vault & 3.8 & 5.4 & 8.1 & 7.6 & 8.9 & 3.0 & 5.6 & 7.6 & 8.5 & 10.5 & 9.5 & 12.6 & 12.5 & 12.5 & 13.0 \\
\hline Net credit from NBR (rediscount; liability -) & 49.3 & 57.3 & 38.0 & 34.7 & 34.7 & 60.9 & 15.1 & 15.1 & 27.4 & -0.9 & 30.4 & 7.4 & 14.8 & 1.0 & 1.2 \\
\hline Domestic credit & 158.7 & 218.5 & 220.1 & 225.3 & 241.2 & 243.4 & 258.4 & 352.9 & 360.7 & 333.4 & 324.7 & 346.2 & 345.9 & 377.7 & 387.1 \\
\hline Government (net) & -2.3 & 25.4 & 19.7 & 13.0 & 8.7 & 0.8 & 15.7 & 16.2 & 11.3 & -2.0 & -4.6 & -6.0 & -12.0 & -12.0 & -12.0 \\
\hline Credit & 25.6 & 40.6 & 37.4 & 30.3 & 24.9 & 33.4 & 27.7 & 29.3 & 27.2 & 23.7 & 16.7 & 21.7 & 21.7 & 21.7 & 21.7 \\
\hline Deposits & 27.9 & 15.2 & 17.6 & 17.2 & 16.2 & 32.6 & 12.0 & 13.1 & 15.9 & 25.7 & 21.3 & 27.7 & 33.7 & 33.7 & 33.7 \\
\hline Public enterprises & 2.4 & 1.7 & 1.4 & 1.4 & 1.5 & 1.7 & 1.6 & 1.6 & 1.5 & 2.6 & 1.4 & 1.4 & 1.4 & 1.4 & 1.4 \\
\hline Private sector & 158.7 & 191.3 & 199.0 & 210.9 & 231.0 & 240.9 & 241.0 & 335.0 & 347.9 & 332.9 & 327.9 & 350.7 & 356.5 & 388.3 & 397.7 \\
\hline Other items (net; asset + ) & -53.1 & -64.1 & -70.1 & -72.7 & -63.5 & -62.5 & -71.4 & -96.0 & -63.8 & -94.9 & -99.0 & -81.0 & -99.0 & -81.0 & -99.0 \\
\hline Deposits & 230.2 & 305.3 & 292.8 & 293.7 & 312.9 & 348.0 & 303.1 & 382.9 & 462.8 & 358.4 & 363.7 & 386.7 & 379.6 & 406.8 & 409.4 \\
\hline Private & 185.8 & 246.9 & 242.8 & 248.6 & 255.1 & 289.6 & 248.1 & 326.0 & 397.3 & 306.7 & 310.0 & 332.0 & 325.7 & 349.4 & 350.8 \\
\hline Public (nongovernment) & 44.4 & 58.4 & 50.1 & 45.2 & 57.8 & 58.4 & 55.0 & 56.9 & 65.5 & 51.7 & 53.7 & 54.7 & 53.9 & 57.4 & 58.6 \\
\hline
\end{tabular}


Table 3. Rwanda: Monetary Survey, 2006-10 (concluded)

\begin{tabular}{|c|c|c|c|c|c|c|c|c|c|c|c|c|c|c|c|}
\hline & \multirow[t]{3}{*}{$2006^{1}$} & \multirow[t]{3}{*}{2007} & \multicolumn{5}{|c|}{2008} & \multirow{3}{*}{ 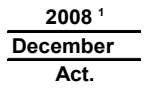 } & \multicolumn{5}{|c|}{2009} & \multicolumn{2}{|c|}{2010} \\
\hline & & & \multirow{2}{*}{$\begin{array}{l}\text { March } \\
\text { Act. }\end{array}$} & \multirow{2}{*}{$\begin{array}{l}\text { June } \\
\text { Act. }\end{array}$} & \multirow{2}{*}{$\begin{array}{l}\text { Sept. } \\
\text { Act. }\end{array}$} & \multicolumn{2}{|c|}{ December } & & \multicolumn{2}{|c|}{ March } & \multirow{2}{*}{$\begin{array}{c}\text { June } \\
\text { Proj. }\end{array}$} & \multirow{2}{*}{$\begin{array}{c}\text { Sept. } \\
\text { Proj. }\end{array}$} & \multirow{2}{*}{$\begin{array}{l}\text { Dec. } \\
\text { Proj. }\end{array}$} & \multirow{2}{*}{$\begin{array}{r}\text { March } \\
\text { Proj. }\end{array}$} & \multirow{2}{*}{$\begin{array}{c}\text { June } \\
\text { Proj }\end{array}$} \\
\hline & & & & & & Prog. & Act. & & Proj. & Act. & & & & & \\
\hline \multicolumn{16}{|c|}{ (Billions of Rwanda francs) } \\
\hline Net foreign assets & 285.1 & 351.5 & 367.1 & 404.9 & 393.6 & 347.9 & 403.3 & 403.8 & 403.8 & 348.7 & 386.4 & 416.1 & 403.4 & 397.3 & 379.1 \\
\hline Net domestic assets & 1.9 & 23.8 & -3.5 & -33.6 & 5.5 & 74.4 & -7.5 & 62.3 & 139.3 & 77.6 & 53.2 & 44.7 & 52.4 & 92.1 & 113.0 \\
\hline Domestic credit & 88.7 & 123.2 & 103.8 & 70.2 & 110.4 & 172.9 & 104.8 & 199.3 & 233.4 & 216.2 & 200.3 & 173.8 & 200.9 & 222.6 & 261.0 \\
\hline Government (net) & -73.9 & -73.4 & -101.0 & -146.7 & -127.0 & -72.8 & -142.8 & -142.3 & -121.4 & -124.4 & -135.5 & -183.2 & -161.9 & -171.9 & -143.0 \\
\hline Public nongovernment deposits (-) & -2.0 & -1.3 & -0.2 & -0.5 & -0.5 & -0.9 & -0.5 & -0.5 & -0.3 & -0.5 & -0.6 & -0.9 & -1.0 & -0.9 & -1.0 \\
\hline Public enterprises & 2.4 & 1.8 & 1.4 & 1.4 & 1.5 & 1.7 & 1.6 & 1.6 & 1.5 & 2.6 & 1.4 & 1.4 & 1.4 & 1.4 & 1.4 \\
\hline Private sector & 162.2 & 196.2 & 203.6 & 216.0 & 236.3 & 244.9 & 246.5 & 340.5 & 353.7 & 338.6 & 335.0 & 356.5 & 362.5 & 394.0 & 403.6 \\
\hline Other items (net; asset +) & -86.8 & -99.4 & -107.4 & -103.8 & -104.9 & -98.5 & -112.3 & -137.0 & -94.1 & -138.7 & -147.1 & -129.1 & -148.5 & -130.5 & -148.0 \\
\hline Broad money $y^{5,6}$ & 287.0 & 375.2 & 363.6 & 371.3 & 399.1 & 422.3 & 395.8 & 466.1 & 543.1 & 427.0 & 439.6 & 460.8 & 455.9 & 489.4 & 492.1 \\
\hline Currency in circulation & 54.6 & 67.3 & 64.4 & 74.7 & 77.0 & 72.0 & 82.9 & 80.9 & 78.2 & 66.3 & 72.2 & 70.4 & 72.5 & 78.9 & 78.9 \\
\hline Deposits & 232.3 & 307.9 & 299.2 & 296.6 & 322.0 & 350.3 & 312.9 & 385.2 & 464.9 & 360.7 & 367.4 & 390.4 & 383.3 & 410.5 & 413.1 \\
\hline Of which: foreign currency deposits & 52.5 & 62.7 & 64.6 & 67.4 & 70.3 & 72.2 & 74.7 & 74.7 & 91.9 & 71.6 & 86.0 & 74.5 & 86.0 & 85.6 & 85.4 \\
\hline \multicolumn{16}{|c|}{ (Annual changes in percent of beginning-of-period broad money) } \\
\hline Net foreign assets & 28.7 & 23.1 & 38.3 & 18.7 & 22.4 & -1.9 & 13.8 & 13.9 & 8.2 & -5.1 & -7.3 & 4.9 & 1.9 & 9.7 & -5.1 \\
\hline Net domestic assets & 2.6 & 7.6 & -7.0 & 2.5 & -2.0 & 14.3 & -8.3 & 10.3 & 40.7 & 22.3 & 25.2 & 10.4 & -3.9 & 4.5 & 16.2 \\
\hline Domestic credit & 8.2 & 12.0 & -5.4 & 1.5 & -2.9 & 15.2 & -4.9 & 20.5 & 35.5 & 30.9 & 34.9 & 15.9 & 0.4 & 0.1 & 13.7 \\
\hline Government (net) & -5.6 & 0.2 & -16.6 & -9.9 & -15.7 & 2.1 & -18.5 & -18.1 & -5.6 & -6.4 & 3.0 & -14.1 & -4.2 & -12.3 & -1.7 \\
\hline Economy & 13.8 & 11.9 & 11.2 & 11.5 & 12.8 & 13.1 & 13.6 & 38.6 & 41.1 & 37.4 & 31.9 & 29.9 & 4.6 & 12.4 & 15.4 \\
\hline Other items (net; asset +) & -5.6 & -4.4 & -1.6 & 1.0 & 0.9 & -0.9 & -3.4 & -10.2 & 5.1 & -8.6 & -9.7 & -5.5 & -4.2 & 4.4 & 2.5 \\
\hline Broad money & 31.3 & 30.8 & 31.2 & 21.2 & 20.4 & 12.3 & 5.5 & 24.2 & 48.9 & 17.4 & 17.9 & 15.3 & -2.4 & 14.2 & 11.1 \\
\hline \multicolumn{16}{|c|}{ (Annual percent changes) } \\
\hline Net foreign assets & 28.2 & 23.3 & 40.6 & 16.5 & 23.3 & -2.0 & 14.7 & 14.9 & 8.0 & -5.0 & -6.5 & 1.0 & 2.3 & 11.7 & -5.6 \\
\hline Net domestic assets & -150.0 & $1,163.8$ & -122.0 & -18.6 & -54.2 & 258.4 & -131.6 & 162.1 & $-4,061.5$ & $-2,305.9$ & -253.5 & 360.6 & -25.5 & 2.8 & 112.3 \\
\hline Domestic credit & 25.2 & 38.9 & -12.6 & 7.2 & -8.0 & 49.4 & -14.9 & 62.8 & 124.9 & 108.2 & 185.2 & 57.4 & 0.8 & 0.2 & 30.3 \\
\hline Government (net) & 20.0 & -0.7 & 84.1 & 26.2 & 69.5 & -10.0 & 94.5 & 91.7 & 20.2 & 23.2 & -7.7 & 44.3 & 13.8 & 43.9 & 5.6 \\
\hline Economy & 22.8 & 20.9 & 17.9 & 19.3 & 21.8 & 25.0 & 25.9 & 73.7 & 73.2 & 66.3 & 54.8 & 50.4 & 6.2 & 15.5 & 20.3 \\
\hline Other items (net; asset + ) & 16.3 & 14.5 & 4.4 & -2.8 & -2.9 & 3.7 & 13.0 & 38.9 & -16.6 & 29.1 & 32.4 & 8.2 & 15.2 & -8.9 & -6.9 \\
\hline \multicolumn{16}{|c|}{ (In percent, unless otherwise indicated) } \\
\hline Memorandum items: & & & & & & & & & & & & & & & \\
\hline Currency/broad money ratio & 19.0 & 17.9 & 17.7 & 20.1 & 19.3 & 17.0 & 20.9 & 17.4 & 14.4 & 15.5 & 16.4 & 16.1 & 15.9 & 16.1 & 16.0 \\
\hline Reserve money annual growth & 11.9 & 30.7 & 31.7 & 23.0 & 24.6 & 15.4 & 23.5 & 23.5 & 20.7 & 6.8 & -0.4 & 6.5 & 0.8 & 6.5 & 14.5 \\
\hline Reserves/deposits & 31.2 & 30.8 & 35.8 & 37.5 & 35.9 & 31.1 & 38.3 & 30.3 & 27.4 & 31.3 & 30.2 & 29.4 & 30.8 & 29.4 & 30.7 \\
\hline Money multiplier & 4.0 & 4.0 & 3.5 & 3.4 & 3.6 & 3.9 & 3.4 & 4.0 & 4.3 & 3.8 & 4.0 & 4.1 & 3.9 & 4.1 & 3.9 \\
\hline Velocity of broad money (end of period) & 5.4 & 5.0 & 6.7 & 6.6 & 6.1 & 4.9 & 6.2 & 5.2 & 4.6 & 6.7 & 6.5 & 6.5 & 6.3 & 6.2 & 6.2 \\
\hline Net open position of the NBR (RF billion) & 219.1 & 275.6 & 289.7 & 324.0 & 310.7 & 269.9 & 317.9 & 317.9 & 304.3 & 267.6 & 307.4 & 319.8 & 321.0 & 319.8 & 296.7 \\
\hline Net open position of commercial banks (RF billion) & 13.5 & 13.2 & 12.9 & 13.5 & 12.6 & 2.0 & 10.7 & 11.2 & 0.2 & 9.4 & -10.5 & 2.2 & 0.0 & 2.2 & 0.0 \\
\hline Extended broad money (RF billion) ${ }^{7}$ & 333.8 & 438.2 & 431.2 & 444.1 & 477.1 & 491.1 & 473.7 & 473.7 & $\ldots$ & $\ldots$ & $\ldots$ & $\ldots$ & & $\ldots$ & \\
\hline Nominal GDP (RF billion) & $1,563.8$ & $1,866.1$ & $\ldots$ & & & $2,081.1$ & $2,437.2$ & $2,437.2$ & $\ldots$ & & $\ldots$ & & $2,877.5$ & $\ldots$ & $3,591.1$ \\
\hline
\end{tabular}

Source: National Bank of Rwanda (NBR); and IMF staff estimates and projections.

1 Reflects the operations of the Union Banques Populaires du Rwanda (UBPR)—a large microfinance network— which was transformed into a commercial bank in Febuary 2008.

${ }^{2}$ The IMF's MDRI reduced foreign liabilities at the NBR by RF 42.4 million with a counter entry in government deposits (in January 2006).

${ }^{3}$ The definition of reserve money as performance criterion or structural benchmark differs from the definition in the monetary program in that it excludes the deposits of a defunct savings bank, import

deposits, and dormant accounts. It includes old notes demonetized at the end of 2004 but in circulation until the end of 2005.

The reserve money target for 2007 is derived by applying the programmed growth rate of 13 percent to the rebased 2006 outcome. The rebased 2006 outcome was calculated by allowing banks to meet the legal reserve requirement on deposits (banks' reserves at the end of December 2006 fell short of the requirement by RF 7.5 billion, they met the requirement because it is based on an average over two weeks;

plus (2) a liquidhy resenve

${ }^{5}$ From December 2005 on, includes Caisse Hypothecaire du Rwanda (BHR) deposits (RF 1 billion at end-2005)

${ }^{6}$ End-2006 broad money includes RF5 billion temporary build up of local government deposits, which were unwound by February 2007.

${ }^{7}$ Broad money plus deposits in the Union de Banques Populaires de Rwanda (UBPR) through December 2008 and Rwanda Development Bank (BRD). 
Table 4. Rwanda: Balance of Payments, 2006-14

(Millions of U.S. dollars, unless otherwise indicated)

\begin{tabular}{|c|c|c|c|c|c|c|c|c|c|}
\hline & \multirow[t]{2}{*}{2006} & \multirow{2}{*}{$\begin{array}{r}2007 \\
\text { Est. }\end{array}$} & \multirow{2}{*}{$\begin{array}{r}2008 \\
\text { Est. }\end{array}$} & \multirow{2}{*}{$\frac{2009}{\text { Proj. }}$} & 2010 & 2011 & 2012 & 2013 & 2014 \\
\hline & & & & & \\
\hline Exports, f.o.b. & 147.3 & 176.8 & 256.5 & 198.5 & 244.0 & 280.6 & 318.3 & 351.0 & 387.3 \\
\hline Of which: coffee & 54.0 & 35.7 & 46.9 & 50.6 & 65.1 & 62.5 & 67.5 & 71.1 & 73.3 \\
\hline tea & 31.9 & 31.5 & 40.1 & 47.9 & 55.2 & 66.5 & 73.5 & 81.3 & 89.8 \\
\hline Imports, f.o.b. & 446.4 & 581.2 & 880.7 & 831.0 & 894.4 & 947.8 & 1016.0 & 1106.7 & 1220.3 \\
\hline Trade balance & -299.1 & -404.4 & -624.2 & -632.5 & -650.4 & -667.2 & -697.7 & -755.6 & -833.0 \\
\hline Services (net)' & -132.3 & -123.2 & -102.8 & -269.3 & -252.7 & -214.2 & -191.0 & -158.0 & -93.1 \\
\hline $\begin{array}{l}\text { Income } \\
\text { Of which: interest on public debt }{ }^{2}\end{array}$ & $\begin{array}{l}-28.7 \\
-10.2\end{array}$ & $\begin{array}{r}-17.2 \\
-6.3\end{array}$ & $\begin{array}{r}-35.1 \\
-6.7\end{array}$ & $\begin{array}{r}-61.7 \\
-7.2\end{array}$ & $\begin{array}{r}-66.0 \\
-7.5\end{array}$ & $\begin{array}{r}-70.7 \\
-9.5\end{array}$ & $\begin{array}{l}-68.4 \\
-10.8\end{array}$ & $\begin{array}{l}-73.5 \\
-12.2\end{array}$ & $\begin{array}{l}-77.8 \\
-13.7\end{array}$ \\
\hline $\begin{array}{l}\text { Current transfers (net) })^{3} \\
\quad \text { Private }\end{array}$ & $\begin{array}{r}325.8 \\
77.2\end{array}$ & $\begin{array}{r}461.3 \\
98.8\end{array}$ & $\begin{array}{r}518.5 \\
72.6\end{array}$ & $\begin{array}{r}634.2 \\
73.8\end{array}$ & $\begin{array}{r}466.4 \\
77.3\end{array}$ & $\begin{array}{r}438.0 \\
83.9\end{array}$ & $\begin{array}{r}431.4 \\
91.3\end{array}$ & $\begin{array}{r}452.5 \\
99.3\end{array}$ & $\begin{array}{l}475.8 \\
108.1\end{array}$ \\
\hline $\begin{array}{l}\text { Public } \\
\text { Of which: HIPC grants }\end{array}$ & $\begin{array}{r}248.7 \\
22.2\end{array}$ & $\begin{array}{r}362.5 \\
4.5\end{array}$ & $\begin{array}{r}445.9 \\
6.6\end{array}$ & $\begin{array}{r}560.4 \\
5.2\end{array}$ & $\begin{array}{r}389.2 \\
4.9\end{array}$ & $\begin{array}{r}354.1 \\
4.5\end{array}$ & $\begin{array}{r}340.1 \\
5.0\end{array}$ & $\begin{array}{r}353.2 \\
5.5\end{array}$ & $\begin{array}{r}367.7 \\
5.6\end{array}$ \\
\hline $\begin{array}{l}\text { Current account balance (including official transfers) } \\
\text { Current account balance (excluding official transfers) }\end{array}$ & $\begin{array}{l}-134.3 \\
-383.0\end{array}$ & $\begin{array}{r}-83.5 \\
-446.0\end{array}$ & $\begin{array}{l}-243.5 \\
-689.4\end{array}$ & $\begin{array}{l}-329.2 \\
-889.6\end{array}$ & $\begin{array}{l}-502.6 \\
-891.8\end{array}$ & $\begin{array}{l}-514.1 \\
-868.2\end{array}$ & $\begin{array}{l}-525.8 \\
-865.8\end{array}$ & $\begin{array}{l}-534.6 \\
-887.8\end{array}$ & $\begin{array}{l}-528.1 \\
-895.8\end{array}$ \\
\hline $\begin{array}{l}\text { Capital account } \\
\text { Capital transfers }\end{array}$ & $\begin{array}{r}1323.7 \\
173.7\end{array}$ & $\begin{array}{l}92.3 \\
92.3\end{array}$ & $\begin{array}{l}172.1 \\
172.1\end{array}$ & $\begin{array}{l}148.3 \\
148.3\end{array}$ & $\begin{array}{l}211.8 \\
211.8\end{array}$ & $\begin{array}{l}209.8 \\
209.8\end{array}$ & $\begin{array}{l}190.9 \\
190.9\end{array}$ & $\begin{array}{l}194.6 \\
194.6\end{array}$ & $\begin{array}{l}198.4 \\
198.4\end{array}$ \\
\hline IMF-MDRI relief & 76.6 & & & & & & & & \\
\hline Debt forgiveness (IDA and AfDF-MDRI Relief) & 1150.0 & & & & & & & & \\
\hline Financial account & -1109.9 & 180.5 & 182.1 & 162.0 & 261.8 & 300.0 & 344.7 & 381.4 & 389.9 \\
\hline Direct investment & 30.6 & 82.3 & 103.4 & 65.0 & 91.0 & 104.7 & 120.3 & 132.4 & 139.0 \\
\hline Public sector capital & 46.1 & 79.9 & 103.2 & 117.3 & 142.7 & 193.3 & 206.2 & 214.1 & 224.5 \\
\hline Long-term borrowing ${ }^{4}$ & 68.4 & 88.2 & 110.5 & 128.5 & 154.6 & 209.7 & 226.1 & 236.2 & 247.3 \\
\hline Scheduled amortization $^{5}$ & -22.3 & -8.3 & -7.3 & -11.2 & -11.9 & -16.4 & -19.9 & -22.1 & -22.9 \\
\hline Other capital $^{6}$ & -1186.5 & 18.3 & -24.4 & -20.3 & 28.1 & 2.1 & 18.1 & 34.9 & 26.5 \\
\hline Capital and financial account balance & 213.8 & 272.9 & 354.2 & 310.4 & 473.6 & 509.8 & 535.6 & 576.0 & 588.3 \\
\hline Errors and omissions/Financing gap & 3.0 & -81.5 & -46.0 & 0.0 & 0.0 & 0.0 & 0.0 & 0.0 & 0.0 \\
\hline Overall balance & 82.5 & 107.9 & 64.7 & -18.8 & -29.0 & -4.3 & 9.8 & 41.4 & 60.2 \\
\hline Financing & -82.5 & -107.9 & -64.7 & 17.1 & 29.0 & 4.3 & -9.8 & -41.4 & -60.2 \\
\hline Change in net foreign assets of NBR (increase -) & -82.5 & -107.9 & -64.7 & 17.1 & 29.0 & 4.3 & -9.8 & -41.4 & -60.2 \\
\hline Net credit from the Fund & -72.5 & 3.4 & 3.7 & 1.7 & -0.1 & -0.6 & -1.0 & -1.7 & -2.5 \\
\hline Disbursements/purchases & 4.1 & 3.4 & 3.7 & 1.7 & 0.0 & 0.0 & 0.0 & 0.0 & 0.0 \\
\hline Repayments/repurchases & -76.6 & 0.0 & 0.0 & 0.0 & -0.1 & -0.6 & -1.0 & -1.7 & -2.5 \\
\hline Change in other gross official reserves (increase -) & -33.8 & -112.7 & -44.1 & 16.0 & 29.1 & 4.9 & -8.8 & -39.7 & -57.7 \\
\hline Change in other foreign liabilities (increase + ) & 23.8 & 1.5 & -24.3 & -0.6 & 0.0 & 0.0 & 0.0 & 0.0 & 0.0 \\
\hline Change in arrears (decrease -) & 0.0 & 0.0 & 0.0 & 0.0 & 0.0 & 0.0 & 0.0 & 0.0 & 0.0 \\
\hline Exceptional financing & 0.0 & 0.0 & 0.0 & 0.0 & 0.0 & 0.0 & 0.0 & 0.0 & 0.0 \\
\hline Financing need & 0.0 & 0.0 & 0.0 & 1.7 & 0.0 & 0.0 & 0.0 & 0.0 & 0.0 \\
\hline Memorandum items: & & & & & & & & & \\
\hline Current account deficit (perce & & & & & & & & & \\
\hline Excluding official transfers & -13.5 & -13.1 & -15.5 & -17.8 & -16.8 & -15.3 & -14.1 & -13.3 & -12.4 \\
\hline Including official transfers & -4.7 & -2.4 & -5.5 & -6.6 & -9.5 & -9.0 & -8.5 & -8.0 & -7.3 \\
\hline Gross official reserves & 439.6 & 552.4 & 596.4 & 580.4 & 551.3 & 546.4 & 555.2 & 594.8 & 652.5 \\
\hline Gross official reserves (months of imports of G\&NFS) & 5.6 & 4.8 & 5.3 & 4.8 & 4.4 & 4.0 & 3.8 & 3.9 & 4.0 \\
\hline $\begin{array}{l}\text { Gross official reserves (including SDR allocation; months } \\
\text { of imports of G\&NFS) }\end{array}$ & 5.6 & 4.8 & 5.3 & 5.5 & 5.0 & 4.6 & 4.4 & 4.4 & 4.4 \\
\hline Overall balance (percent of GDP) & 2.9 & 3.2 & 1.5 & -0.4 & -0.5 & -0.1 & 0.2 & 0.6 & 0.8 \\
\hline Total external debt ${ }^{7}$ & 485.6 & 573.7 & 677.7 & 837.1 & 1015.0 & 1173.4 & 1367.2 & 1575.7 & 1793.9 \\
\hline Total external debt (percent of GDP) & 17.1 & 16.8 & 15.2 & 16.7 & 19.2 & 20.6 & 22.2 & 23.6 & 24.9 \\
\hline Debt service ratio (percent of exports) & 2.9 & 2.4 & 1.1 & 2.9 & 2.6 & 3.3 & 3.5 & 3.5 & 3.3 \\
\hline
\end{tabular}

Sources: Rwandan authorities; and IMF staff estimates and projections.

${ }^{1}$ Revision of methodology resulted in sharp increase of tourism revenues from 2008, thus increasing export of services.

${ }^{2}$ Including interest due to the Fund.

${ }^{3}$ Current transfers include disbursed budgetary and HIPC grants and humanitarian and technical assistance.

${ }^{4}$ Includes project and budgetary loans.

${ }^{\circ}$ Excluding payments to the Fund.

${ }^{6}$ Other capital includes long-term private capital, commercial credit, change in the net foreign assets of commercial banks, and unrecorded imports.

${ }^{7}$ After rescheduling, including arrears and new debt. 
Table 5. Rwanda: Financial Soundness Indicators for the Banking Sector, 2006-March 2009 (Ratios, in percent)

\begin{tabular}{|c|c|c|c|c|c|}
\hline Financial soundness indicators & 2006 & 2007 & $\begin{array}{c}\text { Jun } \\
2008\end{array}$ & $\begin{array}{c}\text { Dec } \\
2008\end{array}$ & $\begin{array}{c}\text { Mar } \\
2009\end{array}$ \\
\hline \multicolumn{6}{|l|}{ Capital adequacy } \\
\hline Solvency ratio & 13.7 & 16.6 & 16.6 & 15.9 & 19.1 \\
\hline Off balance sheet items/Total qualifying capital & 334.8 & 305.6 & 103.8 & 210.2 & 182.6 \\
\hline Insider loans/Core capital & 45.2 & 57.9 & 9.7 & 18.2 & 9.2 \\
\hline Large exposures/Core capital & 131.6 & 127.7 & 82.3 & 80.2 & 92.7 \\
\hline \multicolumn{6}{|l|}{ Asset quality } \\
\hline NPL's/Gross loans & 25.0 & 18.1 & 12.0 & 12.6 & 13.9 \\
\hline Provisions/NPL's & 83.5 & 67.0 & 75.5 & 66.3 & 66.9 \\
\hline Earning assets/Total assets & 85.3 & 59.6 & 80.9 & 82.4 & 78.3 \\
\hline Large exposures/Gross loans & 20.2 & 21.2 & 15.3 & 13.5 & 18.4 \\
\hline \multicolumn{6}{|l|}{ Profitability and earnings } \\
\hline Return on assets & 2.4 & 1.5 & 3.0 & 2.3 & 1.7 \\
\hline Return on equity & 27.0 & 15.5 & 24.4 & 18.0 & 11.8 \\
\hline Net interest margin & 8.0 & 5.2 & 10.1 & 9.8 & 10.2 \\
\hline Cost of deposits & 2.4 & 2.4 & 0.9 & 1.8 & 1.7 \\
\hline Cost to income & 76.7 & 80.2 & 61.0 & 77.8 & 83.7 \\
\hline Overhead to income & 46.2 & 44.2 & 38.6 & 48.8 & 43.7 \\
\hline \multicolumn{6}{|l|}{ Liquidity } \\
\hline Short-term gap & 34.2 & 21.5 & 25.5 & 21.3 & 18.3 \\
\hline Liquid assets/Total deposits & 64.7 & 76.4 & 60.4 & 61.1 & 64.4 \\
\hline Interbank borrowings/Total deposits & 11.3 & 10.3 & 5.7 & 8.1 & 9.3 \\
\hline NBR borrowings/Total deposits & 0.3 & 0.2 & 0.1 & 0.2 & 0.1 \\
\hline Gross loans/Total deposits & 71.1 & 63.3 & 85.2 & 99.5 & 88.9 \\
\hline \multicolumn{6}{|l|}{ Market sensitivity } \\
\hline Forex exposure/Core capital & 9.0 & 38.6 & 57.2 & 46.7 & 0.5 \\
\hline Forex loans/Forex deposits & 0.8 & 1.0 & 0.5 & 0.5 & 0.4 \\
\hline Forex assets/Forex liabilities & 104.4 & 112.0 & 105.4 & 102.5 & 93.5 \\
\hline
\end{tabular}

Source: National Bank of Rwanda. 
Table 6. Rwanda: Millennium Development Goals

\begin{tabular}{|c|c|c|c|c|}
\hline & 1990 & 1995 & 2000 & 2007 \\
\hline \multicolumn{5}{|l|}{ Goal 1: Eradicate extreme poverty and hunger } \\
\hline Employment to population ratio, $15+$, total $(\%)$ & 87 & 87 & 84 & 80 \\
\hline Employment to population ratio, ages $15-24$, total (\%) & 79 & 80 & 73 & 64 \\
\hline Income share held by lowest $20 \%$ & .. & .. & 5.3 & .. \\
\hline Malnutrition prevalence, weight for age ( $\%$ of children under 5 ) & 24.3 & .. & 20.3 & 18.0 \\
\hline Poverty headcount ratio at national poverty line (\% of population) & - & 51.2 & 60.3 & 56.9 \\
\hline Prevalence of undernourishment (\% of population) & 45 & 56 & .. & 40 \\
\hline Vulnerable employment, total (\% of total employment) &.. & 93 & .. & .. \\
\hline \multicolumn{5}{|l|}{ Goal 2: Achieve universal primary education } \\
\hline Literacy rate, youth female (\% of females ages $15-24)$ & .. & .. & 77 & .. \\
\hline Literacy rate, youth male (\% of males ages $15-24$ ) & .. &.. & 79 & .. \\
\hline Persistence to last grade of primary, total (\% of cohort) & & .. & 27 & .. \\
\hline Primary completion rate, total ( $\%$ of relevant age group) & 37 & .. & 21 & \\
\hline Total enrollment, primary (\% net) &.. &.. & 69 & 94 \\
\hline \multicolumn{5}{|l|}{ Goal 3: Promote gender equality and empower women } \\
\hline Proportion of seats held by women in national parliament (\%) & 17 & 17 & 17 & 49 \\
\hline Ratio of female to male enrollments in tertiary education & .. & .. & 44 & 62 \\
\hline Ratio of female to male primary enrollment & 92 & .. & 97 & 102 \\
\hline Ratio of female to male secondary enrollment & 73 & .. & 94 & 89 \\
\hline Ratio of young literate females to males (\% ages $15-24)$ & .. & & 98 &.. \\
\hline Share of women employed in the nonagricultural sector (\% of total nonagricultural employment) &.. & 32.4 & 33.0 & .. \\
\hline \multicolumn{5}{|l|}{ Goal 4: Reduce child mortality } \\
\hline Immunization, measles (\% of children ages $12-23$ months) & 83 & 84 & 74 & 99 \\
\hline Mortality rate, infant (per 1,000 live births) & 117 & 115 & 113 & 109 \\
\hline Mortality rate, under-5 (per 1,000$)$ & 195 & 193 & 189 & 181 \\
\hline \multicolumn{5}{|l|}{ Goal 5: Improve maternal health } \\
\hline Adolescent fertility rate (births per 1,000 women ages $15-19$ ) & .. & 58 & 50 & 40 \\
\hline Births attended by skilled health staff ( $\%$ of total) & 26 & .. & 31 & 39 \\
\hline Contraceptive prevalence ( $\%$ of women ages $15-49$ ) & 21 & 14 & 13 & 17 \\
\hline Maternal mortality ratio (modeled estimate, per 100,000 live births) & & .. & .. & 1,300 \\
\hline Pregnant women receiving prenatal care $(\%)$ & 94 & .. & 92 & 94 \\
\hline Unmet need for contraception (\% of married women ages 15-49) & 39 & .. & 36 & 38 \\
\hline \multicolumn{5}{|l|}{ Goal 6: Combat HIVIAIDS, malaria, and other diseases } \\
\hline Children with fever receiving antimalarial drugs ( $\%$ of children under age 5 with fever) &.. & .. & 13 & 12 \\
\hline Condom use, population ages $15-24$, female ( $\%$ of females ages $15-24$ ) & .. & .. & 3 & 5 \\
\hline Condom use, population ages $15-24$, male (\% of males ages $15-24)$ & $\cdot$ & .. & 24 & 19 \\
\hline Incidence of tuberculosis (per 100,000 people) & 167 & $24 \ddot{1}$ & 348 & 397 \\
\hline Prevalence of HIV, female (\% ages $15-24)$ & & & & 1.4 \\
\hline Prevalence of HIV, total (\% of population ages $15-49$ ) & 9.2 & 7.0 & 4.7 & 2.8 \\
\hline Tuberculosis cases detected under DOTS $(\%)$ &.. & 34 & 32 & 25 \\
\hline \multicolumn{5}{|l|}{ Goal 7: Ensure environmental sustainability } \\
\hline Annual freshwater withdrawals, total (\% of internal resources) &.. & .. & 1.6 & .. \\
\hline CO2 emissions (kg per PPP \$ of GDP) & 0.1 & $0 . \ddot{2}$ & 0.1 & 0.1 \\
\hline $\mathrm{CO} 2$ emissions (metric tons per capita) & 0.1 & 0.1 & 0.1 & 0.1 \\
\hline Forest area ( $\%$ of land area) & 13 & 13 & 14 & 19 \\
\hline Improved sanitation facilities (\% of population with access) & 29 & 26 & 25 & 23 \\
\hline Improved water source (\% of population with access) & 65 & 64 & 65 & 65 \\
\hline Marine protected areas, ( $\%$ of surface area) & .. & .. & .. & .. \\
\hline Nationally protected areas (\% of total land area) &.. & .. & .. & 8.1 \\
\hline \multicolumn{5}{|l|}{ Goal 8: Develop a global partnership for development } \\
\hline Aid per capita (current US $\$$ ) & 39 & 123 & 39 & 73 \\
\hline Debt service (PPG and IMF only, \% of exports of G\&S, excl. workers' remittances) & 10.0 & 20.0 & 25.0 & 3.0 \\
\hline Internet users (per 100 people) & 0.0 & 0.0 & 0.1 & 1.1 \\
\hline Mobile phone subscribers (per 100 people) & 0.0 & 0.0 & 0.5 & 7.0 \\
\hline Telephone mainlines (per 100 people) & 0.0 & 0.0 & 0.0 & 0.0 \\
\hline \multicolumn{5}{|l|}{ Other } \\
\hline Fertility rate, total (births per woman) & 7.4 & 6.4 & 6.0 & 5.9 \\
\hline GNI per capita, Atlas method (current US\$) & 350 & 220 & 240 & 320 \\
\hline GNI, Atlas method (current US\$) (billions) & 2.5 & 1.3 & 2.0 & 3.1 \\
\hline Gross capital formation (\% of GDP) & 14.6 & 13.4 & 18.3 & 21.2 \\
\hline Life expectancy at birth, total (years) & 32 & 31 & 40 & 46 \\
\hline Literacy rate, adult total (\% of people ages 15 and above) & 58 & & 65 & \\
\hline Population, total (millions) & 7.3 & $5 . \ddot{6}$ & 8.2 & $9 . \ddot{7}$ \\
\hline Trade (\% of GDP) & 19.7 & 31.0 & 34.4 & 38.2 \\
\hline
\end{tabular}

Source: World Development Indicators database.

Figures in italics refer to periods other than those specified. 
Table 7. Rwanda: Proposed Schedule of Disbursements Under the PRGF Arrangement, 2009

Date

Conditions necessary for disbursement

Amount

(In millions of (In percent SDRs)

1.17

1.46

August 5, 2009 Observance of quantitative performance criteria for December 31, 2008, completion of the sixth review under the arrangement. 


\section{Appendix I. Letter of Intent}

Kigali, July 23, 2009

Mr. Dominique Strauss-Kahn

Managing Director

International Monetary Fund

Washington, D.C. 20431

USA

Dear Mr. Strauss-Kahn:

1. The fifth review of our financial and economic program support under the Fund's Poverty Reduction and Growth Facility (PRGF) was completed on January 12, 2009. In the attached Memorandum of Economic and Financial Policies (MEFP) we review recent economic developments and progress in the implementation of our program through the first half of 2009, and set out policies for the medium term.

2. Program implementation under the PRGF arrangement has been broadly on track. All performance criteria for end-December 2008 were met. The indicative target on the net present value of external debt was not met, reflecting higher absorptive capacity of projects, which accelerated disbursement of existing loans. Due to severe external shocks affecting Rwanda, several indicative targets for end-March 2009 were missed.

3. Rwanda is affected by the global crisis, and its growth rate will slow from 11.2 percent in 2008 to an expected 5.3 percent in 2009. Falling demand in international markets and related decline in world prices will adversely affect our balance of payment. Our macroeconomic framework has been adjusted to reflect this impact.

4. To proceed with the rehabilitation and financing of Kigali's urban road network, the Government of Rwanda has contracted a nonconcessional external loan and requests a waiver for the continuous performance criterion on contracting of nonconcessional debt. This project is important to address our infrastructure and urban development needs.

5. The Government of Rwanda also requests a waiver for the continuous performance criterion on nonintroduction of multiple currency practice (MCP). In March, the National Bank of Rwanda introduced a foreign exchange risk hedging facility for the commercial banks in order to encourage foreign borrowing and longer-term domestic lending. As the facility has created an MCP, it was removed in June, before it was used. 
6. The Government of Rwanda has the intention to seek a new program with the IMF under the Policy Support Instrument (PSI) as a follow-up program to the PRGF arrangement. We would like to hold discussions on the new program in early 2010 at the time of the consideration of the budget for the new fiscal year July 2010/June 2011.

7. In the meantime, we have developed a medium-term macroeconomic framework with indicative quantitative targets and policy plans. A detailed macroeconomic program was also developed for June 2009 to July 2010. This will help bridge the gap between the end of economic program supported by the PRGF arrangement and the beginning of the successor program.

8. In support of our policies described in the MEFP, the Government of Rwanda requests completion of the sixth review under the PRGF arrangement and disbursement of the seventh loan of SDR 1.17 million.

9. The Government of Rwanda believes that the policies set forth in the attached MEFP are adequate to achieve the objectives of its program but will take any further measures that may become appropriate for this purpose. The Government of Rwanda will consult with the Fund on the adoption of these measures and in advance of revisions to the policies contained in the MEFP, in accordance with the Fund's policies on such consultations.

10. The Government of Rwanda authorizes the publication and distribution of this letter and MEFP together with the related staff report.

\author{
Sincerely yours, \\ $/ \mathrm{s} /$ \\ François Kanimba \\ Governor \\ National Bank of Rwanda \\ $/ \mathrm{s} /$ \\ James Musoni \\ Minister of Finance \\ and Economic Planning
}

Attachment: Memorandum of Economic and Financial Policies 


\section{Appendix I-Attachment 1:}

\section{Memorandum of Economic and Financial Polices (MEFP) of the Government of Rwanda}

\section{July 23, 2009}

1. The Government of Rwanda remains committed to achieving sustained economic growth and poverty reduction through the pursuit of prudent economic and social policies as well as structural reforms. The strategies to achieve these goals are set out in the Economic Development and Poverty Reduction Strategy (EDPRS) as well as in the Vision 2020 development plan. This memorandum of economic and financial policies (MEFP) reviews performance under the program supported by the IMF's Poverty Reduction and Growth Facility (PRGF) and describes the policies and targets for the medium-term as well as for the fiscal year July 2009/June 2010. The Government of Rwanda has the intention to seek a program with the IMF under the Policy Support Instrument (PSI) as a follow-up program to the PRGF. We would like to hold discussions on the new program in early-2010 at the time of the consideration of the budget for the new fiscal year July 2010/June 2011.

\section{Recent Economic Development}

2. Growth was strong in 2008 and reached 11.2 percent. This is the first double-digit real GDP growth in the last five years, which was achieved despite the volatile international financial crisis. Agriculture led the way with a real growth of 15 percent. This sector benefited from the favorable weather conditions as well as Government's crops intensification program supported with fertilizer application, improved seeds and storage facilities.

3. Despite monetary tightening, inflation accelerated in 2008. Rising prices of food, transport and housing and utilities pushed inflation to 22.3 percent by end-December. The average annual inflation was 15.4 percent. These developments partly reflect the passthrough of the high international food and fuel prices that also affected domestic food prices.

4. Fiscal performance was also strong. Total revenue collections at 15.6 percent of GDP were higher than programmed in 2008 because of higher growth, inflation, increased monetization of the economy and "one off" payments. Tax revenue collections were 19.5 percent higher than programmed and reached 13.5 percent of GDP benefiting from the collection of tax arrears of RWANDATEL. Non-tax collections that contributed about 2.1 percent of GDP to revenues were RWF 30 billion higher than programmed on account of "one off" accrual of RWF33.4 billion (US\$60 million, 1.4 percent of GDP) in mobile license fees from TIGO company. 
5. Government spending on priority programs accelerated during the second half of the year because of the purchases of seeds and fertilizer and spending on storage facilities (largely financed by grants from IDA and AfDB) and maintenance of the road network. The accrual of the additional revenue during the year allowed the Government to increase outlays for various energy, water and sanitation projects in line with the EDPRS.

6. All end-2008 fiscal program targets were met. In the case of the targets for net credit to Government (NCG), domestic fiscal balance (DFB) and priority spending, these were met with comfortable margins largely on account of the "one-off "receipts. The net liquidity impact of fiscal operations, given the high import content of the budget, amounted to 2.7 percent of GDP in 2008, lower than envisaged in the program.

7. The external current account deteriorated sharply compared to 2007. Despite a strong increase in export receipts from coffee, tea, minerals and tourism, the trade deficit widened because of rising import volumes and a surge in food and fuel prices. As a result the terms of trade also deteriorated sharply in 2008. The accrual of donor disbursements together with the inflow of the fees for the mobile license by TIGO led to an increase in gross official reserves from 4.8 months at end-2007 to 5.3 months by end-2008.

8. Regarding the exchange rate policy, over 2008 the Rwandan Franc depreciated by almost 2 percent against the U.S. dollar; however due to the high inflation in 2008, the Rwandan appreciated significantly in real terms against the dollar during the year. It is worth noting that more foreign exchange was sold in 2008 than in 2007, partly reflecting the high demand for imports. However, the stock of foreign exchange has increased over the year as a result of donor disbursements made during 2008 and the large sale of the telecommunication license.

9. The end-December 2008 targets for reserve money and Net Foreign Assets (NFA) were met. However, there was a persistence of high levels of currency in circulation and vault cash, which increased in the face of rapid pace of economic growth, inflationary developments and the opening of new bank branch networks. NBR relied more on the sale of foreign exchange for sterilization purposes rather than on the issuance of domestic instruments. As a result, domestic debt declined sharply in 2008. This policy was also in response to the demand of both the private sector and Government for foreign exchange to finance the high cost of imports in 2008.

10. Broad money growth declined to 5.5 percent at end-2008, which was well below the program projections. The broad money even began to decrease in nominal terms from November 2008 due to falling deposits. The decline in deposits was mainly the result of some large depositors withdrawing their deposits for long term investment both locally and externally. By end-2008 there was a tightening of the liquidity in the banking system. The 
Central Bank responded to this situation by (i) reducing the reserve requirement ratio by 3 percentage points from 8 percent to 5 percent in early 2009 , as well as significantly reducing the stock of outstanding domestic assets (including treasury bills and repos).

\section{Structural Reforms}

11. Significant progress has been made in developing Rwanda's financial sector. The number of people accessing formal financial services increased. In line with the objective of the government to increase access to financial services to the population, in 2008 the Central Bank licensed two more commercial banks. These are Banque Populaire du Rwanda which was upgraded from a microfinance institution and the Kenya Commercial Bank Rwanda. In addition, NBR permitted the establishment of 23 new microfinance institutions. Furthermore, six new commercial bank branches were opened in 2008. The total number of beneficiaries of formal financial services has increased by 29.5 percent from 2007 to $2008 .{ }^{1}$ Structural reforms continued to be developed across the sector, with notable achievements including:

- $\quad$ Amendments made to the Central Bank Act. These amendments, made in February 2008, gave NBR the authority to regulate the non-banking financial sector, comprising insurance companies, brokers and pension funds. The Central Bank will be able to draw from its experience in regulating the banking sector to ensure the safety and soundness of the non bank financial sector.

- $\quad$ A number of systems have been implemented by NBR under the new National Payments Strategy. As planned, the switch for card-based payments is operational. A number of key supporting policies are close to being ready for full implementation, including the operation of the Automated Clearing House and the Real Time Gross Settlement system (integrated with the Central Securities Depository). In addition, a National Payments Council has been established to oversee and advise this process, which involves representation from the commercial banks, MFIs, Government, teams involved in the national switch-over, telecoms representatives and the Rwandan Development Board.

- $\quad$ The Insurance Sector Law came into force in March 2009. The core set of implementing regulations have been released for comments by the industry and will be issued as soon as these comments are discussed and evaluated.

- $\quad$ In line with the license issued in 2008, a private credit reference bureau will be operational before the end of 2009. The private credit bureau will replace the public 
credit bureau housed at the Central Bank and will now also cover microfinance institutions. The purpose of the new bureau will be to compile information concerning the repayment behaviour of individuals and businesses and will organize this information for resale to banks, credit providers, insurance companies and other eligible entities. The information provided by credit bureaus is used by credit providers to better assess the risks posed by a particular credit application, based on the credit customer's ability to manage credit in the past. Credit Bureaus are an essential component in the development of a sound financial sector and a robust credit industry.

12. Progress has been made in reforming Public Financial Management (PFM) in 2008 and early 2009.

- $\quad$ The PFM Reform Strategy (2008-2012) was approved by Cabinet in December 2008. Since the strategy was approved, a PFM steering committee has been established and the first meetings were held in early 2009. To further embed the principles of PFM, a Manual of Budget Processes and Procedures has recently been approved and training on the content has been scheduled for July 2009. In addition, the piloting of an Integrated Personnel and Payroll Information System has been successfully managed by MIFOTRA, with civil servant salaries paid through this route since February 2009. Further pilots are due across a number of other Ministries over 2009, streamlining payment processes.

- $\quad$ Embedding the principles of MTEF at every level of government. The government's MTEF manual formalized practices already in use across the budget process. Indeed, the practices outlined in this document were central to formulating the mini-budget for 2009 as well as the budget for the new financial year 2009/10 that was recently approved by the Parliament. To ensure complete implementation of the manual across all budget agencies, MINECOFIN is currently recruiting a consultant to undertake comprehensive training of all planning and budgeting officers to reinforce the MTEF principles. An appointment will be made by the end of June 2009, with training to commence shortly after.

- $\quad$ The Government and development partners signed a memorandum for budget support harmonization. Sector-Wide Approaches (SWAPs), designed to harmonize and align donor interventions, were adopted in the energy, transport and agriculture sectors.

\footnotetext{
${ }^{1}$ Beneficiaries of all formal financial services (including microfinance institutions) increased from 1.4 million in 2007 to 1.8 million in 2008 .
} 


\section{Program Performance in the First Half of 2009}

13. Multiple external and domestic shocks affected economic performance during the first half of 2009. These shocks are expected to reduce demand for exports, especially minerals, reduce income and revenues from tourism and remittances, as well as foreign direct investments. Despite these shocks, performance at end-March 2009 was satisfactory as inflation decelerated on account of good season A harvest.

14. Two indicative fiscal targets for end-March were missed. The targets for Net Credit to Government (NCG) and the Domestic Fiscal Balance (DFB) were missed by some margins. These targets were missed mostly because of the delayed disbursement of budget support grants from the World Bank and the need to finance the DRC military operations, as well as the frontloading of some priority expenditures. The Government financed these expenditures by drawing down deposits in NBR. The target spending for priority expenditures was met with a significant margin.

15. The indicative target for net repayment of domestic arrears was also missed at endMarch. This reflected delays in implementing new payment procedures to reduce frauds and increase transparency in payments. Where payments orders did not meet all legal requirements, these were cancelled and re-issued subsequently. Moreover currently, there is lack of synchronization between the payment information system of MINECOFIN and NBR as this is done manually, resulting in a perceived accumulation of arrears though payments by NBR have been effected. Efforts are ongoing to provide an electronic interface between the two softwares (using SmartGov). This will allow automatic synchronized recording of all payments. In the second quarter, there was a sharp reduction of arrears as payments accelerated; as a result end-June target is likely to be met with a margin.

16. Revenue collection during the first quarter matched projections despite the impact of the world economic crisis. Tax and customs revenue collections exceeded projections reflecting a still robust economic growth at the end of 2008 and delayed high imports in early 2009. These imports contained a large portion of capital goods that were partly funded by a drawdown of Government deposits.

17. Government spending exceeded program projections by about RWF 12 billion during the first quarter on account of the internationally approved military operations in the DRC at the beginning of the year, as well as the frontloading of spending on telecommunications, water and energy projects.

18. The impact of the external shocks were felt in the early months of 2009 as exports declined by about 10 percent in U.S. dollar value terms in the first quarter compared to the same period last year. On the other hand, imports in value terms were about 58 percent higher than in the corresponding period of 2008. This reflected delays in arrival of imports 
contracted last year at high prices as well as a still robust economic growth at end-2008. As a result the trade deficit widened significantly compared to the same period last year.

19. The Net Foreign Assets (NFA) of NBR declined sharply by about 22percent during the first quarter of 2009. This reflected the delayed disbursements of budget support grants from the World Bank and the need to sell foreign exchange to support import activities. The end-March NFA indicative target was thus missed. Broad money also declined by about 8 percent during the same period, reflecting a decline in currency in circulation and deposits. These developments also reflected the tight liquidity situation in the first quarter of 2009.

20. Regarding fiscal performance in the first half of 2009, the continuing economic growth, high imports and improvements in Rwanda Revenue Authority's (RRA) efficiency will likely keep revenue collections at the level projected for this period. Strict adherence to expenditure allocation levels as well as enforcement of spending procedures are expected to reduce the incidence of over-spending as well as avoid arrears build up by ministries and agencies. According to our preliminary information, Net Credit to Government and Domestic Fiscal Balance indicative targets for end-June 2009 are likely to be missed on account of (i) delays of disbursement of sector budget support grants, and (ii) expenditures related to the military operations in DRC, which were not foreseen when program targets were set. The Priority Expenditure target was likely met with a comfortable margin.

\section{Macroeconomic Outlook and Medium Term Strategy}

21. Multiple external and domestic shocks are expected to continue to impact on economic performance for the rest of the year as well as for the medium term, in particular:

- $\quad$ Real GDP growth will be led by the agriculture sector, which will continue to benefit from investment in inputs and extension services. This is evident in the strong harvest observed in season A. However, growth in the medium term is expected to be lower than the exceptional performance in 2008/09.

- The global economic and financial crisis will impact negatively on economic performance by reducing financial inflows through two main channels; (i) declining external demand for Rwandan goods and services with tourism and minerals strongly hit and (ii) reducing other financial inflows including remittances and FDIs.

- $\quad$ The current liquidity shortage could exacerbate the impact of the global financial crisis by reducing the availability of domestic credit for growth. The stock of credit to the private sector had already declined by 0.5 percent during the first four months of 2009. 
22. In light of these factors, real GDP growth is projected to slow down in 2009-10. Real GDP growth is expected to decline from the estimated 11.2 percent in 2008 to about 5.5 percent in 2009/2010 reflecting the slowdown of non agricultural growth. Construction and real estate, mining hotel and restaurants and trade will be particularly affected by the global crisis and the liquidity shortage. From 2010, however, a gradual recovery of the nonagricultural sector in line with developments in the world economy is expected. This together with a normal agricultural growth will be expected to raise real GDP growth to about 6.3 percent in 2010/11 and about 7 percent in subsequent years.

23. Inflation is projected to decline to single digits in the second half of 2009. This decline will result largely from a reduction in import prices, lower domestic food prices on account of the good harvest and an easing of domestic demand pressures. The headline inflation has already fallen from 22 percent at end-December 2008 to about 12.7 percent at end-May, largely reflecting slowing growth of food and housing prices. Continued prudent monetary management will be required to keep inflationary expectations down so as to achieve the target inflation rate of 5 percent in 2010.

24. The balance of payments is likely to deteriorate in 2009-2010. This reflects the sharp deterioration in the external environment as exports of goods and services are expected to decrease by about 23 percent in U.S. dollar terms in 2009 compared to 2008. This decline will be partially offset by a reduction of approximately 7 percent in imports. Despite frontloading of official foreign assistance, the current account deficit (including official transfers) is expected to deteriorate slightly from 5.5 percent of GDP in 2008 to about 6.6 percent of GDP in 2009. Foreign Direct Investment and other capital inflows are also expected to fall, resulting in the deterioration of the overall balance of payments by about US\$ 80 million compared to 2008.

25. Although exports of goods and services and other private capital flows are expected to recover from 2010 as the global environment improves, the concomitant increase in imports to support the EDPRS investment needs and the projected decline in official transfers would be expected to lead to a further worsening of the balance of payments. An overall deficit of about US\$ 30 million is projected for 2010. To support the projected import values in line with EDPRS investment needs, some draw down of international reserves can be accommodated in the next few years. The balance of payments is expected to improve from 2011 as the world economy recovers and demand for Rwandan goods and services increases. However, the current external shock could have a lasting impact on the reserve coverage of the country, which could stabilize at about 4 months of imports over the medium term, down from about 5.3 months at end-December 2008.

26. The medium-term balance of payments projections assume greater exchange rate flexibility to stem the loss of foreign exchange reserves. This will help gradually offset the competitiveness losses that Rwanda suffered as a result of the large real appreciation in 2008. 
27. Fiscal policy will have to balance the competing objectives of cushioning the impact of the economic crisis on growth and poverty reduction as envisaged in the EDPRS whilst preserving the medium-term fiscal and external sustainability. Declining revenues, and from 2010/11 lower committed external budget support, are expected to reduce the available budget resources. For fiscal year 2009/10, fiscal revenues are expected to decline by about 2.4 percent of GDP compared to 2008/09. This largely reflects absence of "one off" payments, slowing economic activity, losses from adopting the East African Community (EAC) common external tariff (CET), and elimination of value added tax on large trucks and surcharge on sugar.

28. In the medium-term, revenues are projected to increase by at least 0.2 percent of GDP per year, reflecting both economic recovery and continued increases in efficiency of tax collection. Grant financing is projected to remain high in 2009/10, but the projection for 2010/12 takes into account only the grants already committed by donors. This shows a decline of about 2-3 percent of GDP compared to the level of 2009/10. To maintain expenditures at a level of the 2009/10 as a share of GDP, projections envisage an increase in concessional borrowing.

29. The expenditure plan shows a gradual adjustment partly offsetting the reduction in resources. The total expenditure in the budget for fiscal year 2009/10 approved by Parliament amounted to 26.9 percent of GDP. A gradual reduction to about 25.2 percent of GDP by $2011 / 12$ is envisaged. The main source of the reduction is externally financed capital expenditure, which reflects expected reduction in donor grants for capital projects. Recurrent expenditure and domestically financed capital expenditure for 2009/10 are projected at about 20.2 percent of GDP, confirming that the expenditure profile will be sufficient to achieve the objectives outlined in Rwanda's poverty reduction strategy (EDPRS). Priority expenditures will remain at about 13 percent of GDP during this period.

30. The budget deficit (excluding grants) is expected to rise sharply to 13.6 percent of GDP in the 2009/10 budget but thereafter decline to about 11.5 percent of GDP by 2011/12. The increase in 2009/10 and the subsequent decline are broadly consistent with the macroeconomic policy objectives and available financing. The projected net liquidity impact of the budget spending (about 2 percent of GDP per year in 2009/10-2011/12) will provide a stimulus to the economy without compromising the Government's objective of restraining inflation to single digits. The expanding budget deficit in 2009/10 would imply a draw down of government deposits in the NBR of up to 1 percent of GDP. This would be covered by funds from the mobile telephone license fees of about 1.4 percent of GDP received at the end of 2008. Consistent with the medium-term projections, the budget does not envisage any further domestic bank financing except for monetary policy purposes. An update of the debt sustainability analysis (DSA) indicates that the medium-term fiscal profile is broadly consistent with the maintenance of public debt at a sustainable level. 
31. The Government is in the process of studying the macroeconomic implications of its plans for the pension system reform and the introduction of the Provident Fund. This will involve a possible redirection of a part of central Government revenue (and expenditure) to the Provident Fund. Given that more time is needed for the Government to assess the implications of the possible reforms for the central budget, including the revenue losses and offsetting measures for them, the budget for 2009/10 does not include these reforms, and the current medium term projections do not yet include the estimates of the impact. These will be included in the fiscal framework once the technical work has been completed.

32. Monetary policy in the medium term will be more proactive in averting balance of payment pressures as well as moderating inflation. It will also pay more attention to managing the bank liquidity situation. The NBR will continue to use reserve money as the operational target to control inflation. To achieve this objective, NBR will aim to meet the reserve money target on average and not only at end-month, through a coordinated sale of domestic and foreign sterilization instruments. NBR will work with the Fund team to develop a reserve money operational framework that would reduce volatility and improve its effectiveness as an operational target.

\section{The Macroeconomic Program for Fiscal Year 2009/10}

33. The fiscal year 2009/10 marks the beginning of Rwanda's aligning itself to the EAC budget calendar as well as implementing the EAC Common External Tariff (CET) from $1^{\text {st }}$ July 2009 under the Customs Management Act. This implies that the customs tariff bands will change from tariff bands of 30 percent for finished products, 15 percent for semifinished goods, 5 percent and 0 percent for raw materials to a three tariff band of 25 percent for finished goods, 10 percent for semi finished goods and 0 percent for raw materials. Under the CET, taxes on international trade will be levied at point of entry basis instead of CIF Kigali.

34. Managing the impact of the external and internal shocks on economic performance will be at the heart of our macroeconomic policies. On the real GDP growth front, we will intensify our investment and extension service activities in agriculture as the lead sector to achieve our projected growth of 5.3 percent in 2009. The expected good harvest together with our fiscal and monetary policies that are outlined below should enable us to achieve single digit inflation in mid-2009.

35. Regarding fiscal policy, the domestic fiscal deficit (excluding spending on demobilization and peacekeeping operations) is projected to rise from about 5.3 percent in calendar year 2008 to about 6.2 percent in fiscal year 2009/10. This will result from increasing allocation of budgetary resources to investment and other priority projects outlined in the EDPRS, which will provide a solid base for growth in Rwanda. 
36. We expect fiscal revenues to decline by about 2.4 percent of GDP compared to 2008/09. This is due to reasons previously highlighted in paragraph 27 . These losses will be partially offset by additional revenue measures introduced by the Government, which include increasing fuel levy earmarked for road maintenance, gradually eliminating implicit subsidies on petroleum products and raising excise taxes on mobile airtime.

37. In the case of expenditures, the focus lies in increasing resources to public infrastructure, especially energy and roads. The development of physical infrastructure aims at reducing the cost of doing business in Rwanda to promote private sector led growth. In addition, more resources have been allocated to agriculture, education, health and water. Priority spending will remain high at about 13 percent of GDP, about the same level as in 2008. The priority areas are as follows:

- $\quad$ Agriculture - we will pursue a multi-pronged approach to growth in this sector. Activities will focus on the provision of fertilizer and improved seeds and other inputs. Funds for reduction of soil erosion, irrigation and water management as well as marshland development have been increased.

- $\quad$ Energy - to address the issue of limited and insufficient energy, we have allocated sufficient funds to continue various energy projects including the Nyabarongo hydro dam as well as other microdam projects. In addition, funds have been allocated for investment in the Lake Kivu gas project, provision of heavy fuel generating plant for Electrogaz as well as for rehabilitation of distribution infrastructure. It is expected that the implementation of all these projects will make it possible to reduce electricity tariff by half after 2010 as well as cut the subsidy to Electrogaz by 0.5 percent of GDP in 2010-12.

- $\quad$ Roads - we will undertake various projects aimed at upgrading and rehabilitating key road networks in the country including Kigali and the surroundings. With regards to the Kigali and surroundings road network, we have contracted a loan of US $\$ 33$ million from the ExImBank of China at end-June 2009. This project is of utmost importance to urban and economic development. The World Bank has confirmed its technical and economic viability. Other more concessional financing for this project was not available. Development partners indicated that this would not jeopardize Rwanda's access to future concessional financing. This loan has a grant element of 34 percent and our analysis shows that the loan will not significantly worsen the external debt sustainability. We are therefore requesting a waiver for the continuous performance criterion on contracting nonconcessional debt.

- Information and communication technologies (ICT) - we will continue to invest the proceeds from the sale of Rwandatel in the ICT sector. The objective of this project is to provide a robust nationwide broadband backbone using fiber optics. Over time it is 
expected that the availability of this ICT backbone will provide a platform to induce new economic activity countrywide.

- $\quad$ Education and health - In line with our EDPRS policies, we will retain the momentum generated last year in these sectors. Regarding education, after achieving a significant increase in enrolment, the focus is now turned to improving quality of the "9 year basic education" by raising the completion rate and strengthening postbasic education. We will therefore intensify the rehabilitation of existing classrooms as well as construction of new ones. We will also speed up the distribution of textbooks to improve learning, intensify the training of teachers to improve standards as well as distribute 100,000 laptops to various schools to encourage distance learning and promote computer literacy. In the case of health, the budget will support activities aimed at improving human health and slowing down population growth. The focus will therefore be on the purchase of drugs, vaccines and other medical supplies as well as catering for the "mutuelle" insurance schemes and expanding the contractual approach to community health programs.

PRIORITY EXPENDITURE AS PERCENT OF GDP, 2008/09 AND 2009/10

2008/09

2009/10

EMPLOYEE COST

1.9

1.8

GOODS AND SERVICES

2.0

2.0

TRANSFER AND SUBSIDIES

4.0

4.5

EXCEPTIONAL EXPENDITURES

0.5

0.2

NET LENDING

0.1

0.1

DOMESTIC CAPITAL

4.5

4.5

Total

12.8

13.1

Memorandum item:

Total expenditure and net lending (excluding privatization receipts) as percent of GDP

27.8

26.7

- Water and sanitation - we will continue with the rehabilitation of existing water and sanitation infrastructure as well as construction of new ones to improve delivery of services. In the area of water facilities we will finalize the construction of water pipes to various districts and towns including the supply of Kigali with water from the Nyabarongo underground sources. To improve sanitation, we will also complete the construction of various schools and town latrines. 
38. On the external front, the impact of the global crisis will result in a slight widening of the current account deficit in the next fiscal year. We will therefore draw down a small amount of our international reserves to enable us sustain our projected level of imports to achieve our growth objective. Should the balance of payments situation unexpectedly deteriorate, to stem the loss of reserves beyond what we have projected we will allow greater flexibility in the exchange rate by replacing the current system of on-demand sales of foreign currency to commercial banks with an auction system based on predetermined foreign exchange sales.

39. Reserve money will remain the anchor of the monetary program for the fiscal year 2009/10 to return and maintain inflation at single digits. We will therefore limit reserve money growth in the July- December 2009 and the January-June 2010 periods to about 6.5 percent and 7.5 percent respectively. To create enough room for an expansion of private sector credit, NBR may have to inject medium to long term liquidity in the banking system.

40. We will implement the facility for collateralized three to twelve month lending by NBR consistent with the reserve money target. The interest rate on the scheme, currently at 11.5 percent, will be changed in line with future changes in policy interest rates.

41. We will further improve our liquidity forecasting and coordination of fiscal and monetary policies. In this regard, we will strengthen the Treasury Management Committee (TMC) to enable it improve the coordination of fiscal and monetary policies. To this end it will pay more attention to the monitoring of the import component and the domestic demand impact of fiscal spending.

42. To prevent the risk of sharp credit slowdown, the Government will temporarily move part of its deposits currently at the NBR to commercial banks. The scheme is currently assessed and limited to 12 billion Rwf for the second half of 2009. Banks that increase the stock of their investment lending with maturity of over 3 years will be eligible for the government deposits. Banks will select the projects, will bear the credit risk, and set the lending rate. At the same time, the participation in the scheme will be voluntary. Only commercial banks that meet the Capital Adequacy Ratio will be eligible to participate in the scheme.

43. The NBR will also aim to restore positive real interest rates to prevent further deterioration of private sector deposits in commercial banks.

44. The NBR will strengthen its efforts to improve the functioning of the banking system and bank supervision. The focus will be on the following areas:

- We will finalize and implement a new liquidity regulation which will require banks to comprehensively report on maturity of assets and liabilities. On the basis of these 
reports, we will develop a new and more comprehensive liquidity ratio. When necessary, we will require that banks prepare action plan, including contingency funding plans if needed, to maintain an adequate liquidity position.

- We will implement the recently approved regulation on classification of nonperforming loans and provisioning. In addition, the capital adequacy requirement was raised in May from 10 to 15 percent to further strengthen the banking sector.

- We will enforce better risk management practices and corporate governance in the commercial banks, in line with existing regulations. In particular, we will advise each commercial bank to present its risk management program to be discussed with NBR before end-2009.

- We will strengthen risk-based bank supervision. We will rebuild the Banking Supervision Department capacity to conduct on-site inspections once a year as soon as possible. We will hire, on a temporary basis, external auditors to assist banking supervision department at the NBR in undertaking on-site inspections of two large banks by end-2009.

- We will augment the supervisory capacity at the NBR. Therefore, we will address the staffing shortage at the bank supervision department. Recruiting and training of new staff is now underway. In addition, by end-2009, we will improve the salary structure of the NBR to enhance motivation of the staff.

45. On the upcoming SDR allocation, this will increase the Net International Reserve position of Rwanda. While this will provide an additional buffer to gross reserves, it will not impact Rwanda's Net Foreign Assets position because both foreign assets and liabilities will increase. Because Rwanda's reserve cover in our baseline scenario is projected to be reduced from 5.3 months of imports to about 4 months of imports in the medium term, and the proposed fiscal stance is appropriate, we will save the SDR reserves as a safeguard against potential balance of payments pressure in the medium term. Should the balance of payments deteriorate more than expected, the use of the SDR allocation will be revisited at that time.

\section{Structural Policies for Fiscal Year 2009/10}

46. The focus on the structural side will remain on enhancing the productivity of the agricultural and export sectors, improving conditions for the private sector and effectiveness of public financial management.

\section{Export Promotion}


- $\quad$ Our export promotion strategy will continue to focus on enhancing the productivity in traditional sectors and diversifying our export base. New developments include the formation of a National Export Board which is to be established in July 2009 and will incorporate promotion of all agricultural exports under one institution. In the tea sector, 2009/10 will see the privatization of three factories which is hoped to improve productivity. For coffee, a Marketing Alliance will be established in 2009/10 to improve marketing. On tourism, a new regional approach aims to market EAC as one destination with a diversification of activities within. Rwanda will continue to focus on high-end cultural and eco-tourism.

\section{Financial Sector}

- $\quad$ The Financial Sector Development Plan (FSDP) is expected to be fully implemented by June 2010. The multifaceted FSDP has the task of strengthening and deepening the financial sector in Rwanda from microfinance to long term capital markets. Areas still to be completed include:

- $\quad$ Improving access to credit. Regulations for the newly developed Savings and Credit Cooperatives (SACCOs) and Micro Finance Institutions are ready for gazetting, thereafter they will be implemented. A private credit reference bureau which has been be set up will commence work to improve the quality of information and the assessment of risk posed by banks credit customers.

- Savings. Implementation of the National Savings Mobilization Strategy and the Umurenge SACCO's strategy (adopted by Cabinet in early 2009) will commence soon. These strategies will aid the Government in reaching the gross national savings target of 10 percent of GDP by 2012 (8.5 percent in 2008/09).

- $\quad$ Improving the Pensions Sector. Liberalization of the pension sector will be achieved by setting up a legal framework for the private pension scheme and personal retirement saving accounts. The law is expected to be officially published in early 2010.

- $\quad$ Regulation and Legal Framework for the Insurance Industry. NBR will work on legal and structural issues in the coming year to ensure a well regulated insurance sector.

- $\quad$ Modernization of the National Payment System. The NPS framework and strategy among others encapsulates the vision and projects will be undertaken to continue to modernize the Rwandan Payment Systems. The aim of this strategy is to have a clear vision and plans that we will enable us to set up an efficient, safe, fast and reliable payment system and expedite high value funds transfer. Many stakeholders are 
involved as indicated in the membership of the National Payments Council (NPC) where there is a wide range of representation.

\section{Cost of Doing Business}

- Efforts to improve the business climate will continue. A key development will be the modernization of Rwanda's business registration. A registry for secured transactions and intellectual property rights will be established. Moreover, businesses will benefit from the developments in online taxation, as well as other online applications and issuance of certificates; for example, it is planned to make it possible to register a business on-line (by the end of 2009).

\section{Public Investment and Public Private Partnerships}

- $\quad$ The Government will utilize its new National Public Investment Policy to improve resource allocation, quality, and efficiency of the investment portfolio and increased level of project execution rates. In 2009/10 it will strengthen the newly established instruments for public investment. These include the Public Investment Committee and the Technical Team. In addition, we will finalize the institutional framework as well as the operational guidelines. Care will be taken to ensure alignment of public investment with the new Public Private Partnership program and Rwanda's development goals.

- To foster private sector participation in infrastructure development, while enhancing the government's capacity to manage related fiscal risks, the Government will develop a Public Private Partnership (PPP) framework. During the year, the Government shall develop and adopt an agreed PPP framework that will set the scope of PPP program, detailed institutional and legal framework, regulations and procedures, procurement and negotiation, and the appropriate distribution of risks between the public and private sectors. 


\section{Fiscal Structural Reforms}

- Tax administration reforms will continue to achieve our targets for widening the tax base. To achieve the goal we will focus on three areas in 2009/10:

- $\quad$ Enhancing taxpayer compliance. To widen the tax base we will effectively implement the Block Management System for which a pilot program has begun and a national roll out is expected by January 2010. Since employers will be forced to declare the same figures for PAYE tax and social security compliance levels are expected to rise.

- $\quad$ Increasing efficiency of operation and minimizing transaction costs. We are planning to increase the amount of transactions customers can carry out online. This will increase the use of banks for payments, and tax services will be decentralized away from headquarters and closer to businesses.

- Facilitating trade. Cargo scanners will be introduced by January 2010 to assist in expediting the clearance of cargo, and so reducing the need for timely physical verification of imported goods. We will continue to provide incentives to compliant taxpayers and enforce appropriate sanctions to the non compliant.

52. Specific tax reforms will be imposed to enhance the effectiveness of the decentralization process. The RRA will strengthen its presence in the provinces and computerize all provincial offices by the end of 2010. At the district level the Government will undertake a study to identify new revenue sources available at the local level. It will recommend possible policy actions to widen revenue base.

\section{Public Financial Management}

- The Government will undertake a range of measures in continuation of Public Financial Management (PFM) reforms. Some of the activities for 2009/10 include:

- $\quad$ The MTEF process will be revitalized and integrated fully into budget preparation focusing on strengthening performance based budgeting. The costing of all major sectors for MTEF purposes will continue in 2009/10. Gender budgeting and performance-based budgeting will be mainstreamed into the budget process. Furthermore, Government will ensure that all externally financed projects and programs which managed by Government are fully reflected in the budget in order to minimize extra-budgetary funding. 
- $\quad$ Strengthen the newly established Rwanda Public Procurement Authority (RPPA). A new organizational structure and human resources strategy will be adopted by the RPPA, which is linked with anti-corruption institutions.

- IT improvements - 'Smartgov'. The budget IT system will be developed to increase coverage of expenditures which are carried out outside the treasury system. This includes self-generated revenues of agencies and districts and donor-funded expenditures. Improvements also include the management of government bank accounts. This will assist the monitoring of project execution rates. The pilot for this new system should begin in July 2009.

- District level reforms. Pilot projects have begun in the installation of new software for Tax Management Systems for districts, it is expected that this can begin to be rolled out to districts by end-2009. Training of local government staff in financial management procedures and reporting, focusing on local revenue mobilization will be carried out. 
Att. 1. Table 1. Rwanda: Quantitative Performance Criteria and Benchmarks for 2008 (Billions of Rwandan francs, unless otherwise indicated) (Quantitative benchmarks* and performance criteria on test dates ${ }^{* *}$ )

\begin{tabular}{|c|c|c|c|c|}
\hline \multirow{3}{*}{ Benchmarks and performance criteria } & \multicolumn{4}{|c|}{2008} \\
\hline & \multirow[t]{2}{*}{ March* $^{*}$} & \multirow[t]{2}{*}{ June $e^{\star *}$} & \multirow[t]{2}{*}{ September* } & \multirow[t]{2}{*}{ December**$^{* *}$} \\
\hline & & & & \\
\hline \multicolumn{5}{|l|}{ Net foreign assets of the NBR (floor on stock) ${ }^{1}$} \\
\hline Actual (program exchange rate) & 297.0 & 331.9 & 315.8 & 317.5 \\
\hline Adjusted program & 257.5 & 321.8 & 301.8 & 272.9 \\
\hline Program & 257.5 & 321.8 & 314.8 & 275.7 \\
\hline \multicolumn{5}{|l|}{ Reserve money (ceiling on stock) ${ }^{2}$} \\
\hline Actual & 101.1 & 106.6 & 112.2 & 114.1 \\
\hline Adjusted program & 100.3 & 107.7 & 113.8 & 114.8 \\
\hline Program & 98.0 & 102.4 & 107.1 & 107.6 \\
\hline \multicolumn{5}{|l|}{ Net credit to the government (ceiling on flow) ${ }^{3}$} \\
\hline Actual & -29.0 & -62.1 & -33.7 & -59.4 \\
\hline Adjusted program & 47.5 & -46.9 & -22.4 & -10.7 \\
\hline Program & 47.5 & -46.8 & -35.4 & -13.4 \\
\hline \multicolumn{5}{|l|}{ Domestic fiscal balance (floor on flow) $)^{3,4}$} \\
\hline Actual & -30.2 & -48.9 & -100.1 & -128.1 \\
\hline Adjusted program & -42.4 & -67.8 & -108.3 & -148.6 \\
\hline Program & -42.4 & -67.8 & -113.7 & -148.6 \\
\hline \multicolumn{5}{|l|}{ Total priority spending (floor on flow) $)^{3,4}$} \\
\hline Actual & 63.3 & 129.5 & 220.7 & 313.1 \\
\hline Adjusted program & 57.3 & 128.4 & 199.0 & 275.1 \\
\hline Program & 57.3 & 128.4 & 201.7 & 275.1 \\
\hline \multicolumn{5}{|l|}{ New nonconcessional external debt ${ }^{5}$} \\
\hline Actual & 0.0 & 0.0 & 0.0 & 0.0 \\
\hline Program & 0.0 & 0.0 & 0.0 & 0.0 \\
\hline \multicolumn{5}{|l|}{ New external payment arrears (ceiling on stock) 5, 6} \\
\hline Actual & 0.0 & 0.0 & 0.0 & 0.0 \\
\hline Program & 0.0 & 0.0 & 0.0 & 0.0 \\
\hline \multicolumn{5}{|l|}{ Short-term external debt (ceiling on stock) ${ }^{7}$} \\
\hline Actual & 0.0 & 0.0 & 0.0 & 0.0 \\
\hline Program & 0.0 & 0.0 & 0.0 & 0.0 \\
\hline \multicolumn{5}{|c|}{ Net accumulation of domestic arrears (ceiling on flow) ${ }^{3}$} \\
\hline Actual & 6.4 & -4.0 & 4.6 & -8.0 \\
\hline Program & -3.0 & -4.0 & -5.5 & -7.0 \\
\hline \multicolumn{5}{|l|}{ Indicative targets } \\
\hline Broad money (ceiling on stock) ${ }^{1}$ & & & & \\
\hline Actual & 363.6 & 371.3 & 399.1 & 393.9 \\
\hline Program & 365.9 & 389.5 & 394.3 & 422.3 \\
\hline Extended broad money (ceiling on stock) ${ }^{1}$ & & & & \\
\hline Actual & 431.2 & 444.1 & 477.1 & 473.7 \\
\hline Program & 425.5 & 453.7 & 459.3 & 491.1 \\
\hline Net Present Value of the Stock of Outstanding $D$ & & & & \\
\hline End-December 2007--Actual & & 240.9 & & 240.9 \\
\hline End-December 2008--Actual & & 331.5 & & 331.5 \\
\hline End-December 2009--Actual & & & & \\
\hline End-December 2007--Program & & 200.4 & & 200.4 \\
\hline End-December 2008--Program & & 286.4 & & 286.4 \\
\hline End-December 2009--Program & & 405.6 & & 405.6 \\
\hline Ceiling on stock of domestic debt ${ }^{9}$ & & & & \\
\hline Actual & 162.1 & 159.1 & 146.1 & 124.8 \\
\hline Program & 208.6 & 189.7 & 187.6 & 198.6 \\
\hline Memorandum items: & & & & \\
\hline General budget support (Millions of US $\$$ ) 4,10 & & & & \\
\hline Received & 104.2 & 219.8 & 255.3 & 312.5 \\
\hline Expected & 104.1 & 219.3 & 289.1 & 317.5 \\
\hline Of which: budget support grants (received) & 97.2 & 212.8 & 248.3 & 305.5 \\
\hline Of which: budget support grants (expected) & 97.1 & 212.3 & 282.1 & 310.5 \\
\hline Baseline privatization receipts (RF billion) & & & & \\
\hline Received & $\ldots$ & 3.6 & 3.6 & 3.6 \\
\hline Expected & & 3.5 & 3.5 & 17.1 \\
\hline
\end{tabular}

Sources: Rwandese authorities and IMF staff estimates and projections.

1 At the program exchange rate of RF545/US $\$$ for 2008.

2 Targets are calculated as an arithmetic average of the stock of reserve money on the last day of each calendar month in the quarter. Program projections are done quarterly.

${ }^{3}$ Numbers are cumulative from December $31,2007$.

${ }^{4}$ The domestic fiscal balance targets will be adjusted by the amount of grants exceeding or below programmed grants with the adjusters as specified in the TMU of February 12, 2008. Also the priority spending targets will be adjusted by the amount of grants exceeding or below programmed grants.

5 This is a continuous performance criterion. Excluded from the criterion are changes in indebtedness resulting from refinancing credits and rescheduling operations of existing debt, credits extended by the Fund, and US\$97.7 million in credit from the Exim Bank of India with concessionality of 40 percent for the construction of the hydro power plant at Nyabarongo.

6 Excludes arrears on obligations that are subject to rescheduling.

7 Ceiling on the stock of external debt (excluding normal imported-related credits) owed or guaranteed by the central governments, local government, or the NBR with original maturity of up to, and including one year.

${ }^{8}$ Figures indicate the NPV projections based on debt contracted at the test date.

${ }^{9}$ Numbers show end of period stocks. The stock of debt at end-2007 was revised from RF200.3 billion to RF 236 billion, which prompted revision of the program numbers for September and December 2008.

${ }^{10}$ Excluding external donor financing for demobilization and peacekeeping. 
Att. 1. Table 2. Rwanda: Quantitative Indicative Targets and Benchmarks for 2009-10 (Billions of Rwandan francs, unless otherwise indicated)

\begin{tabular}{|c|c|c|c|c|c|c|}
\hline \multirow{3}{*}{ Net foreign assets of the NBR (floor on stock) ${ }^{1}$} & \multicolumn{4}{|c|}{2009} & \multicolumn{2}{|c|}{2010} \\
\hline & \multirow[t]{2}{*}{ March } & \multirow[t]{2}{*}{ June } & \multirow[t]{2}{*}{ September } & \multirow[t]{2}{*}{ December } & \multirow[t]{2}{*}{ March } & \multirow[t]{2}{*}{ June } \\
\hline & & & & & & \\
\hline Actual (program exchange rate) & 267.0 & & & & & \\
\hline Adjusted program & 298.3 & & & & & \\
\hline Program & 311.6 & 298.0 & 341.6 & 326.1 & 325.0 & 301.8 \\
\hline \multicolumn{7}{|l|}{ Reserve money (ceiling on stock) ${ }^{2}$} \\
\hline Actual & 112.3 & & & & & \\
\hline Adjusted program & 114.8 & & & & & \\
\hline Program & 123.2 & 128.3 & 113.3 & 114.0 & 114.7 & 121.0 \\
\hline \multicolumn{7}{|l|}{ Net credit to the government (ceiling on flow) } \\
\hline Actual & 41.0 & & & & & \\
\hline Adjusted program & -2.3 & & & & & \\
\hline Program & -15.6 & -24.2 & -38.1 & -25.2 & -10.9 & 16.0 \\
\hline \multicolumn{7}{|l|}{ Domestic fiscal balance (floor on flow) } \\
\hline Actual & -47.3 & & & & & \\
\hline Adjusted program & -6.5 & & & & & \\
\hline Program & -35.8 & -72.2 & -145.8 & -209.3 & -25.7 & -61.4 \\
\hline \multicolumn{7}{|l|}{ Total priority spending (floor on flow) } \\
\hline Actual & 88.7 & & & & & \\
\hline Adjusted program & 63.6 & & & & & \\
\hline Program & 78.2 & 159.8 & 266.9 & 375.0 & 91.2 & 184.5 \\
\hline \multicolumn{7}{|l|}{ New nonconcessional external debt ${ }^{3}$} \\
\hline Actual & 0.0 & & & & & \\
\hline Program & 0.0 & 0.0 & 0.0 & 0.0 & 0.0 & 0.0 \\
\hline \multicolumn{7}{|l|}{ New external payment arrears (ceiling on stock) ${ }^{3,4}$} \\
\hline Actual & 0.0 & & & & & \\
\hline Program & 0.0 & 0.0 & 0.0 & 0.0 & 0.0 & 0.0 \\
\hline \multicolumn{7}{|l|}{ Short-term external debt (ceiling on stock) ${ }^{5}$} \\
\hline Actual & 0.0 & & & & & \\
\hline Program & 0.0 & 0.0 & 0.0 & 0.0 & 0.0 & 0.0 \\
\hline \multicolumn{7}{|l|}{ Net accumulation of domestic arrears (ceiling on flow) } \\
\hline Actual & 4.0 & & & & & \\
\hline Program & -1.6 & -3.3 & -5.4 & -8.4 & -1.6 & -3.3 \\
\hline \multicolumn{7}{|l|}{ Broad money (ceiling on stock) ${ }^{1}$} \\
\hline Actual & 431.7 & & & & & \\
\hline Program & 543.1 & 555.4 & 480.1 & 474.9 & 509.9 & 512.7 \\
\hline \multicolumn{7}{|l|}{ Actual } \\
\hline Net Present Value of the Stock of Outstanding Debt ( & & & & & & \\
\hline End-December 2007--Actual & & 240.9 & & 240.9 & & 240.9 \\
\hline End-December 2008--Actual & & 331.5 & & 331.5 & & 331.5 \\
\hline End-December 2009--Actual & & & & & & \\
\hline End-December 2007--Program & & 200.4 & & 200.4 & & 200.4 \\
\hline End-December 2008--Program & & 286.4 & & 286.4 & & 286.4 \\
\hline End-December 2009--Program & & 413.7 & & 413.7 & & 413.7 \\
\hline Ceiling on stock of domestic debt ${ }^{7}$ & & & & & & \\
\hline Actual & 98.8 & & & & & \\
\hline Program & 183.3 & 168.4 & 102.0 & 100.1 & 86.1 & 85.7 \\
\hline Memorandum items: & & & & & & \\
\hline Expected general budget support (Millions of US\$) ${ }^{8}$ & 96.2 & 173.7 & 330.1 & 424.7 & 57.8 & 72.0 \\
\hline Received & 19.5 & 159.6 & & & & \\
\hline Of which: budget support grants (received) & 19.5 & 159.6 & & & & \\
\hline Of which: budget support grants & 96.2 & 173.7 & 330.1 & 424.7 & 57.8 & 72.0 \\
\hline Baseline privatization receipts (RF billion) & 0.0 & 0.0 & 0.0 & 0.0 & 0.0 & 0.0 \\
\hline
\end{tabular}

Sources: Rwandese authorities and IMF staff estimates and projections.

${ }^{1}$ At the program exchange rate of RF555/US\$ through June 2009 and RF576.8 from September 2009 to June 2010.

${ }^{2}$ Targets are calculated as an arithmetic average of the stock of reserve money on the last day of each calendar month in the quarter.

${ }^{3}$ This is a continuous indicative target.

${ }^{4}$ Excludes arrears on obligations that are subject to rescheduling.

${ }^{5}$ Ceiling on the stock of external debt (excluding normal imported-related credits) owed or guaranteed by the central governments, local government, or the NBR with original maturity of up to, and including one year.

${ }^{6}$ Figures indicate the NPV projections based on debt contracted at the test date.

${ }^{7}$ Numbers show end of period stocks.

${ }^{8}$ Excluding external donor financing for demobilization and peacekeeping. 
INTERNATIONAL MONETARY FUND

RWANDA

Staff Report for the Sixth Review Under the Three-Year Arrangement Under the Poverty Reduction and Growth Facility and Request for Waivers of Nonobservance of Performance Criteria-Informational Annex

Prepared by the African Department

(In collaboration with other Departments)

Approved by Martine Guerguil and Dominique Desruelle

July 23, 2009

Contents

Page

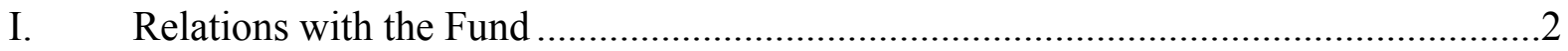

II. Joint Bank-Fund Work Program, August 2009-August 2010 .....................................

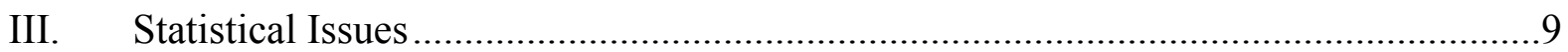




\section{APPENDix I. RWANDa: Relations With the Fund}

(As of June 30, 2009)

I. Membership Status: Joined: September 30, 1963;

$\underline{\text { Article VIII }}$

II. General Resources Account:

SDR Million

Quota

80.10

\%Quota

Fund holdings of currency

80.11

100.00

Reserve Position

0.00

100.02

Holdings Exchange Rate

III. SDR Department:

$\underline{\text { Net cumulative allocation }}$

Holdings

IV. Outstanding Purchases and Loans:

PRGF Arrangements
SDR Million

13.70

20.40

SDR Million

8.55
\%Allocation

100.00

148.94

\%Quota

10.68

\section{Latest Financial Arrangements:}

\begin{tabular}{lllrr} 
Type & \multicolumn{1}{c}{$\begin{array}{c}\text { Date of } \\
\text { Arrangement }\end{array}$} & $\begin{array}{c}\text { Expiration } \\
\text { Date }\end{array}$ & $\begin{array}{c}\text { Amount Approved } \\
\text { (SDR Million) }\end{array}$ & $\begin{array}{c}\text { Amount Drawn } \\
\text { (SDR Million) }\end{array}$ \\
PRGF & June 12, 2006 & August 14, 2009 & 8.01 & 6.84 \\
PRGF & August 12, 2002 & June 11, 2006 & 4.00 & 4.00 \\
PRGF & June 24, 1998 & April 30, 2002 & 71.40 & 61.88
\end{tabular}

\section{Projected Payments to Fund ${ }^{2}$}

(SDR Million; based on existing use of resources and present holdings of SDRs):

\begin{tabular}{lcccccc} 
& \multicolumn{6}{c}{ Forthcoming } \\
\cline { 2 - 6 } & $\underline{2009}$ & $\underline{2010}$ & $\underline{2011}$ & $\underline{2012}$ & $\underline{2013}$ \\
Principal & $\underline{0.02}$ & $\underline{0.04}$ & $\underline{0.04}$ & $\underline{0.04}$ & $\underline{0.04}$ \\
Charges/Interest & $\underline{0.02}$ & $\underline{0.10}$ & $\underline{0.44}$ & $\underline{0.72}$ & $\underline{1.18}$ \\
Total & & & &
\end{tabular}

\footnotetext{
${ }^{1}$ On June 4, the PRGF was extended from June 11, 2009 to August 14, 2009, to allow time for the completion of the sixth and final review, and for making the final disbursement under the PRGF arrangement.

${ }^{2}$ When a member has overdue financial obligations outstanding for more than three months, the amount of such arrears will be shown in this section.
} 
VII.

Implementation of HIPC Initiative:

I Commitment of HIPC assistance

Decision point date

Assistance committed by all creditors

(US\$ million) $^{11}$

Of which: IMF assistance (US\$ million)

(SDR equivalent in millions)

Completion point date

II Delivery of IMF assistance (SDR million)

Amount disbursed

Interim assistance

Completion point

Additional disbursement of interest income ${ }^{12}$

Total disbursements
Enhanced

framework

Dec 2000

695.50

63.40

46.79

Apr 2005

46.79

14.45

32.34

3.77

50.56

\section{Implementation of MDRI Assistance}

$\begin{array}{cc}\text { I Total Debt Relief (SDR Million) }^{13} & 52.74 \\ \text { Of which: MDRI } & 20.19 \\ \text { HIPIC } & 32.55\end{array}$

II Debt Relief by Facility (SDR million)

Delivery Date

January 2006

$\begin{array}{lll}\text { GRA } & \text { PRGF } & \text { Total } \\ \text { NA } & 52.74 & 52.74\end{array}$

\footnotetext{
${ }^{11}$ Assistance committed under the original framework is expressed in net present value (NPV) terms at the completion point, and assistance committed under the enhanced framework is expressed in NPV terms at the decision point. Hence these two amounts can not be added.

${ }^{12}$ Under the enhanced framework, an additional disbursement is made at the completion point corresponding to interest income earned on the amount committed at the decision point but not disbursed during the interim period.

${ }^{13}$ The Multilateral Debt Relief Initiative (MDRI) provides 100 percent debt relief to eligible member countries that are qualified for the assistance. The debt relief covers the full stock of debt owed to the Fund as of end2004 which remains outstanding at the time the member qualifies for such debt relief. The MDRI is financed by bilateral contributions and the Fund's own resources, as well as the resources already disbursed to the member under the HIPC Initiative (see Section VII above).
} 
Decision point-point at which the IMF and the World Bank determine whether a country qualifies for assistance under the HIPC Initiative and decide on the amount of assistance to be committed.

Interim assistance - amount disbursed to a country during the period between decision and completion points, up to 20 percent annually and 60 percent in total of the assistance committed at the decision point (or 25 percent and 75 percent, respectively, in exceptional circumstances).

Completion point - point at which a country receives the remaining balance of its assistance committed at the decision point, together with an additional disbursement of interest income as defined in footnote 3 above. The timing of the completion point is linked to the implementation of pre-agreed key structural reforms (i.e., floating completion point).

\section{Safeguards Assessments:}

Under the Fund's safeguards assessment policy, the National Bank of Rwanda (NBR) was subject to a safeguards assessment with respect of the PRGF arrangement approved on June 12, 2006. The update assessment proposed recommendations to address continuing vulnerabilities in the external audit and financial reporting areas. The implementation of these measures is being monitored by IMF staff.

\section{Exchange System:}

The currency of Rwanda is the Rwandan franc. Under the revised classification of the exchange rate arrangements, Rwanda has "other managed arrangement". The official reference exchange rate is determined daily by the National Bank of Rwanda using a moving average of commercial banks and NBR transactions. From January 2009, the averaging period has been extended from 5 to 10 days. The NBR buys and sells foreign exchange from commercial banks on demand at the rates that differ from official rate by a certain margin.

On December 1998, Rwanda accepted the obligations under Article VIII, Sections 2, 3 and 4 of the IMF and maintains a system free of restrictions on the making of payments and transfers for current international transactions.

\section{Article IV Consultation:}

Rwanda is on the revised 24-month consultation cycle. The Executive Board discussed the staff report for the 2008 Article IV consultation (Country Report No. 09/58) on January 12, 2009. 


\section{FSAP Participation, ROSCs, and OFC Assessments:}

A Report on Observance of Standards and Codes on Fiscal Transparency (ROSC) was issued in July 2003. A Financial Sector Assessment Program (FSAP) took place in February 2005. Rwanda has not had an Offshore Financial Center (OFC) assessment.

\section{Technical Assistance:}

2004 AFRITAC East expert on financial regulations.

2004 AFRITAC East mission on treasury reforms.

2004 AFRITAC East expert on implementing cash flow planning and banking arrangements.

2004 FAD tax administration expert on strengthening of revenue administration.

2004 FAD mission on revenue administration.

2004 MFD/LEG mission on Anti-Money Laundering and Combating the Financing of Terrorism.

2004 MFD expert on banking supervision and regulation.

2004 MFD expert on monetary policy, monetary operations, and money markets.

2004 MFD missions on on-site banking supervision.

2005 AFRITAC East expert follow up on cash management.

2005 MFD/LEG mission on Anti-Money Laundering and Combating the Financing of Terrorism.

2005 MFD mission on financial statements of specific bank.

2005 MFD-WB joint FSAP mission.

2005 LEG mission on customs legislation.

2005 STA mission on Balance of Payments statistics.

2005 LEG mission on tax legislation.

2005 FAD mission on public accounting: decentralized accounting for central government.

2005 FAD expert on tax administration.

2005 MFD resident expert on monetary operations, monetary policy, money markets.

2005 MFD expert on banking supervision.

2005 LEG mission on drafting an amendment to the banking law.

2005 MFD mission on banking supervision and bank restructuring.

2006 AFRITAC East experts on establishing intergovernmental fiscal unit in the MOF.

2006 FAD advisor trade facilitation.

2006 MFD FSAP-follow up advisory mission on monetary and foreign exchange operations and NBR internal audit.

2006 LEG mission to strengthen legal and regulatory framework for bank supervision.

2006 AFRITAC East advisors on finalization of OBL and financial regulations

2007 AFRITAC East advisors on workshops for implementation of OBL and financial regulations.

2007 MCM expert on bank restructuring. 
2007 MCM expert on foreign exchange operations.

2008 LEG mission to assist the Ministry of Finance in modernizing the tax system.

2008 LEG mission on banking legislation.

2008 MCM expert on foreign exchange operations.

2008 MCM mission to assist in building up internal audit in the central bank.

2008 FAD expert on revenue administration.

2008 AFRITAC East advisors on (i) improving the budget preparation process and budget accounting, and (ii) changing to the GFS2001- based economic classification (jointly with STA).

2008 MCM mission on monetary policy operations, foreign exchange operations, and assessment of the financial health of banks.

2008 AFRITAC East advisors on enhancement of national accounts estimation.

2009 MCM mission on liquidity management.

2009 MCM mission on bank supervision.

2009 STA mission on rebasing consumer and producer price indices.

2009 FAD/AFRITAC East mission to review the progress and set the program for TA in budget reform and PFM.

2009 FAD/AFRITAC East mission on customs risk management and valuation.

2009 AFRITAC East advisors on (i) enhancing comprehensiveness and transparency of the fiscal budget and (ii) reform strategy for introduction of program budgeting.

2009 AFRITAC East advisors on quarterly GDP compilation.

2009 AFRITAC East workshop on national accounts.

2009 AFRITAC East advisors on (i) developing a framework for adopting a capital charge for market risk, (ii) enhancements to offsite surveillance system, and (iii) payment system modernization.

2009 AFRITAC East advisors on operational policy development and compliance management in revenue administration.

2009 AFRITAC East workshop on strengthening tax audit and collection enforcement.

\section{Resident Representative:}

Mr. Dmitry Gershenson assumed his duties as Resident Representative in February 2009.

\section{Management Visit:}

The Deputy Managing Director, Mr. Portugal, visited Rwanda during May 3-5, 2007. 


\section{APPENDIX II. RWANDA: JOINT BANK-FUND WORK PROGRAM}

VIII. AuguSt 2009 - August 2010

\begin{tabular}{|c|c|c|c|}
\hline Title & Products /Activity & $\begin{array}{l}\text { Provisional } \\
\text { timing of mission } \\
\text { (if relevant) }\end{array}$ & $\begin{array}{l}\text { Expected } \\
\text { delivery date }\end{array}$ \\
\hline \multicolumn{4}{|c|}{ I. Mutual Information on Relevant Work Program } \\
\hline \multirow{14}{*}{$\begin{array}{l}\text { Bank } \\
\text { Work } \\
\text { Program }\end{array}$} & \multicolumn{3}{|c|}{ A. Strategy and Analytical Work } \\
\hline & Education - Country Status Report & & Ongoing \\
\hline & $\begin{array}{l}\text { First Rwanda: Financial Sector } \\
\text { Development Program (FSDP) II }\end{array}$ & & March 2010 \\
\hline & $\begin{array}{l}\text { Technical Assistance for Public } \\
\text { Expenditure Management in Rwanda }\end{array}$ & & June 2010 \\
\hline & Finance and Private Sector Dialogue & & Ongoing \\
\hline & \multicolumn{3}{|c|}{ B. Ongoing and New Projects } \\
\hline & Urgent Electricity Rehabilitation & & July 2009 \\
\hline & Support to Agricultural Policy Reforms & & Ongoing \\
\hline & Decentralization and Community Developr & ent & June 2010 \\
\hline & Public Sector Capacity Building Project & & Ongoing \\
\hline & E-Rwanda & & Ongoing \\
\hline & Regional Trade Facilitation & & Ongoing \\
\hline & Transport Sector Development & & Ongoing \\
\hline & Competitiveness and Enterprise Project & & Ongoing \\
\hline \multirow[t]{7}{*}{$\begin{array}{l}\text { IMF Work } \\
\text { Program }\end{array}$} & \multicolumn{3}{|c|}{ A. Missions } \\
\hline & Staff visit & October 2009 & November 2009 \\
\hline & Discussions on a new program & $\begin{array}{l}\text { February/March } \\
2010\end{array}$ & May 2010 \\
\hline & \multicolumn{3}{|c|}{ B. Analytical Work } \\
\hline & $\begin{array}{l}\text { Study on macroeconomic convergence } \\
\text { among the EAC countries }\end{array}$ & & March 2010 \\
\hline & $\begin{array}{l}\text { Spillovers from the world economic crisis } \\
\text { to the EAC countries }\end{array}$ & & April 2010 \\
\hline & Foreign exchange reserve adequacy in & & January 2010 \\
\hline
\end{tabular}




\begin{tabular}{|c|c|c|c|}
\hline Title & Products /Activity & $\begin{array}{l}\text { Provisional } \\
\text { timing of mission } \\
\text { (if relevant) }\end{array}$ & $\begin{array}{l}\text { Expected } \\
\text { delivery date }\end{array}$ \\
\hline & the EAC countries & & \\
\hline \multicolumn{4}{|c|}{ C. Technical Assistance } \\
\hline \multicolumn{4}{|c|}{$\begin{array}{l}\text { Fiscal: Tax and customs administration; } \\
\text { revenue forecasting; PFM }\end{array}$} \\
\hline \multicolumn{4}{|c|}{$\begin{array}{l}\text { Monetary: Monetary policy operations; } \\
\text { bank liquidity; interbank markets in local } \\
\text { currency and foreign exchange }\end{array}$} \\
\hline & \multicolumn{3}{|l|}{$\begin{array}{l}\text { Financial: Payment systems; bank } \\
\text { supervision }\end{array}$} \\
\hline & $\begin{array}{l}\text { Statistics: National accounts; balance of } \\
\text { payments; government Finance }\end{array}$ & & \\
\hline
\end{tabular}

\section{Request for Work Program Inputs}

\begin{tabular}{lll}
\hline $\begin{array}{l}\text { Bank } \\
\text { Request } \\
\text { to Fund }\end{array}$ & $\begin{array}{l}\text { Impact of the global economic crisis on } \\
\text { key macro indicators and growth in } \\
\text { Rwanda } \\
\text { Sharing macro-framework updates }\end{array}$ & Ongoing \\
\hline $\begin{array}{l}\text { Fund } \\
\text { Request } \\
\text { to Bank }\end{array}$ & $\begin{array}{l}\text { Assessment of key infrastructure projects } \\
\text { undertaken by the government. }\end{array}$ & As needed \\
\hline & $\begin{array}{l}\text { Assessment of the PFM and public } \\
\text { service reform program. }\end{array}$ & As needed \\
\hline
\end{tabular}

\section{Agreement on Joint Products}

\begin{tabular}{lll}
\hline Joint & Public and External Debt Sustainability & May 2010 \\
products & $\begin{array}{l}\text { Analysis. } \\
\text { Collaborate together with other } \\
\text { development partners on the annual } \\
\text { review of general budget support. }\end{array}$ & March 2010 \\
& $\begin{array}{l}\text { Collaborate on PFM reform program. } \\
\text { Collaborate on financial sector reform } \\
\text { program. }\end{array}$ & Ongoing \\
& Ongoing \\
\hline
\end{tabular}


Press Release No. 09/276

International Monetary Fund

FOR IMMEDIATE RELEASE

Washington, D.C. 20431 USA

July 31, 2009

\section{IMF Executive Board Completes the Sixth Review of Rwanda's PRGF Arrangement and Approves US\$1.8 Million Disbursement}

The Executive Board of the International Monetary Fund (IMF) completed today the sixth review under the three-year Poverty Reduction and Growth Facility (PRGF) arrangement with Rwanda (see Press release No. 06/121). The completion of the review enables the immediate disbursement of SDR 1.17 million (about US\$ 1.8 million), bringing the total amount disbursed under the program to SDR 8.01 million (US\$12.4 million).

The Board also approved the authorities' request for four waivers regarding nonobservance of performance criteria related to guaranteeing of new non-concessional external debt with original maturity of more than one year, and the modification of multiple currency practices.

Following the Executive Board discussion, Mr. Murilo Portugal, Deputy Managing Director and Acting Chairman, stated:

"The Rwandan authorities are to be commended for the satisfactory implementation of their program supported by the PRGF arrangement, notwithstanding an increasingly difficult external environment. Macroeconomic stability has strengthened, and important strides were made in establishing the base for sustained growth and further poverty reduction. Since early 2009, the global crisis has resulted in a fall in export revenue and a slowdown in economic activity. The policy responses to these challenges have been broadly appropriate, including increased fiscal spending and an easing of the monetary policy stance. For the 2009/10 fiscal year, the main challenge will be to cushion the impact of the global economic crisis while preserving fiscal and external sustainability.

"The fiscal program envisages a moderate stimulus to minimize the adverse effects of the economic slowdown. The higher budget deficit will be financed by withdrawing government deposits at the central bank. Gradual fiscal consolidation over the medium term should focus on increasing public revenues while safeguarding priority spending. 
"The monetary program will aim to balance the objectives of achieving single digit inflation while providing sufficient liquidity to the banking system. A limited transfer of government deposits from the central bank to commercial banks will help sustain long-term credit in the second half of 2009. Looking ahead, a more proactive use of available monetary policy instruments, including interest rate and exchange rate flexibility, would strengthen the effectiveness of monetary policy and support the long-term sustainability of the balance of payments.

"The authorities' structural reform agenda is expected to further improve public services delivery. The planned reforms in public financial management and revenue administration are encouraging. Implementation of the financial sector reform program, including strengthened supervision and improved risk management by the banks, will support financial deepening." 


\section{Statement by Laurean Rutayisire, Executive Director for Rwanda July 31, 2009}

My Rwandan authorities would like to thank staff for the constructive discussions held in Kigali during their recent missions in connection with the sixth review of the PRGF arrangement. My authorities would also like to thank the Executive Board and Management for the continued support to Rwanda in the pursuit of its PRGF program and broader development goals.

Rwanda is at an important juncture in its relationship with the Fund and the donor community. After successive PRGF-supported programs, albeit the daunting challenges that still lie ahead, Rwanda has strengthened macroeconomic stability and made important strides in establishing the base for sustained growth and further poverty reduction. My authorities have all through the course of their PRGF program demonstrated ownership as evidenced by the commendable performance recorded. Amid the global economic slowdown, real output grew by 11.2 percent in 2008 , inflation was subdued, and the country has continued to foster structural change in key sectors of the economy. In light of these achievements, my Rwandan authorities would like to request the support of the Board for the completion of the sixth review under the PRGF arrangement and waivers of nonobservance of performance criteria.

\section{Recent Economic Developments and Achievements under the PRGF}

Owing to my authorities' determined implementation of sound policies, Rwanda continues to post significant output growth and poverty reduction. For the first time over the last five years, real GDP grew by double-digit, reaching 11.2 percent in 2008 despite the global slowdown. The main drivers of this performance were the robust expansion in agriculture, manufacturing, services and construction sectors. Inflation that accelerated to above 20 percent in the second half of 2008 as a result of high world commodity prices declined sharply to 12.7 percent in May 2009, and my authorities are committed to bringing it down to single digit.

Rwanda was largely unaffected by the current global economic crisis until early 2009. In 2008 , the country recorded buoyant receipts from traditional exports, coffee and tea, and mining. External resources, notably foreign direct investment (FDI), donor aid, and concessional public borrowing were also flowing in at a steady pace. Despite rising imports related to the strong economic growth, the current account deficit was comfortably covered and at year-end 2008, Rwanda accumulated international reserves to over 5 months of imports. As a consequence of the crisis, the first months of 2009 witnessed a reversal of this momentum, with a sharp decline in exports receipts and domestic activity, particularly in the construction, mining, and tourism sectors.

Notwithstanding the negative spillovers from the world economic crisis, my Rwandan authorities' recent performance under the PRGF has been satisfactory. All quantitative performance criteria for the sixth review were met. The underperformance on some targets was either temporary or due to factors out of the authorities' control. In the fiscal area, my 
authorities posted higher revenues than envisaged under the program, amid the impact of the global crisis. Moreover, the cautious approach used by my authorities to save and accumulate deposits up to 1.8 percent of GDP in 2008, provided a buffer to the government to make up the delayed donor disbursements in the first quarter of 2009. On the expenditure side, my authorities have emphasized spending on capital projects, thus keeping up their overall goal of strengthening the infrastructure base of the economy. In addition, key spending on priority sectors was safeguarded. In the monetary sector, my authorities have acted promptly to address the liquidity shortages that arose in early 2009. By repaying a substantial amount of T-bills and reducing the reserve requirement from 8 percent to 5 percent, large amounts of liquidity (up to 1.5 percent of GDP) were injected into the banking system. My authorities intend to step up actions to further remove structural impediments to deposits in the banking system.

The PRGF-supported program has also helped my authorities to garner donor support while keeping pace with debt sustainability. As a post-HIPC and post-MDRI country, Rwanda had to strike the right balance between mobilizing the financial resources for its development needs without building up new unsustainable public debt. In that regard, the ending lowaccess PRGF helped mobilize highly concessional assistance from donors, bringing Rwanda's rating of debt distress risk from high to moderate in late 2008.

\section{The Way Forward}

Building on the commendable results achieved under the PRGF, my Rwandan authorities have laid out their policies for the fiscal year 2009/10 and for the medium term as well. These policies especially address three main factors that will influence the macroeconomic outlook for the period ahead, namely: (i) the return of agriculture to normal growth trend; (ii) the impact of the global economic crisis; and (iii) the effect of liquidity shortages on the economy. To achieve the growth rate for 2009 projected at 5.3 percent, my authorities intend to intensify investment and extension service activities in agriculture as the lead sector.

\section{Fiscal policy and debt management}

My authorities' fiscal policy will encounter an important milestone beginning this year as Rwanda aligns itself to the EAC budget calendar and starts to implement the EAC Common External Tariff from 1st July 2009 under the Customs Management Act. To make up for the revenue loss resulting from this change and from the slowing economic activity, my authorities envisage a number of measures including: (i) additional revenue measures of about 0.4 percent of GDP; and (ii) a stimulus by withdrawing government deposits at the NBR up to 1 percent of GDP. Total spending is set to decline compared to 2008, by about 1 percent of GDP, while safeguarding priority spending on priority areas notably, agriculture, energy, roads, ICT, education and health, water and sanitation.

Over the medium term, my authorities are committed to preserve fiscal and external sustainability. Revenue is projected to recover from the effects of the current slowdown and increase by at least 0.2 percent of GDP per year, reflecting also greater efficiency of tax 
collection. Based on current grants pledges, donor support is projected to decline by 2 to 3 percent compared to its 2009/10 level. To fill the financial gap, my authorities are cognizant of the need to step up efforts to strengthen domestic revenue and contain expenditure growth. A gradual contraction, reflecting the reduction in externally financed capital projects, should edge down expenditure from 26.9 percent of GDP in 2009/10 to 25.2 percent in 2010/12. Should the fiscal measures not suffice to meet the government financial needs, my authorities are committed to recurring to external borrowing only on concessional terms. As for the recent contracting of new nonconcessional external debt from China ExImBank, my authorities have liaised with developments partners and put forward factors such as the project's viability, confirmed by the World Bank and the minor impact on the overall debt outlook.

\section{Monetary and exchange rate policy}

My authorities' monetary program will be geared toward maintaining inflation to single digits while providing sufficient liquidity to the banking system to support private activity. To address the inflation issue, reserve money will remain the anchor of the monetary program of my authorities for the fiscal year 2009/10. In that regard, the limit on reserve money growth will be set at about 6.5 percent and 7.5 percent respectively for the JulyDecember 2009 and January-June 2010 periods. To face an unexpected deterioration of the balance of payments stemming from the global crisis, the authorities will allow greater flexibility in the exchange rate.

As regards the liquidity issue, my authorities have decided to temporarily move part of government deposits at the NBR to commercial banks that increase the stock of their investment lending with maturity of over 3 years. This measure will help increase liquidity and boost private lending in the short run. Furthermore, my authorities will take actions to improve the functioning of the banking system and bank supervision. First, they will finalize and implement a new liquidity regulation, which will require banks to comprehensively report on maturity of assets and liabilities, and on this basis, a new and more comprehensive liquidity ratio will be developed. Other initiatives include: (i) implementation of the recently approved regulation on classification of nonperforming loans and provisioning;

(ii) strengthening of risk-based bank supervision and augmentation of the supervisory capacity of the NBR; and (iii) enforcement of better risk management practices and corporate governance in the commercial banks.

\section{Structural policies}

For the fiscal year 2009/10 and the medium term, my Rwandan authorities' structural policies will focus on enhancing the productivity of the agricultural and export sectors, improving conditions for the private sector development and strengthening the effectiveness of public financial management. On export promotion, a National Export Board will be established soon to deal with all agricultural exports while the strategy will continue to focus on enhancing the productivity in traditional sectors and diversifying the export base. The Financial Sector Development Plan is expected to be fully implemented by June 2010. It 
encompasses key goals such as improving access to credit, raising the gross national savings from 8.5 percent of GDP in 2008/09 to 10 percent by 2012, improving the pension sector, and completing a regulation and legal framework for the insurance industry.

My authorities attach a particular importance to the improvement of the business climate with the view to lowering the cost of doing business. A key step in this endeavor will be the modernization of Rwanda's business registration. As well, online operations will be further developed, and it is planned to make it possible to register a business on-line by the end of 2009. My authorities also intend to develop a Public Private Partnership framework to foster private sector participation in infrastructure provision, while enhancing the government's capacity to manage related fiscal risks. Consistent with their fiscal sustainability goal, the authorities will continue to further structural change in the PFM and the whole fiscal sector. Tax reforms will accommodate and enhance the effectiveness of the decentralization process. Measures will be taken to enhance taxpayer compliance and increase efficiency of operations and minimize transaction costs by decentralizing tax services away from headquarters and closer to businesses.

\section{Conclusion}

Under the framework of the PRGF arrangement, my Rwandan authorities have continued to achieve good results towards their overall goal to deliver better living standards to populations. Beside the improved domestic policymaking, the assistance of the Fund and the donor community has been instrumental in posting these results. Furthermore, the structural change taking hold and improvements on several fronts such as accountability and governance have contributed to strengthen Rwanda's stature in the international community.

Going forward, it is my authorities' strong belief that the achievements in macroeconomic stability and structural reforms can be consolidated by further program relationship with the Fund. My authorities' leaning is towards a Policy support Instrument which they view as the best-suited framework for future cooperation with the Fund. 